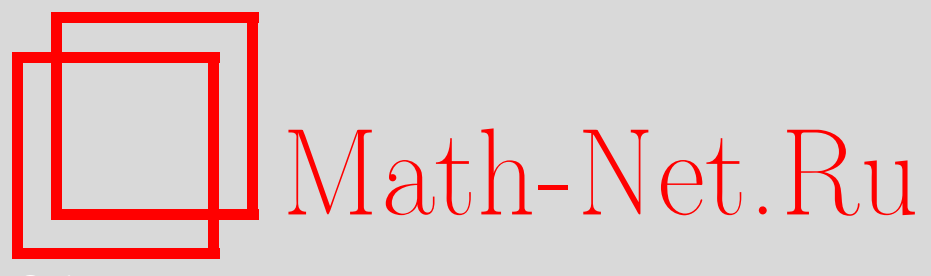

В. Н. Дубинин, Методы геометрической теории функций в классических и современных задачах для полиномов, УМН, 2012, том 67, выпуск 4, 3-88

DOI: https://doi.org/10.4213/rm9488

Использование Общероссийского математического портала Math-Net.Ru подразумевает, что вы прочитали и согласны с пользовательским соглашением http://www . mathnet.ru/rus/agreement

Параметры загрузки:

IP : 54.164 .48 .24

26 апреля 2023 г., $12: 32: 33$

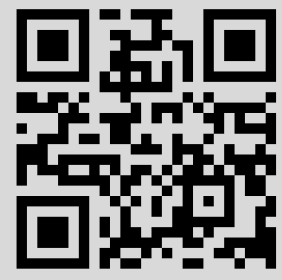




\section{Методы геометрической теории функций в классических и современных задачах для полиномов}

\section{В. Н. Дубинин}

Статья представляет собой обзор классических и современных теорем для полиномов, доказанных методами геометрической теории функций. Основное содержание статьи составляют результаты автора и его учеников, установленные с помощью принципов мажорации для голоморфных функций, теории однолистных функций, теории емкостей и симметризации. Приводятся вспомогательные утверждения и доказательства некоторых теорем.

Библиография: 124 названия.

Ключевые слова: принципы мажорации, лемма Шварца, однолистные функции, емкости, симметризация, неравенства, полиномы, критические точки, критические значения, рациональные функции.

\section{СОДЕРЖАНИЕ}

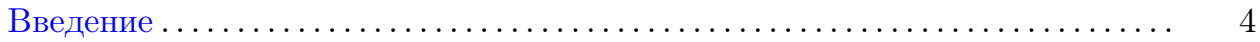

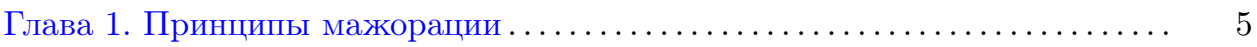

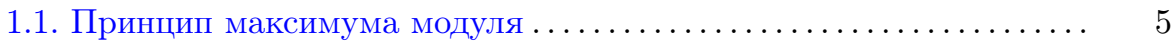

1.2. Обобщения и дополнения леммы Шварца ................. 11

1.3. Применение леммы Шварца к неравенствам для полиномов .... 13

1.4. О целых функциях экспоненциального типа............... 18

1.5. Дополнение к принципу Линделёфа с приложениями к полино-

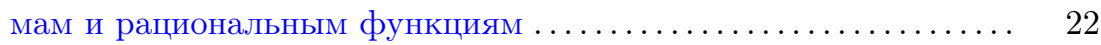

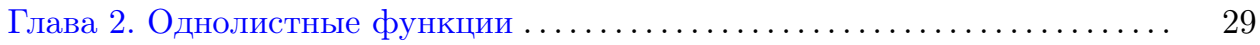

2.1. Построение однолистных функций...................... 29

2.2. Теоремы покрытия для полиномов................... 32

2.3. Неравенства с участием производных................. 37

2.4. Неравенства для коэффициентов .................... 44

2.5. Полиномы с критическими значениями на отрезке .......... 48

2.6. О полиномах с криволинейной мажорантой .............. 52

Работа выполнена при поддержке РФФИ (грант № 11-01-00038) и ДВО РАН (грант № $12-\mathrm{I}-\mathrm{OMH}-02)$.

(C) В. Н. Дувинин, 2012 
Глава 3. Емкости конденсаторов и симметризация ....................

3.1. Обобщенные конденсаторы и асимптотика их емкости при вы-

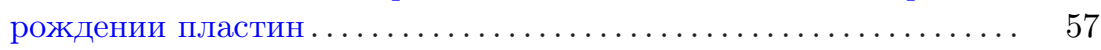

3.2. Простейшие применения конформной емкости ............. 60

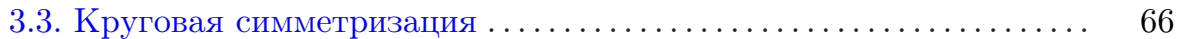

3.4. K теоремам о конечном приращении................. 67

3.5. О свойствах полиномов внутри лемнискаты ............. 70

3.6. Неравенства для критических значений полиномов ........... 74

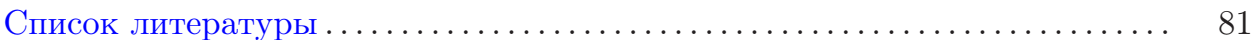

\section{Введение}

Неравенства для полиномов и их производных имеют богатую историю и многочисленные приложения (см., например, [1]-[6]). Вместе с тем применение методов геометрической теории функций к получению этих неравенств (для простейших аналитических функций) осуществляется в меньшей степени, чем можно было бы ожидать. В серии работ автора и его учеников была сделана попытка частично устранить указанный пробел [7]-[36]. Цель данной статьи дать краткий обзор полученных при этом результатов и показать на примерах простых доказательств классических и современных теорем о полиномах суть новых подходов по применению некоторых методов теории функций комплексного переменного. Всюду ниже приняты следующие обозначения:

$$
P(z)=c_{0}+c_{1} z+\cdots+c_{n} z^{n}, \quad c_{n} \neq 0, \quad n \geqslant 1,
$$

- алгебраический полином степени $n$ с комплексными коэффициентами $c_{k}, k=$ $0,1, \ldots, n$

$$
\begin{aligned}
L(P) & =\min \{\operatorname{Re} P(z):|z|=1\}, & & H(P)=\max \{\operatorname{Re} P(z):|z|=1\}, \\
m(P) & =\min \{|P(z)|:|z|=1\}, & & M(P)=\max \{|P(z)|:|z|=1\} ;
\end{aligned}
$$

величины $\bar{L}(P), \bar{H}(P), \bar{m}(P)$ и $\bar{M}(P)$ определяются так же, как соответствующие величины выше, но с заменой окружности $|z|=1$ на отрезок $[-1,1]$. Многие результаты данной статьи состоят в оценках для полиномов $P$ в зависимости от выписанных выше величин. Обсуждаются также неравенства для критических точек и критических значений полиномов $P$. В роли экстремальных полиномов выступают, как правило, полиномы вида $c_{0}+c_{n} z^{n}, c_{1} z+c_{n} z^{n}$ при некоторых $c_{0}, c_{1}, c_{n}$ либо полиномы вида $a T_{n}(z)+b$, где $a$ и $b$ - постоянные, a $T_{n}(z)$ означает, как обычно, полином Чебышёва первого рода. Напомним, что в терминах конформных отображений полином $T_{n}(z)=2^{n-1} z^{n}+\cdots$ можно определить как суперпозицию обратной функции ЖКуковского, степенной функции и функции Жуковского:

$$
T_{n}(z)=\frac{1}{2}\left(\left(z+\sqrt{z^{2}-1}\right)^{n}+\left(z-\sqrt{z^{2}-1}\right)^{n}\right), \quad z \in \mathbb{C} .
$$

Материал статьи группируется по методам геометрической теории функций, применение которых раскрывается в процессе решения типичных задач для полиномов. В случае сложных доказательств мы приводим лишь результаты и некоторые комментарии к ним. В первой главе приводятся новые применения 
классической леммы Шварца и ее современных версий. Кроме того, мы дополняем ее обобщение - принцип Линделёфа и даем приложения этого принципа не только к полиномам, но и к рациональным функциям с предписанными полюсами, а также к некоторым целым функциям экспоненциального типа. Во второй главе развивается методика построения по заданному алгебраическому полиному конформного и однолистного отображения некоторого класса с последующим применением теории однолистных функций. Ограничиваясь лишь небольшим числом утверждений этой теории, мы надеемся показать эффективность предлагаемого подхода к неравенствам для полиномов. Наконец, в третьей главе собраны результаты, полученные методами теории потенциала и симметризации [37], [38]. Для удобства чтения введены разделы 3.1 и 3.3, которые носят вспомогательный характер. С применением симметризации связаны особые надежды, так как экстремальные полиномы в известных открытых гипотезах о полиномах обладают той или иной симметрией.

Автор не претендует на полноту изложения затронутых здесь вопросов. Основное содержание статьи обсуждалось в разное время на семинарах А. А. Гончара в МИ РАН, Г. В. Кузьминой в ПОМИ РАН и О. Мартио и М. Вуоринена в Хельсинкском университете (Финляндия), а также доказывалось с разной степенью полноты на международных конференциях в городах Халле (Германия, 2002), Макао (Китай, 2010) и Вюрцбурге (Германия, 2010).

\section{Глава 1. Принципы мажорации}

Принципы мажорации и их приложения играют существенную роль в геометрической теории функций комплексного переменного (см. [39; гл. VIII]). В настоящей главе рассматриваются простые применения принципа максимума модуля, классических и современных версий леммы Шварца, а также дополнения к принципу Линделёфа. Указанные принципы содержат довольно общие неравенства для полиномов и рациональных функций с предписанными полюсами. Успех в применении этих принципов зависит от удачного построения аналитических функций, к которым они применяются.

1.1. Принцип максимума модуля. Хорошо известно, что если функция $f$ голоморфна и отлична от постоянной в данной области комплексной плоскости, то ее модуль не может достигать локального максимума в какой-либо точке этой области. Удивительно, что этот простой факт теории аналитических функций не использовался в должной мере при доказательстве классических неравенств для алгебраических полиномов. Рассмотрим применение принципа максимума модуля на примере следующего результата К. Дочева [40].

Теорема 1.1. Если полином $P(z)=c_{0}+\cdots+c_{n} z^{n}, c_{n} \neq 0$, с вещественньми коэффициентами $c_{k}, k=0,1, \ldots, n$, нормирован условием $\bar{M}(P)=1$, то для любого $\rho>1$ образ эллипса $|z-1|+|z+1|=\rho+1 / \rho$ при отображении $w=P(z)$ лежст внутри эллипса $|w-1|+|w+1|=\rho^{n}+1 / \rho^{n}$. Здесь экстремальным является случай, когда $P(z)$ совпадает с полиномом Чебъшёва первого рода $T_{n}(z)$, который $n$-кратно покрывает второй эллипс первым.

ДокАзАТЕЛЬСтво. Обозначим через $\eta=\Phi(w)$ функцию, конформно и однолистно отображающую внешность отрезка $[-1,1]$ на круг $|\eta|<1$ так, что 
$\Phi(\infty)=0$ и $\Phi(1)=1$. По принципу максимума модуля аналитической функции, примененному в каждой связной компоненте открытого множества

$$
\left\{\zeta:|\zeta|<1, P\left(\frac{\zeta+1 / \zeta}{2}\right) \notin[-1,1]\right\},
$$

заключаем, что модуль функции

$$
\zeta^{n}\left[\Phi\left(P\left(\frac{\zeta+1 / \zeta}{2}\right)\right)\right]^{-1}
$$

не превосходит на этом множестве единицы. Используя представление полинома Чебышёва

$$
T_{n}(z)=\frac{1}{2}\left[\Phi^{n}(z)+\Phi^{-n}(z)\right]
$$

получаем неравенство

$$
\left|\Phi\left(P\left(\frac{\zeta+1 / \zeta}{2}\right)\right)\right| \geqslant\left|\zeta^{n}\right|=\left|\Phi\left(T_{n}\left(\frac{\zeta+1 / \zeta}{2}\right)\right)\right|,
$$

которое равносильно утверждению теоремы 1.1. Теорема доказана.

К. Дочев получил указанный результат путем оригинального исследования соответствующих тригонометрических полиномов. В работе [8] приводится усиление этого геометрического факта с учетом старшего коэффициента $c_{n}$. Теорема 1.1 содержит ранний результат Бернштейна, состоящий в том, что для любой точки $z$ внутри эллипса $|z-1|+|z+1|=\rho+1 / \rho$ выполняется неравенство

$$
|P(z)| \leqslant \rho^{n}
$$

(см. [1; с. 448]). Кроме того, эта теорема содержит точную оценку Р. Дуффина и А. Шеффера [41]:

$$
|P(z)| \leqslant \frac{1}{2}\left(\rho^{n}+\frac{1}{\rho^{n}}\right),
$$

справедливую для тех же точек $z$. При достаточно больших значениях $\rho$ теорема 1.1 дает оценку

$$
\left|c_{n}\right| \leqslant 2^{n-1}
$$

которая равносильна классическому свойству монома Чебышёва $2^{1-n} T_{n}$ наименее уклоняться от нуля на отрезке $[-1,1]$. Непосредственно из теоремы 1.1 вытекает неравенство Чебышёва

$$
|P(x)| \leqslant\left|T_{n}(x)\right| \bar{M}(P),
$$

справедливое для любых вещественных полиномов $P$ степени $n$ и любых $x \in$ $\mathbb{R} \backslash[-1,1]$ (см. [2; с. 235]). Устремляя параметр $\rho$ в теореме 1.1 к единице, приходим к неравенству Бернштейна-Сегё

$$
\left|P^{\prime}(x) \sqrt{1-x^{2}}\right| \leqslant n \sqrt{1-P^{2}(x)}, \quad x \in[-1,1],
$$

содержащему классическое неравенство Бернштейна

$$
\left|P^{\prime}(x)\right| \leqslant \frac{n}{\sqrt{1-x^{2}}}, \quad x \in[-1,1]
$$


(см., например, [2; с. 233]). Можно показать, что теорема 1.1 содержит также неравенство Маркова

$$
\left|P^{\prime}(x)\right| \leqslant n^{2}, \quad x \in[-1,1]
$$

(см. [8; лемма 4.1]).

В качестве другого использования принципа максимума модуля приведем доказательство следующей теоремы искажения для полиномов на окружности.

Теорема 1.2. Для любого полинома $P$ степени $n$ и любой точки $z$, лежащей на окружности $|z|=1$, и такой, что $P(z) \neq 0$, справедливо неравенство

$$
\left|\operatorname{Im} \frac{z P^{\prime}(z)}{P(z)}\right| \leqslant \frac{n}{2} \sqrt{\frac{M^{2}(P)}{|P(z)|^{2}}-1} .
$$

Равенство в (1.1) достигается в случае $P(z)=c_{0}+c_{n} z^{n}$ для любых точек $z$ таких, что $|z|=1, z^{n} \neq-c_{0} / c_{n}$, где $c_{0} u c_{n}$ - произвольные комплексные числа, удовлетворяющие условию $\left|c_{0}\right|=\left|c_{n}\right| \neq 0$.

ДоказАтельство. Можно считать, что $M(P)=1$ и $P(0) \neq 0$. Обозначим через $\zeta=\Phi(w)$ функцию, конформно и однолистно отображающую внешность отрезка $\gamma:=[0,1]$ на круг $|\zeta|<1$ так, что $\Phi(\infty)=0$ и $\Phi(0)=-1$. Тогда функция

$$
f(z)=\Phi(\overline{P(\bar{z})}) P\left(\frac{1}{z}\right)
$$

голоморфна на множестве

$$
G=\left\{z:|z|<1, \overline{P(\bar{z})} P\left(\frac{1}{z}\right) \notin \gamma\right\}
$$

и аналитически продолжима на множество

$$
E=\{z:|z|=1,|P(\bar{z})| \neq 0,|P(\bar{z})| \neq 1\} .
$$

Кроме того, $f(z) \neq 0$ в $G \backslash\{0\}$ и в некоторой окрестности начала координат имеет место разложение

$$
f(z)=\frac{1}{4 \bar{c}_{0} c_{n}} z^{n}+\cdots,
$$

где $c_{0}$ - свободный, а $c_{n}$ - старший коэффициент полинома $P$. Поэтому функция

$$
\frac{z^{n}}{f(z)}
$$

голоморфна на открытом множестве $G$. В точках границы этого множества модуль указанной функции не превосходит единицы. По принципу максимума модуля заключаем отсюда, что неравенство

$$
|f(z)| \geqslant\left|z^{n}\right|
$$

выполняется всюду на множестве $G$. Пусть теперь $z$ - произвольная фиксированная точка множества $E$. Учитывая неравенство (1.2), имеем

$$
\left|f^{\prime}(z)\right|=\frac{\partial|f(z)|}{\partial|z|}=\lim _{r \rightarrow 1} \frac{|f(z)|-|f(r z)|}{1-r} \leqslant \lim _{r \rightarrow 1} \frac{1-r^{n}}{1-r}=n .
$$


Элементарные вычисления показывают, что

$$
\left|f^{\prime}(z)\right|=2|P(\bar{z})|\left|\operatorname{Im} \frac{\bar{z} P^{\prime}(\bar{z})}{P(\bar{z})}\right|\left(1-|P(\bar{z})|^{2}\right)^{-1 / 2} .
$$

Таким образом, из (1.3) вытекает неравенство (1.1) для любых точек $z$ на окружности $|z|=1$. Случай равенства проверяется непосредственно. Теорема доказана.

Перепишем неравенство (1.1) в виде

$$
\left|\left(|P(z)|^{2}\right)_{\varphi}^{\prime}\right| \leqslant n \sqrt{|P(z)|^{2}\left(M^{2}(P)-|P(z)|^{2}\right)}, \quad z=e^{i \varphi} .
$$

Интегрируя последнее неравенство по дуге окружности $|z|=1$ длины не большей $\pi / N$, приходим к следующему соотношению между дискретной и равномерной нормой полинома на окружности:

$$
\max _{\omega^{N}=1}|P(\omega)| \geqslant \cos \frac{\pi n}{2 N} \max _{|z|=1}|P(z)|,
$$

где $N$ - натуральное число, большее степени $n$. В случае $N=2 n$ неравенство (1.4) совпадает с оценкой Шейл-Смолла [42]:

$$
\max _{\omega^{N}=1}|P(\omega)| \geqslant \sqrt{\frac{N-n}{N}} \max _{|z|=1}|P(z)| .
$$

При $N>2 n$ неравенство (1.4) усиливает оценку (1.5), а также оценку Е. Рахманова и Б. Шехтмана, полученную ранее в работе [43]. Более того, для любого $N$, кратного $n$, неравенство (1.4) точное. Знак равенства достигается в случае $P(z)=(z \exp (i \pi / N))^{n}+1$ (см. [20]). Неравенство (1.4) можно усилить с учетом старшего и свободного коэффициентов полинома $P$ (см. следствие 1.6 настоящей статьи).

В заключение рассмотрим применение принципа максимума модуля к неравенствам для рациональных функций с предписанными полюсами [9]. Следуя работам [2], [44]-[50], введем класс $\mathcal{R}_{m}\left(a_{1}, \ldots, a_{n}\right)$, состоящий из рациональных функций вида

$$
R(z)=\frac{P(z)}{\prod_{k=1}^{n}\left(z-a_{k}\right)},
$$

где $P(z)$ - алгебраический полином степени $m$ и $\left|a_{k}\right|>1, k=1, \ldots, n$. Экстремальная функция связана с произведением Бляшке

$$
B(z)=\prod_{k=1}^{n} \frac{1-\bar{a}_{k} z}{z-a_{k}},
$$

определенным в общем случае для любой совокупности полюсов $\left(a_{1}, \ldots, a_{n}\right)$, $\left|a_{k}\right| \neq 1, k=1, \ldots, n$. Наконец, обозначения $L(R), H(R)$ и $M(R)$ возьмем из введения данной статьи, где вместо полинома $P$ рассматривается рациональная функция $R$.

Tеорема 1.3. Для любой функиии $R$ класса $\mathcal{R}_{m}\left(a_{1}, \ldots, a_{n}\right), m \geqslant n$, и любой точки z на окружности $|z|=1$ справедливо неравенство

$$
\left|\operatorname{Im}\left(z R^{\prime}(z)\right)\right| \leqslant\left|\left(z^{m-n} B(z)\right)^{\prime}\right| \sqrt{(\operatorname{Re} R(z)-L(R))(H(R)-\operatorname{Re} R(z))} .
$$

Равенство в (1.6) достигается для функиий вида $R(z)=\alpha+\beta z^{m-n} B(z)$, где $\alpha$ и $\beta$ - произвольные комплексные числа, $\beta \neq 0$. 
ДоказАтельство. Обозначим через $\omega=\Phi(w)$ функцию, конформно и однолистно отображающую внешность отрезка $\gamma:=[2 L(R), 2 H(R)]$ на внешность единичного круга $|\omega|>1$ так, что $\Phi(\infty)=\infty$ и $\Phi(2 L(R))=-1$. Пусть $G-$ открытое множество $\{\zeta:|\zeta|<1, R(1 / \zeta)+\overline{R(\bar{\zeta})} \notin \gamma\}$, и пусть

$$
f(\zeta)=\Phi\left(R\left(\frac{1}{\zeta}\right)+\overline{R(\bar{\zeta})}\right), \quad \zeta \in G
$$

Тогда функция

$$
F(\zeta)=f(\zeta) \zeta^{m-n} \prod_{k=1}^{n} \frac{1-a_{k} \zeta}{\zeta-\bar{a}_{k}}
$$

доопределенная по непрерывности в точках $\zeta=0,1 / a_{k}, k=1, \ldots, n$, является голоморфной на множестве $G$. При приближении $\zeta$ к границе этого множества функция $F(\zeta)$ имеет предельные значения, не превосходящие по модулю единицы. Следовательно, по принципу максимума модуля, примененному в каждой подобласти множества $G$, всюду в $G$ имеем оценку $|F(\zeta)| \leqslant 1$, т. е.

$$
|f(\zeta)| \leqslant\left|\zeta^{n-m} \prod_{k=1}^{n} \frac{\zeta-\bar{a}_{k}}{1-a_{k} \zeta}\right|
$$

Поскольку функция $f$ переводит единичную окружность $|\zeta|=1$ в окружность $|\omega|=1$, то в тех точках окружности $|\zeta|=1$, где существует производная, из неравенства (1.7) следует, что

$$
\frac{\partial|f|}{\partial|\zeta|} \geqslant \frac{\partial}{\partial|\zeta|}\left|\zeta^{n-m} \prod_{k=1}^{n} \frac{\zeta-\bar{a}_{k}}{1-a_{k} \zeta}\right|=-\frac{\partial}{\partial|z|}\left|z^{m-n} \prod_{k=1}^{n} \frac{1-\bar{a}_{k} z}{z-a_{k}}\right|=-\left|\left(z^{m-n} B(z)\right)^{\prime}\right|,
$$

где $z=1 / \zeta,|z|=1$. Нетрудно вычислить также производную в левой части этого неравенства:

$$
\begin{aligned}
\frac{\partial|f|}{\partial|\zeta|} & =-\frac{\partial|f|}{\partial|z|}=-\left|\frac{d \Phi(R(z)+\overline{R(1 / \bar{z})})}{d z}\right| \\
& =-\frac{\left|\operatorname{Im}\left(z R^{\prime}(z)\right)\right|}{\sqrt{(\operatorname{Re} R(z)-L(R))(H(R)-\operatorname{Re} R(z))}} .
\end{aligned}
$$

Теорема доказана.

Заменяя в неравенстве (1.6) среднее геометрическое на среднее арифметическое, получаем

$$
\left|\operatorname{Im}\left(z R^{\prime}(z)\right)\right| \leqslant\left|\left(z^{m-n} B(z)\right)^{\prime}\right| \frac{H(R)-L(R)}{2} \leqslant\left|\left(z^{m-n} B(z)\right)^{\prime}\right| M(R)
$$

для любых функций $R \in \mathcal{R}_{m}\left(a_{1}, \ldots, a_{n}\right)$. Отсюда при $m=n$ легко вытекает следующее утверждение.

СлЕДСтвиЕ 1.1. Если функиия $R$ принадлежит классу $\mathcal{R}_{n}\left(a_{1}, \ldots, a_{n}\right)$, то

$$
\left|R^{\prime}(z)\right| \leqslant\left|B^{\prime}(z)\right| M(R)
$$

для любых точек z на окружности $|z|=1$. 
По-видимому, впервые неравенство (1.8) встречается в работах В. Н. Русака (см. [51]). Позднее оно с некоторыми уточнениями было доказано П. Борвейном, Т. Эрдели [45] и, независимо, С. Ли, Р. Мохапатрой, Р. Родригесом [46] с помощью специальных интерполяционных формул (см. также [2], [48], [52]).

ЗАмечАниЕ 1.1. Приведенное выше доказательство неравенства (1.6) справедливо также для рациональных функций $R(z)$ вида

$$
P(z) /\left[z^{s} \prod_{k=1}^{n+l}\left(z-a_{k}\right)\right]
$$

где $P$ - полином степени $m \geqslant n+l+2 s, l \leqslant n,\left|a_{k}\right|>1, k=1, \ldots, n$, а каждая из точек $a_{j}, j=n+1, \ldots, n+l$, симметрична относительно окружности $|z|=1$ некоторой точке $a_{k}, 1 \leqslant k \leqslant n$. Поэтому для этих функций также имеет место неравенство (1.6), где $B(z)$ остается прежним, а $z^{m-n}$ необходимо заменить на $z^{m-n-l-s}$. В частности, если вновь $P$ - полином степени $n$, а точки $a_{k}$, $\left|a_{k}\right| \neq 1, k=1, \ldots, n$, расположены произвольным образом (не обязательно по одну сторону от окружности), то, применяя сказанное к рациональной функции $R(z)+\overline{R(1 / \bar{z})}$, где $R(z)=P(z) / \prod_{k=1}^{n}\left(z-a_{k}\right)$, и переходя от вещественной части к модулю, получим ослабленный вариант неравенства П. Борвейна и Т. Эрдели (см. [2; с. 324], [45]), где вместо максимума берется сумма соответствующих чисел.

Неравенства бернштейновского типа на окружности и на отрезке тесно связаны между собой [2]. Отметим известные результаты, вытекающие из неравенства (1.6). Пусть точки $a_{k} \in \mathbb{C} \backslash[-1,1], k=1, \ldots, n$, и невещественные числа $a_{k}$ этой совокупности образуют сопряженные пары. Следуя [2], [50], определим числа $c_{k}, k=1, \ldots, n$, соотношениями

$$
a_{k}:=\frac{c_{k}+c_{k}^{-1}}{2}, \quad\left|c_{k}\right|<1, \quad k=1, \ldots, n,
$$

так что

$$
c_{k}=a_{k}-\sqrt{a_{k}^{2}-1}, \quad k=1, \ldots, n,
$$

где под корнем понимается подходящая ветвь. В этом случае положительная величина

$$
B_{n}(x):=\operatorname{Re} \sum_{k=1}^{n} \frac{\sqrt{a_{k}^{2}-1}}{a_{k}-x}=\sum_{k=1}^{n} \frac{1-\left|c_{k}\right|^{2}}{\left|\zeta-c_{k}\right|^{2}}, \quad x \in[-1,1]
$$

$(x=(\zeta+1 / \zeta) / 2)$, называется бернштейновским фактором и играет важную роль в указанных работах.

Пусть

$$
R(z)=P(z) / \prod_{k=1}^{n}\left(z-a_{k}\right)
$$

где $P(z)$ - полином с вещественными коэффициентами степени $m \geqslant n$. Тогда функция $Q(\zeta):=R((\zeta+1 / \zeta) / 2)$ имеет вид

$$
\widetilde{P}(\zeta) /\left[\zeta^{m-n} \prod_{k=1}^{n}\left(\zeta-c_{k}\right)\left(\zeta-\frac{1}{\bar{c}_{k}}\right)\right],
$$


где $\widetilde{P}(\zeta)$ - полином степени $2 m$. С учетом замечания 1.1 из неравенства (1.6) получим

$$
\left|\operatorname{Im}\left(\zeta Q^{\prime}(\zeta)\right)\right| \leqslant\left[m-n+\left|B^{\prime}(\zeta)\right|\right] \sqrt{(Q(\zeta)-L(Q))(H(Q)-Q(\zeta))},
$$

где $B(\zeta)=\prod_{k=1}^{n}\left[\left(1-\zeta / c_{k}\right) /\left(\zeta-1 / \bar{c}_{k}\right)\right],|\zeta|=1$. После несложных вычислений приходим к неравенству

$$
\left|R^{\prime}(x)\right| \sqrt{1-x^{2}} \leqslant\left[m-n+B_{n}(x)\right] \sqrt{\left(R(x)-\min _{[-1,1]} R(x)\right)\left(\max _{[-1,1]} R(x)-R(x)\right)},
$$

где $x \in(-1,1)$.

При $n=1, a_{1}=a>1$ и $|R(x)| \leqslant 1,-1 \leqslant x \leqslant 1$, имеем неравенство В. С. Виденского [52]

$$
\left|R^{\prime}(x)\right| \sqrt{1-x^{2}} \leqslant m-1+\frac{\sqrt{a^{2}-1}}{a-x}, \quad-1<x<1 .
$$

Легко видеть также, что при $n=m$ полученное неравенство эквивалентно одному из результатов, установленных в работе [44], обобщающему на случай рациональных функций известное неравенство Бернштейна-Сегё для тригонометрических полиномов (о других многочисленных обобщениях см. [2; ч. 7]).

1.2. Обобщения и дополнения леммы Шварца. Пусть функция $f$ голоморфна в круге $U:=\{z:|z|<1\}, f(0)=0$ и $|f|<1$ в $U$. Согласно классической лемме Шварца, для любой точки $z$ из круга $U$ справедливо неравенство

$$
|f(z)| \leqslant|z| \text {. }
$$

Следовательно, имеем неравенство

$$
\left|f^{\prime}(0)\right| \leqslant 1 \text {. }
$$

Знаки равенства (в первом неравенстве при $z \neq 0$ ) будут здесь иметь место только в случае, когда $f(z) \equiv c z,|c|=1$ [39]. Нам понадобятся следующие обобщения и дополнения выписанных неравенств. Если функция $f$ голоморфна в $U$ и $|f|<1$ в $U$, то в круге $U$ имеем точную оценку

$$
\left|f^{\prime}(z)\right|\left(1-|z|^{2}\right) \leqslant 1-|f(z)|^{2}
$$

причем знак равенства будет только в случае функции $f(z)=c(z+a) /(1+\bar{a} z)$, $|c|=1,|a|<1$ (см. [39; гл. 8, § 1]). Выписывая неравенство (5) из книги [39; гл. $8, \S 1]$ для функции $f(z) / z$, получаем обобщение леммы Шварца:

$$
|f(z)| \leqslant|z| \frac{|z|+\left|f^{\prime}(0)\right|}{1+\left|f^{\prime}(0)\right||z|}, \quad z \in U
$$

справедливое для всех голоморфных в круге $U$ функций $f, f(0)=0,|f|<1$ в $U$ [53]. Если, дополнительно, функция $f$ продолжается по непрерывности в точку $b,|b|=1$, так, что $|f(b)|=1$ и существует производная $f^{\prime}(b)$, то предельным переходом из (1.10) приходим к граничной лемме Шварца:

$$
\left|f^{\prime}(b)\right| \geqslant \frac{2}{1+\left|f^{\prime}(0)\right|} \geqslant 1
$$


[53; с. 3514-3515]. Равенство в (1.10) для вещественных положительных $z$ и в левой части неравенства (1.11) для $b=1$ достигается при $f(z)=z(z+a) /(1+$ $a z), 0 \leqslant a \leqslant 1$.

В случае дополнительного условия однолистности функции $f$ справедлива более сильная, чем (1.11), оценка

$$
\left|f^{\prime}(b)\right| \geqslant \sqrt{\frac{1}{\left|f^{\prime}(0)\right|}} \geqslant 1,
$$

вытекающая из различных результатов теории функций (см. об этом [54; комментарий после теоремы 2], а также статью А.Ю. Солынина [55; неравенство (2.15)]). В приложениях конформных отображений к неравенствам для полиномов и рациональных функций, как правило, фигурирует обратная к $f$ функция $z=F(w)$, для которой неравенство (1.12) дает

$$
\left|F^{\prime}(c)\right| \leqslant \sqrt{\frac{1}{\left|F^{\prime}(0)\right|}}, \quad c=f(b) .
$$

Можно предположить, что искусственное построение однолистной функции $F$ (по заданному "неоднолистному" полиному) ведет к более слабым неравенствам, чем те, которые могут быть получены с учетом неоднолистности. В общем случае функция $F$ не имеет однозначной обратной функции $f$ и, следовательно, мы не можем воспользоваться неравенствами (1.11), (1.12). Сказанное выше приводит к необходимости получения теорем искажения для произвольных голоморфных функций $F$, заданных на некотором подмножестве круга $|w|<1$ и отображающих его на круг $|z|<1$. В этой связи представляет интерес следующий простой результат [10]. Пусть функция $F$ голоморфна на открытом множестве $G, 0 \in G \subset\{w:|w|<1\}, F(w) \neq 0$, когда $w \in G \backslash\{0\}$; $|F(w)|<1$ при $w \in G$, и пусть в окрестности начала координат справедливо разложение

$$
F(w)=d_{n} w^{n}+\cdots, \quad d_{n} \neq 0 .
$$

Предположим, что все предельные граничные значения $|F(w)|$ в $G$ равны единице. Тогда

$$
|F(w)| \geqslant|w|^{n}, \quad w \in G .
$$

Если к тому же область $G$ имеет на своей границе некоторую дугу $\gamma$ окружности $|w|=1$, а функция $F$ отображает эту дугу на дугу окружности $|w|=1$, то для любой внутренней точки $c$ дуги $\gamma$ справедливо неравенство

$$
\left|F^{\prime}(c)\right| \leqslant \frac{n^{2}\left(\left|d_{n}\right|+1\right)}{(n+1)\left|d_{n}\right|+n-1} \leqslant n .
$$

Равенство в (1.14) достигается для функции $F(w)=d_{n} w^{n},\left|d_{n}\right|=1$, и области $G=\{w:|w|<1\}$.

В условиях неравенства (1.14) рассмотрим множество $\widetilde{G}:=\{w: w \in G$, $|\widetilde{F}(w)| \neq 1\}$, где $\widetilde{F}(w)=F(w) / w^{n-1}$. Открытое множество $\widetilde{G}$ является объединением непересекающихся областей, совокупность которых обозначим через $\{D\}$. Функция $z=\widetilde{F}(w)$ голоморфна в каждой области $D$ из $\{D\}$. Предельные граничные значения модуля этой функции в $D$ не меньше единицы. 
Кроме того, $\widetilde{F}(0)=0, \widetilde{F}^{\prime}(0)=d_{n} \neq 0$ и $\widetilde{F}(w) \neq 0$ при $w \in \widetilde{G} \backslash\{0\}$. Используя принцип аргумента, легко заключить, что каждая область $D$ совокупности $\{D\}$ либо отображается функцией $z=\widetilde{F}(w)$ в $|z|>1$, либо конформно и однолистно отображается этой функцией на круг $|z|<1$ (ср. [8; лемма 2.1]). Пусть точка $c \in \gamma$ определена выше. Если $c \in \partial D$ и $\widetilde{F}(D) \subset\{z:|z|>1\}$, то в этой точке

$$
0 \geqslant \frac{\partial|\widetilde{F}|}{\partial|w|}=\frac{\partial|F|}{\partial|w|}-(n-1)=\left|F^{\prime}(c)\right|-(n-1) .
$$

В случае $c \in \partial D$ и $\widetilde{F}(D)=\{z:|z|<1\}$ из неравенства (1.13), примененного к функции $\widetilde{F}$, следует, что

$$
\left|F^{\prime}(c)\right| \leqslant n-1+\sqrt{\frac{1}{\left|d_{n}\right|}} .
$$

Таким образом, в любом случае выполняется неравенство (1.15). Сравнивая это неравенство с неравенством (1.14), элементарно заключаем, что при $\left|d_{n}\right|<$ $(n-1)^{2}$ оно слабее $(1.14)$, а при $\left|d_{n}\right|>(n-1)^{2}$ оно сильнее этого неравенства. Приложения неравенств (1.12) и (1.15) будут даны во второй главе данного обзора.

В качестве дополнения к граничной лемме Шварца (1.11) приведем следующий результат [54; теорема 2]. Пусть функция $f$ голоморфна в $U,|f|<1$ в $U$, и пусть эта функция и ее производная определены также в граничных точках $z_{k}$ таких, что точки $f\left(z_{k}\right)$ расположены на единичной окружности под равными углами, $k=1, \ldots, m, m \geqslant 2$. Тогда справедливо неравенство

$$
\left|\prod_{k=1}^{m} f^{\prime}\left(z_{k}\right)\right| \geqslant 1
$$

с равенством для функции $f(z) \equiv c z,|c|=1$.

Другие обобщения и дополнения леммы Шварца представлены в недавних обзорах [56], [57], а также в статьях [11], [54].

\section{3. Применение леммы Шварца к неравенствам для полиномов.} Прямое применение неравенств (1.9)-(1.11), (1.16) к специально построенным произведениям Бляшке приводит к новым результатам для некоторых классов целых функций. Рассмотрим этот подход в случае полиномов с нулями в единичном круге [15]. Обозначим через $\mathcal{P}_{n}$ класс всех полиномов вида

$$
P(z)=c_{n} \prod_{k=1}^{n}\left(z-\alpha_{k}\right)
$$

для которых $c_{n} \neq 0$ и $\left|\alpha_{k}\right| \leqslant 1, k=1, \ldots, n, n \geqslant 2$. Всюду ниже в этом разделе

$$
B(z)=\frac{P(z)}{z^{n-1} \overline{P(1 / \bar{z})}}=\frac{c_{n}}{\bar{c}_{n}} z \prod_{k=1}^{n} \frac{z-\alpha_{k}}{1-\bar{\alpha}_{k} z} .
$$

Непосредственно из неравенства (1.10), примененного к функции $B(z)$, вытекает следующая теорема. 
ТеОрема 1.4. Если полином $P(z)=c_{0}+\cdots+c_{n} z^{n}$ принадлежит классу $\mathcal{P}_{n}$, то для любой точки z из круга $U$ справедливо неравенство

$$
\left|\frac{P(z)}{P(1 / \bar{z})}\right| \leqslant|z|^{n} \frac{|z|\left|c_{n}\right|+\left|c_{0}\right|}{\left|c_{n}\right|+|z|\left|c_{0}\right|} .
$$

Равенство достигается, например, для полиномов $P$ с нулям на единичной окружности $|z|=1$.

Пусть теперь полином $P(z)=c_{0}+\cdots+c_{n} z^{n}$ не имеет нулей в круге $U$. Тогда полином $Q(z):=z^{n} P(1 / z)=c_{n}+\cdots+c_{0} z^{n}$ принадлежит классу $\mathcal{P}_{n}$. Из теоремы 1.4 следует, что для любой точки $z \mathrm{c}|z|>1$ выполняется неравенство

$$
\left|P\left(\frac{1}{\bar{z}}\right)\right| \geqslant|P(z)| \frac{1}{|z|^{n}} \frac{|z|\left|c_{0}\right|+\left|c_{n}\right|}{\left|c_{0}\right|+|z|\left|c_{n}\right|} .
$$

Предположим дополнительно, что $M(P)=1$. Тогда

$$
|P(z)|+|z|^{n}\left|P\left(\frac{1}{\bar{z}}\right)\right| \leqslant 1+|z|^{n}
$$

(см., например, [58; с. 550]). Вместе с предыдущим неравенством это дает следующий результат.

СлеДСТвиЕ 1.2. Если полином $P(z)=c_{0}+\cdots+c_{n} z^{n}, n \geqslant 2$, не имеет нулей в круге $U$ и $M(P)=1$, то для любого $r>1$ справедливо неравенство

$$
\max \{|P(z)|:|z|=r\} \leqslant \frac{\left(1+r^{n}\right)\left(\left|c_{0}\right|+r\left|c_{n}\right|\right)}{(1+r)\left(\left|c_{0}\right|+\left|c_{n}\right|\right)}
$$

с равенством для полиномов $P(z)=\left(\mu+\nu z^{n}\right) / 2,|\mu|=|\nu|=1$.

Поскольку правая часть последнего неравенства не превосходит $\left(1+r^{n}\right) / 2$, то мы имеем уточнение классического неравенства Н. Энкени и Т. Ривлина [59].

Теорема 1.5. Для полиномов $P(z)=c_{0}+\cdots+c_{n} z^{n}$ класса $\mathcal{P}_{n}$ в каждой точке $z$ окружности $|z|=1$, где $P(z) \neq 0$, выполняется неравенство

$$
\operatorname{Re} \frac{z P^{\prime}(z)}{P(z)} \geqslant \frac{n-1}{2}+\frac{\left|c_{n}\right|}{\left|c_{0}\right|+\left|c_{n}\right|} .
$$

Равенство в (1.17) имеет место для полиномов $P$ с нулями на единичной окружности $|z|=1$ для любых $z,|z|=1, P(z) \neq 0$.

ДокАзАтЕльство. Применение неравенства (1.11) к функции $B(z)$ дает

$$
\left|B^{\prime}(z)\right| \geqslant \frac{2}{1+\left|c_{0} / c_{n}\right|}
$$

для любых точек $z$ на окружности $|z|=1$. С другой стороны, для таких точек $z$ при $P(z) \neq 0$ имеем

$$
\left|B^{\prime}(z)\right|=\frac{z B^{\prime}(z)}{B(z)}=2 \operatorname{Re} \frac{z P^{\prime}(z)}{P(z)}-n+1,
$$

что приводит к неравенству (1.17). Если теперь все нули полинома $P$ расположены на единичной окружности, то $\left|c_{0}\right|=\left|c_{n}\right|$ и $B(z) \equiv c z,|c|=1$. Поэтому в последнем неравенстве и, следовательно, в неравенстве (1.17) имеет место знак равенства. Теорема доказана. 
Полученное неравенство несколько усиливает неравенство (14) работы [7]. Из неравенства (1.17) вытекает также следующее усиление классического неравенства П. Турана [60]:

$$
M\left(P^{\prime}\right) \geqslant M(P)\left[\frac{n}{2}+\frac{1}{2} \frac{\left|c_{n}\right|-\left|c_{0}\right|}{\left|c_{n}\right|+\left|c_{0}\right|}\right] .
$$

ТеОрема 1.6. Пусть $P$ - полином класса $\mathcal{P}_{n}$. Тогда для любых точек $z_{k}$, $k=1,2,3$, единичной окружности $|z|=1$, отличных от нулей полинома $P$, справедливо неравенство

$$
\prod_{k=1}^{3}\left(\operatorname{Re} \frac{z_{k} P^{\prime}\left(z_{k}\right)}{P\left(z_{k}\right)}-\frac{n}{2}\right) \geqslant \frac{1}{6 \sqrt{3}} \prod_{k=1}^{3}\left|\frac{z_{k}^{n} \overline{P\left(z_{k}\right)}}{P\left(z_{k}\right)}-\frac{z_{k+1}^{n} \overline{P\left(z_{k+1}\right)}}{P\left(z_{k+1}\right)}\right|,
$$

где полагаем $z_{4}=z_{1}$. Равенство достигается для полиномов $P(z)=c_{1} z+$ $c_{2} z^{2}+\cdots+c_{n} z^{n}$, все ненулевые корни которых расположены на единичной окружности.

ДокАзАТЕЛЬство вытекает из неравенства (1.16), примененного к суперпозиции функции $B(z) / z$ и подходящего дробно-линейного автоморфизма единичного круга (см. [27; теорема 12]).

Tеорема 1.7. Пусть $P(z)=\prod_{k=1}^{n}\left(z-\alpha_{k}\right),\left|\alpha_{k}\right| \leqslant 1, k=1, \ldots, n, n \geqslant 2$, и пусть $\beta_{k}, k=1, \ldots, n-1,-$ критические точки полинома $P$. Тогда справедливо неравенство

$$
\left|\sum_{k=1}^{n} \bar{\alpha}_{k} \prod_{k=1}^{n} \alpha_{k}-n \prod_{k=1}^{n-1} \beta_{k}\right| \leqslant 1-\left|\prod_{k=1}^{n} \alpha_{k}^{2}\right| \leqslant 1 .
$$

Равенство достигается в случае $P(z)=c_{1} z+z^{n},\left|c_{1}\right|=1$.

ДокАЗАТЕЛЬСтво. Положим $P(z)=c_{0}+c_{1} z+\cdots+c_{n-1} z^{n-1}+z^{n}$. Непосредственными вычислениями получаем

$$
f(z):=\frac{B(z)}{z}=\frac{P(z)}{z^{n} \overline{P(1 / \bar{z})}}=c_{0}+\left(c_{1}-c_{0} \bar{c}_{n-1}\right) z+\cdots .
$$

Неравенство (1.9) при $z=0$ дает

$$
\left|c_{1}-c_{0} \bar{c}_{n-1}\right| \leqslant 1-\left|c_{0}\right|^{2} .
$$

Осталось воспользоваться формулами Виета:

$$
c_{0}=(-1)^{n} \prod_{k=1}^{n} \alpha_{k}, \quad c_{1}=(-1)^{n-1} n \prod_{k=1}^{n-1} \beta_{k} \quad \text { и } \quad c_{n-1}=-\sum_{k=1}^{n} \alpha_{k} .
$$

Случай равенства виден из приведенного доказательства. Теорема доказана.

СледСтвие 1.3. Если полином $P(z)=\prod_{k=1}^{n}\left(z-\alpha_{k}\right)$ принадлежит классу $\mathcal{P}_{n}$ $u \sum_{k=1}^{n} \alpha_{k}=0, m o\left|P^{\prime}(0)\right| \leqslant 1-|P(0)|^{2} \leqslant 1$. 
Неравенство $\left|P^{\prime}(0)\right| \leqslant 1$ в условиях следствия 1.3 получено ранее П. Павловским другим путем (ср. [61; с. 4463-4464]).

СлЕДСТвиЕ 1.4. Если полином $P(z)=\prod_{k=1}^{n}\left(z-\alpha_{k}\right)$ принадлежит классу $\mathcal{P}_{n}$ и если $\sum_{k=1}^{n} \alpha_{k}=0$, либо $\prod_{k=1}^{n} \alpha_{k}=0$, то для критических точек $\beta_{k}, k=1, \ldots$, $n-1$, этого полинома, выполняется неравенство

$$
\min _{1 \leqslant k \leqslant n-1}\left|\beta_{k}\right| \leqslant\left(\frac{1}{n}\right)^{1 /(n-1)} .
$$

Равенство достигается в случае $P(z)=-z+z^{n}$.

В [13; теорема 3$]$ показано, что если $\beta_{k}, k=1, \ldots, n-1,-$ критические точки полинома $P(z)=c_{1} z+\cdots+z^{n}$ класса $\mathcal{P}_{n}$, то

$$
\sqrt[n-1]{\prod_{k=1}^{n-1}\left|P\left(\beta_{k}\right)\right|} \leqslant(n-1)\left|\frac{c_{1}^{2}}{n^{n}}\right|^{1 /(n-1)}
$$

с равенством в случае $P(z)=c_{1} z+z^{n}$, где $\left|c_{1}\right|=1$. С другой стороны, из теоремы 1.7 следует, что $\left|c_{1}\right| \leqslant 1$. Отсюда вытекает следующее утверждение.

СлеДСТВИЕ 1.5. Если $\beta_{k}, k=1, \ldots, n-1,-$ критические точки полинома $P(z)=c_{1} z+\cdots+z^{n}$ класса $\mathcal{P}_{n}$, то имеют место неравенства

$$
\prod_{k=1}^{n-1}\left|P\left(\beta_{k}\right)\right| \leqslant \frac{(n-1)^{n-1}}{n^{n}}, \quad \sqrt[n-1]{\prod_{k=1}^{n-1}\left|\frac{P\left(\beta_{k}\right)}{\beta_{k}}\right|} \leqslant \frac{n-1}{n} .
$$

Равенство в обоих случаях достигается для полинома $P(z)=-z+z^{n}$.

Следующее утверждение усиливает теорему 1.2 данного обзора.

ТЕОРема 1.8 [19]. Для любого полинома $P(z)=c_{0}+c_{1} z+\cdots+c_{n} z^{n}, n \geqslant 2$, и любой точки z на окружности $|z|=1$, в которой $|P(z)|$ отличен от $m(P)$ и $M(P)$, справедливы неравенства

$$
\begin{aligned}
& \frac{\left|\left(|P(z)|^{2}\right)_{\varphi}^{\prime}\right|}{\sqrt{\left(|P(z)|^{2}-m^{2}(P)\right)\left(M^{2}(P)-|P(z)|^{2}\right)}} \leqslant \lambda_{n} \\
& \quad:=\frac{n^{2}\left(M^{2}(P)-m^{2}(P)+4\left|c_{0} c_{n}\right|\right)}{(n+1)\left(M^{2}(P)-m^{2}(P)\right)+4\left|c_{0} c_{n}\right|(n-1)} \leqslant n .
\end{aligned}
$$

Равенства в левой и правой части (1.18) достигаются в случае $P(z)=c_{0}+$ $c_{n} z^{n}$ для любих точек $z,|z|=1, z^{n} \neq \pm c_{0} / c_{n}$, где $c_{0}$ и $c_{n}$ - произвольные комплексные числа, удовлетворяющие условию $\left|c_{0}\right|=\left|c_{n}\right| \neq 0$.

ДокАЗАТЕЛЬСтво. Можно считать, что $c_{0} \neq 0$ и $c_{n} \neq 0$. В этих условиях $m(P)<M(P)$. Действительно, в противном случае полином $P$ отображает окружность $|z|=1$ в себя и, ввиду того, что $P(\infty)=\infty$, принцип симметрии дает равенство $P(0)=0$. Это противоречит допущению $P(0)=c_{0} \neq 0$. Обозначим через $\zeta=\Phi(w)$ функцию, которая конформно и однолистно отображает 
внешность отрезка $\gamma=\left[m^{2}(P), M^{2}(P)\right]$ на круг $|\zeta|<1$ так, что $\Phi(\infty)=0$ и $\Phi\left(m^{2}(P)\right)=-1$. Тогда функция

$$
f(z)=\Phi\left(\overline{P(\bar{z})} P\left(\frac{1}{z}\right)\right)=\frac{M^{2}(P)-m^{2}(P)}{4 \bar{c}_{0} c_{n}} z^{n}+\cdots
$$

голоморфна на множестве

$$
G=\left\{z:|z|<1, \overline{P(\bar{z})} P\left(\frac{1}{z}\right) \notin \gamma\right\}
$$

и аналитически продолжима на множество

$$
E=\{z:|z|=1,|P(\bar{z})| \neq m(P),|P(\bar{z})| \neq M(P)\} .
$$

Кроме того, $f(z) \neq 0$ при $z \in G \backslash\{0\}$ и все предельные граничные значения $|f(z)|$ в $G$ равны единице. Согласно неравенству (1.14), в точках $z$ множества $E$ имеем

$$
\left|f^{\prime}(z)\right| \leqslant \lambda_{n} \leqslant n
$$

что равносильно неравенствам (1.18). Случаи равенства проще всего проверить, учитывая равенство в (1.14) (см. [19; доказательство теоремы 1]). Теорема доказана.

Интегрируя левое неравенство в $(1.18)$ на интервале $\left(\varphi_{1}, \varphi_{2}\right)$, приходим к следующему результату.

Теорема 1.9. Для любого полинома $P$ степени $n$, удовлетворяющего условию $m(P) \neq M(P)$, и для любых вещественных чисел $\varphi_{1}, \varphi_{2}$ справедливо неравенство

$$
\left|\arcsin \sqrt{\frac{\left|P\left(e^{i \varphi}\right)\right|^{2}-m^{2}(P)}{M^{2}(P)-m^{2}(P)}}\right|_{\varphi_{1}}^{\varphi_{2}} \mid \leqslant \frac{\lambda_{n}\left|\varphi_{1}-\varphi_{2}\right|}{2},
$$

где величина $\lambda_{n}$ определена в формулировке теоремы 1.8.

СлеДСТвИе 1.6 (ср. (1.4)). Для любого полинома $P$ степени $n$ и любого натурального числа $N \geqslant n$ выполняется неравенство

$$
\max _{\omega^{N}=1}|P(\omega)| \geqslant \cos \frac{\pi \lambda_{n}}{2 N} \max _{|z|=1}|P(z)| .
$$

СлЕДСТвиЕ 1.7 (см. [62; с. 154]). Предположим, что тригонометрическая сумма

$$
S_{n}(\theta)=\frac{A_{0}}{2}+\sum_{k=1}^{n} A_{k} \cos k \theta+B_{k} \sin k \theta
$$

удовлетворяет условиям $\left|S_{n}(0)\right| \leqslant 1,\left|S_{n}(\alpha)\right| \leqslant 1$ при некотором $\alpha, 0<\alpha<$ $\pi / n$. Если абсолютный максимум $M$ выражения $\left|S_{n}(\theta)\right|$ достигается на интервале $(0, \alpha)$, то

$$
M \leqslant \frac{1}{\cos (n \alpha / 2)} .
$$

ДокАЗАТЕЛЬство вытекает из теоремы 1.9 , примененной к полиному $P$ степени $2 n$, связанному с тригонометрической суммой $S_{n}(\theta)$ равенством

$$
S_{n}(\theta)=z^{-n} P(z), \quad z=e^{i \theta} .
$$


Теорема 1.10. Для любого полинома $P(z)=c_{0}+c_{1} z+\cdots+c_{n} z^{n}, \operatorname{Re} c_{n} \neq 0$, и любой точки z на отрезке $[-1,1]$, в которой $\operatorname{Re} P(z)$ отлична от $\bar{L}(P)$ и $\bar{H}(P)$, справедливо неравенство

$$
\begin{aligned}
& \frac{\left|\operatorname{Re} P^{\prime}(z) \sqrt{1-z^{2}}\right|}{\sqrt{(\operatorname{Re} P(z)-\bar{L}(P))(\bar{H}(P)-\operatorname{Re} P(z))}} \\
& \quad \leqslant \frac{n^{2}\left(\bar{H}(P)-\bar{L}(P)+2^{2-n}\left|\operatorname{Re} c_{n}\right|\right)}{(n+1)(\bar{H}(P)-\bar{L}(P))+(n-1) \cdot 2^{2-n}\left|\operatorname{Re} c_{n}\right|} \leqslant n .
\end{aligned}
$$

Равенство достигается в случае $P(z)=a T_{n}(z)+b$ для полинома Чебъшёва $T_{n}(z)=2^{n-1} z^{n}+\ldots$ илюбых вещественных $a \neq 0$, комплексных $b$ и $z \in[-1,1]$.

ДоказАтельство. Можно считать, что $n \geqslant 2$ и $\bar{L}(P) \neq \bar{H}(P)$. Обозначим через $\zeta=\Phi(\omega)$ функцию, конформно и однолистно отображающую внешность отрезка $[2 \bar{L}(P), 2 \bar{H}(P)]$ на круг $|\zeta|<1$ так, что $\Phi(\infty)=0, \Phi(2 \bar{L}(P))=-1$, и применим к функции

$$
F(w):=\Phi\left(P\left(\frac{w+1 / w}{2}\right)+\overline{P\left(\frac{\bar{w}+1 / \bar{w}}{2}\right)}\right)
$$

неравенство (1.14) в некоторой точке $c,|c|=1$. Требуемое неравенство получается отсюда заменой $z=(c+1 / c) / 2$. Случай равенства проверяется непосредственно. Теорема доказана.

Можно показать, что неравенство теоремы 1.10 усиливает классические неравенства Маркова и Бернштейна (см. [8; комментарий после теоремы 4.4]).

1.4. О целых функциях экспоненциального типа. Указанные в заглавии этого раздела функции представляют значительный интерес. K настоящему времени для них получено немало интересных результатов, и исследование этих функций продолжается до сих пор (см., например, [63]-[66]). Целыми функциями экспоненциального типа называются целые функции, порядок которых меньше 1, либо равен 1, но тогда тип конечен. Как обычно, через

$$
h_{f}(\theta)=\varlimsup_{r \rightarrow \infty} \frac{\log \left|f\left(r e^{i \theta}\right)\right|}{r}
$$

будем обозначать индикатор роста функции $f$. Рассмотрим класс $\mathscr{E}$ целых функций экспоненциального типа, удовлетворяющих следующим условиям: $f(z) \neq 0$ в верхней полуплоскости $\operatorname{Im} z>0$ и $h_{f}(\pi / 2)=h_{f}(-\pi / 2)$. Заметим, что если $f$ - целая функция экспоненциального типа, $f(z) \neq 0$ при $\operatorname{Im} z>0$ и $h_{f}(\pi / 2)=\tau, h_{f}(-\pi / 2)=\sigma$ (т. е. $f \in \mathscr{A}_{\sigma}^{\tau}$; см. работы [67], [35]), то функция $g(z)=f(z) e^{i(\tau-\sigma) z / 2}$ принадлежит классу $\mathscr{E}$. Применяя к функции $g$ следующие ниже теоремы, получим соответствующие утверждения для функций класса $\mathscr{A}_{\sigma}^{\tau}$. Пусть теперь $f \in \mathscr{E}$. Тогда функция $\overline{f(\bar{z})}$ принадлежит известному классу $P[63 ;$ с. 217]. Из представления функций класса $P$ следует, что функция

$$
L(z):=\frac{f(z)}{\overline{f(\bar{z})}}=c \prod_{k} \frac{1-z / \alpha_{k}}{1-z / \bar{\alpha}_{k}},
$$


где $|c|=1, \operatorname{Im} \alpha_{k} \leqslant 0$ и $\sum_{k}\left|\operatorname{Im} \frac{1}{\alpha_{k}}\right|<\infty$ [63; с. 219]. Если функция $f$ имеет только вещественные нули, то $L(z) \equiv c,|c|=1$; в общем случае $|L(z)| \leqslant 1$ при $\operatorname{Im} z<0$.

Пусть $f \in \mathscr{E}$ и $z_{0}$ - фиксированная точка в нижней полуплоскости: $\operatorname{Im} z_{0}<0$. Ввиду (1.19) суперпозиция

$$
B(\zeta)=\zeta L(z(\zeta)), \quad \text { где } \quad z(\zeta)=\frac{z_{0}+\zeta \bar{z}_{0}}{1+\zeta},
$$

является произведением Бляшке, переводящим круг $|\zeta|<1$ в себя. Данная функция использовалась в работе [35] для построения конформного и однолистного отображения в соответствии с предложенным в [8] подходом к получению неравенств для многолистных функций. Однако в ряде частных случаев эффективнее непосредственное применение неравенств (1.9)-(1.11), например, к функциям $L(z(\zeta))$ и $B(\zeta)$. Таким образом, эти два подхода дополняют друг друга. Заметим также, что наряду с функцией $B(\zeta)$ можно рассматривать другое произведение Бляшке

$$
\frac{L(z(\zeta))-L\left(z_{0}\right)}{1-\overline{L\left(z_{0}\right)} L(z(\zeta))}
$$

к которому применены все следующие ниже выкладки для $B(\zeta)$.

Теорема 1.11. Если $f \in \mathscr{E}$, то для любой точки $z, \operatorname{Im} z<0, f(z) \neq 0$, справедливо неравенство

$$
2|\operatorname{Im} z| \frac{f^{\prime}(z)}{f(z)}-\frac{\overline{f^{\prime}(\bar{z})}}{\overline{f(\bar{z})}} \mid \leqslant \frac{\left|f^{2}(\bar{z})\right|-\left|f^{2}(z)\right|}{|f(z) f(\bar{z})|} .
$$

Равенство достигается в том и только том случае, когда все нули функици $f$, за исключением, может быть, одного, лежат на вещественной оси.

ДокАЗАТЕЛЬство. Если $L(z) \not \equiv$ const, то, применяя неравенство (1.9) к функции $L(z(\zeta))$ в точке $\zeta=0$, получаем

$$
\left|\frac{L^{\prime}\left(z_{0}\right)}{L\left(z_{0}\right)}\right|\left|2 \operatorname{Im} z_{0}\right| \leqslant \frac{1}{\left|L\left(z_{0}\right)\right|}-\left|L\left(z_{0}\right)\right|,
$$

что дает требуемое неравенство при $z_{0}=z$. Равенство имеет место в том и только том случае, когда

$$
L(z(\zeta))=c \frac{\zeta+a}{1+\bar{a} \zeta}
$$

при некоторых $c,|c|=1$, и $a,|a|<1$. Ввиду (1.19) это соответствует случаю, когда один нуль функции $f$ лежит в нижней полуплоскости, а все остальные нули (если таковые имеются) - на вещественной оси. Если теперь $L(z) \equiv$ const, то необходимо $|L(z)| \equiv 1$, и мы имеем случай равенства, в котором все нули функции $f$ расположены на вещественной оси. Теорема доказана.

Из неравенства (1.10), примененного к функции $B(\zeta)$, вытекает следующая теорема. 
Теорема 1.12. Если $f \in \mathscr{E}$, то для любых точек z и $\zeta$ в нижней полуплоскости выполняется неравенство

$$
|z-\bar{\zeta}|(|f(z) f(\bar{\zeta})|-|f(\bar{z}) f(\zeta)|) \leqslant|z-\zeta|(|f(\bar{z}) f(\bar{\zeta})|-|f(z) f(\zeta)|) .
$$

Равенство достигается, например, для функиий $f$, все нули которых расположены на вещественной оси.

Заметим, что ввиду (1.19) для любой функции $f$ класса $\mathscr{E}$ в точках $x$ на вещественной оси имеет место неравенство

$$
\operatorname{Im} \frac{f^{\prime}(x)}{f(x)}=\sum_{k} \frac{\operatorname{Im} \alpha_{k}}{\left|\alpha_{k}-x\right|^{2}} \leqslant 0 .
$$

Отсюда следует, что

$$
(\arg f(x))^{\prime}=\operatorname{Im} \frac{f^{\prime}(x)}{f(x)} \leqslant 0 .
$$

Здесь и ниже $(\arg f(x))^{\prime}$ означает частную производную $\partial \arg f / \partial x$, вычисленную в точке $x$ на вещественной оси. Если дополнительно известны значения функции $f$ в некоторых симметричных точках $\zeta$ и $\bar{\zeta}$, то неравенство $(1.20)$ допускает следующее усиление.

Теорема 1.13. Пусть $f \in \mathscr{E}$, и пусть $x$-вещественное, а $\zeta$-комплексное число в нижней полуплоскости. Тогда

$$
(\arg f(x))^{\prime} \leqslant \frac{|f(\bar{\zeta})|-|f(\zeta)|}{|f(\bar{\zeta})|+|f(\zeta)|} \frac{\operatorname{Im} \zeta}{|x-\zeta|^{2}} .
$$

Равенство в (1.21) имеет место для функиий $f$ класса Е с нулями на вещественной оси.

ДокАзАТЕЛьство. Применение неравенства (1.11) к функции

$$
B(\zeta)=\zeta L(z(\zeta))=L\left(z_{0}\right) \zeta+\cdots
$$

дает

$$
\left|B^{\prime}(\zeta)\right| \geqslant \frac{2}{1+\left|L\left(z_{0}\right)\right|}=1+\frac{1-\left|L\left(z_{0}\right)\right|}{1+\left|L\left(z_{0}\right)\right|}
$$

для любых $\zeta,|\zeta|=1, \zeta \neq-1$. С другой стороны, в точке $\zeta$, для которой $x=z(\zeta)$, имеем

$$
\left|B^{\prime}(\zeta)\right|=1+\frac{\zeta L^{\prime}(z(\zeta)) z^{\prime}(\zeta)}{L(z(\zeta))}=1+\frac{\zeta(z) L^{\prime}(z(\zeta))}{\zeta^{\prime}(z) L(z(\zeta))}=1+\frac{\left|x-z_{0}\right|^{2}}{\operatorname{Im} z_{0}} \operatorname{Im} \frac{f^{\prime}(x)}{f(x)},
$$

где $\zeta(z)=\left(z_{0}-z\right) /\left(z-\bar{z}_{0}\right)-$ функция, обратная к $z=z(\zeta)$. Неравенство (1.21) доказано (при $\left.\zeta=z_{0}\right)$. В случае, когда все нули функции $f$ расположены на вещественной оси, мы имеем $L(z) \equiv c,|c|=1$ (см. (1.19)). Поэтому $B(\zeta)=c \zeta$ и в неравенствах (1.11) и (1.21) имеет место знак равенства. Теорема доказана.

Теоремы 1.11 и 1.13 дают усиление соответственно теорем 5 и 3 работы [35], полученных другим путем. Сочетание неравенства (1.21) с некоторыми результатами Б. Я. Левина [63] и Т. Г. Генчева [67] для целых функций экспоненциального типа приводит к уточнению классических теорем в разных направлениях (см. применение теоремы 3 в [35; следствия 2-4]). 
Теорема 1.14. Если $f \in \mathscr{E}$, то для любъх различных точек $x_{1}$ и $x_{2}$ на вещественной оси справедливо неравенство

$$
\left(\arg f\left(x_{1}\right)\right)^{\prime}\left(\arg f\left(x_{2}\right)\right)^{\prime}\left|x_{1}-x_{2}\right|^{2} \geqslant \sin ^{2} \arg \frac{f\left(x_{1}\right)}{f\left(x_{2}\right)} .
$$

Равенство в (1.22) достигается для функиий $f$ класса $\mathscr{E}$, у которых в нижней полуплоскости имеется только один нуль $z_{0}=\left(x_{1}+x_{2}\right) / 2-i\left|x_{1}-x_{2}\right| / 2$.

ДокАЗАТЕЛЬство. Пусть $L\left(x_{1}\right) \neq L\left(x_{2}\right)$, и пусть точки $\zeta_{k}$ таковы, что $x_{k}=$ $z\left(\zeta_{k}\right), k=1,2$. Обозначим через $\Psi(w)$ один из дробно-линейных автоморфизмов круга $|w|<1$ на себя, переводящих точки $L\left(x_{k}\right), k=1,2$, в точки \pm 1 . Из неравенства (1.16), примененного к суперпозиции $\Psi(L(z(\zeta)))$, следует, что

$$
\left|\Psi^{\prime}\left(L\left(x_{1}\right)\right) \Psi^{\prime}\left(L\left(x_{2}\right)\right) L^{\prime}\left(x_{1}\right) L^{\prime}\left(x_{2}\right) z^{\prime}\left(\zeta_{1}\right) z^{\prime}\left(\zeta_{2}\right)\right| \geqslant 1 .
$$

Полагая $z_{0}$ в определении функции $z(\zeta)$ равной $\left(x_{1}+x_{2}\right) / 2-i\left|x_{1}-x_{2}\right| / 2$ и используя инвариантность известного функционала при дробно-линейных отображениях, заключаем из (1.23), что

$$
\left|L^{\prime}\left(x_{1}\right) L^{\prime}\left(x_{2}\right)\right|\left|x_{1}-x_{2}\right|^{2} \geqslant\left|L\left(x_{1}\right)-L\left(x_{2}\right)\right|^{2} .
$$

Это же неравенство верно при $L\left(x_{1}\right)=L\left(x_{2}\right)$. После несложных вычислений приходим к неравенству (1.22). Если теперь функция $f$ имеет в нижней полуплоскости только один нуль $z_{0}=\left(x_{1}+x_{2}\right) / 2-i\left|x_{1}-x_{2}\right| / 2$, то ввиду (1.19)

$$
L(z) \equiv c \frac{z-z_{0}}{z-\bar{z}_{0}}, \quad|c|=1 .
$$

Таким образом, $L(z(\zeta))$ есть поворот вокруг точки $\zeta=0$. Непосредственно убеждаемся, что $\zeta_{1}=-\zeta_{2}$ и, следовательно, $\Psi$ также является поворотом вокруг начала. Отсюда вытекает равенство в (1.23) и (1.22). Теорема доказана.

Аналогично доказывается “трехточечная” теорема искажения для функций класса $\mathscr{E}$ и для любых различных точек $x_{1}, x_{2}$ и $x_{3}$ на вещественной оси.

В работе [66] установлено замечательное неравенство

$$
|f(z)|>\mu \text { при } \operatorname{Im} z>0,
$$

справедливое для всех целых функций $f$ экспоненциального типа, отличных от постоянной, не имеющих нулей в верхней полуплоскости и удовлетворяющих условиям $h_{f}(\pi / 2)=0,|f(x)| \geqslant \mu>0$ на вещественной оси. В [66] даны также интересные приложения этого неравенства. Новые приложения можно получить, применяя неравенства (1.9), (1.10) к функции $w=\mu / f(z(\zeta)),|\zeta|<1$, где $z(\zeta)$ определена выше, но с $\operatorname{Im} z_{0}>0$. Например, из неравенства (1.9), аналогично доказательству теоремы 1.11, получаем следующий результат:

$$
\left|f^{\prime}(z)\right| \leqslant \frac{\left|f^{2}(z)\right|-\mu^{2}}{2 \mu \operatorname{Im} z}, \quad \operatorname{Im} z>0 .
$$

В заключение отметим, что если все нули полинома $P$ степени $n$ лежат в круге $|z| \leqslant 1$, то функция $f(z)=e^{i n z / 2} P\left(e^{-i z}\right)$ принадлежит классу $\mathscr{E}$. Применение к этой функции предыдущих теорем приводит к некоторым утверждениям для полинома $P$. Однако более сильные результаты получаются, если повторить предыдущие доказательства теорем, но уже применительно к произведению Бляшке, построенному по полиному $P$. 
1.5. Дополнение к принципу Линделёфа с приложениями к полиномам и рациональным функциям. Функцию Грина $g_{D}(z, \zeta)$ области $D$ называют классической, если она обращается в нуль на границе $D$. В этом случае доопределим функцию $g_{D}(z, \zeta)$ нулем в дополнении $\overline{\mathbb{C}}_{z} \backslash D$. Следующее утверждение принято называть приниипом Линделёба [68]. Пусть области $D$ и $G$ расширенной комплексной плоскости имеют классические функции Грина, и пусть функция: $f: D \rightarrow G$ отлична от постоянной и мероморфна в $D$. Тогда для любой фиксированной конечной точки $w_{0}$, принадлежащей $f(D)$, справедливо неравенство

$$
g_{G}\left(f(z), w_{0}\right) \geqslant \sum_{k \geqslant 0} g_{D}\left(z, z_{k}\right), \quad z \in D
$$

где $z_{0}, z_{1}, \ldots, z_{k}, \ldots$ - нули функции $f-w_{0}$, причем каждый из них учитывается столько раз, какова его кратность. Равенство в (1.24) в какой-либо точке $z \in D \backslash \bigcup_{k \geqslant 0}\left\{z_{k}\right\}$ влечет за собой равенство в (1.24) во всей области $D$. Даже ослабленный вариант этого принципа

$$
g_{G}\left(f(z), w_{0}\right) \geqslant g_{D}\left(z, z_{0}\right)
$$

является существенным обобщением леммы Шварца и имеет многочисленные приложения в геометрической теории функций (см., например, [41], [68]). Распространение принципа Линделёфа на случай функций Робена вместо функций Грина дано в недавней работе [69]. Обозначим через $r(D, \zeta)$ внутренний радиус области $D$ относительно точки $\zeta \in D$, определяемый соотношением

$$
g_{D}(z, \zeta)=-\log |z-\zeta|+\log r(D, \zeta)+o(1), \quad z \rightarrow \zeta .
$$

В работе [70] И. П. Митюк заметил, что из неравенства (1.24) вытекает неравенство

$$
r\left(f(D), w_{0}\right) \geqslant\left|a_{p}\right| r^{p}\left(D, z_{0}\right) \exp \left\{\sum_{k \geqslant 1} g_{D}\left(z_{0}, z_{k}\right)\right\},
$$

где $f(z)=w_{0}+a_{p}\left(z-z_{0}\right)^{p}+\cdots-$ голоморфная функция в области $D$. Более того, И. П. Митюк показал эффективность применения последнего неравенства в сочетании с методами симметризации [70]-[73]. Применение неравенства (1.25) к полиному $(f=P)$ дает следующий результат (см. [23; теорема 9]).

Теорема 1.15. Если $P$ - полином степени $n$, то для любой точки $z$, удовлетворяющей условию $\{\zeta: P(\zeta)=P(z)\} \subset U$, справедливо неравенство

$$
M^{2}(P)-|P(z)|^{2} \geqslant|z|^{n}\left|P\left(\frac{1}{\bar{z}}\right)-P(z)\right| M(P) .
$$

Равенство достигается в случае $P(z)=c z^{n}, c \neq 0$.

В пособии [72] впервые указано на необходимость изучения неравенств, обратных (1.24), (1.25). Именно, следуя [72], рассмотрим функцию $f(z)=w_{0}+$ $a_{p}\left(z-z_{0}\right)^{p}+\cdots$, голоморфную в области $D$, и пусть $A$ - множество предельных точек последовательностей $\left\{f\left(z_{\nu}^{\prime}\right)\right\}$, когда $z_{\nu}^{\prime} \in D, z_{\nu}^{\prime} \rightarrow \partial D, \nu \rightarrow \infty$. Фиксируем 
конечную точку $w_{0} \notin A$ и обозначим через $G$ связную компоненту дополнения множества $A$, содержащую точку $w_{0}$. Тогда

$$
r\left(G, w_{0}\right) \leqslant\left|a_{p}\right| r^{p}\left(D, z_{0}\right) \exp \left\{\sum_{k \geqslant 1} g_{D}\left(z_{0}, z_{k}\right)\right\}
$$

причем равенство в (1.26) имеет место в том и только том случае, когда $G=$ $f(D)$ и функция $f$ производит полное $n$-кратное накрытие области $G$ областью $D$ (здесь $n$ - суммарная кратность нулей функции $\left.f-w_{0}\right)$. Последнее означает, что при стремлении точки $z$ к границе области $D$ все предельные значения функции $f(z)$ принадлежат границе $G$ и каждой точке $w \in G$ соответствует ровно $n$ прообразов в области $D$ (с учетом кратности) [72; с. 22]. Сказанное выше можно перефразировать следующим образом: если $f(\partial D) \subset \overline{\mathbb{C}}_{w} \backslash G$, то справедливо неравенство (1.26). Заметим, что неравенство (1.25) выполняется при условии $f(D) \subset G$. Однако для неоднолистной функции $f$ противоположного включения $f(D) \supset G$ недостаточно для выполнения неравенства (1.26), как показывает пример функции $f(z)=z^{2}$ и областей $D=\mathbb{C}_{z} \backslash\{z=x+i y:-\infty \leqslant$ $x \leqslant-1, y=0\}$ и $G=\{w:|w|<R\}$ при любом $R>16\left(z_{0}=w_{0}=0\right)$. Следующее утверждение содержит неравенство (1.26) как следствие при $z \rightarrow z_{0}$ (см. [16; теорема 1]). Пусть области $D$ и $G$ имеют классические функции Грина, $D \subset \overline{\mathbb{C}}_{z}, G \subset \overline{\mathbb{C}}_{w}$, и пусть $w_{0}$ - фиксированная точка области $G$. Предположим, что функция $f$ является мероморфной в области $D$, принимает по меньшей мере один раз значение $w_{0}$ в $D$ и удовлетворяет условию $f(\partial D) \subset \overline{\mathbb{C}}_{w} \backslash G$ (т.е. при стремлении точки $z$ к границе области $D$ все предельные значения функции $f(z)$ лежат в дополнении области $G$ ). Тогда для любой точки $z \in D$ справедливо неравенство

$$
g_{G}\left(f(z), w_{0}\right) \leqslant \sum_{k=0}^{m} g_{D}\left(z, z_{k}\right)
$$

где $z_{0}, \ldots, z_{m}$ - все нули функции $f-w_{0}$ в случае $w_{0} \neq \infty$ и нули функции $1 / f$ при $w_{0}=\infty$, причем каждый нуль учитывается столько раз, какова его кратность. Равенство в (1.27) в какой-нибудь точке $z \in D \backslash \bigcup_{k=1}^{m}\left\{z_{k}\right\}$ влечет за собой равенство в (1.27) во всей области $D$ и выполняется тогда и только тогда, когда $G=f(D)$ и функция $f$ осуществляет полное $(m+1)$-кратное накрытие области $G$ областью $D$. Неравенство (1.27) впервые встречается в работе И. П. Митюка [74] при несколько иных ограничениях на функцию $f$. И. П. Митюк рассматривал (1.27) как вспомогательное неравенство для иного, нежели в [72], варианта доказательства оценки (1.26), дополняющей его ранний результат (1.25). Вместе с тем неравенство (1.27) является важным дополнением к принципу Линделёфа, и, как выяснилось много лет спустя, оно содержит, в частности, многие классические и современные неравенства для полиномов и рациональных функций [25]. Отметим, что из неравенства (1.27) вытекает следующее утверждение (ср. следствие 2 работы [25]). Пусть в предыдущих условиях область $D$ имеет на своей границе открытую аналитическую дугу Жордана $\gamma, \infty \notin \gamma$, ни одна точка которой не является предельной для граничных точек, не принадлежащих $\gamma$, а область $G$ имеет на своей границе такую же дугу Г. Предположим, что функция $f$ отображает дугу $\gamma$ на некоторую дугу 
$f(\gamma) \subset \Gamma$ так, что положительной ориентации $\gamma$ соответствует положительная ориентация на $\Gamma{ }^{1}$ Тогда для любой точки $z \in \gamma$ справедливо неравенство

$$
\left|f^{\prime}(z)\right| \frac{\partial g_{G}\left(f(z), w_{0}\right)}{\partial n} \leqslant \sum_{k=0}^{m} \frac{\partial g_{D}\left(z, z_{k}\right)}{\partial n},
$$

где $\partial / \partial n$ означает дифференцирование вдоль внутренней нормали к соответствующей граничной дуге. Равенство в какой-либо точке $z \in \gamma$ достигается лишь в случае, когда выполняется равенство в (1.27) (см. [16; следствие 1]).

Неравенство (1.27) можно трактовать как теорему покрытия [25], являющуюся далеко идущим обобщением теоремы 1.1. Неравенство (1.28) содержит многочисленные неравенства бернштейновского типа. Например, пусть $D=\overline{\mathbb{C}}_{z} \backslash E, G=\overline{\mathbb{C}}_{w} \backslash F$ и $w_{0}=\infty$, где $E$ и $F$ - некоторые компактные множества ненулевой емкости на вещественной оси. Предположим, что функция $f=R$ - рациональная функция степени $n$, являющаяся отношением двух вещественных полиномов, и пусть $R(E) \subset F$. Тогда неравенство (1.28) совпадает с неравенством А. Л. Лукашова (см. [75], [76]):

$$
\left|R^{\prime}(x)\right| \widetilde{\omega}_{F}(\infty, f(x)) \leqslant \sum_{k=1}^{n} \widetilde{\omega}_{E}\left(z_{k}, x\right),
$$

справедливого для любой внутренней точки $x$ компакта $E$. Здесь

$$
\widetilde{\omega}_{E}(z, x)=\frac{d}{d x}\left(\omega\left(z,[\inf E, x] \cap E, \overline{\mathbb{C}}_{z} \backslash E\right)\right),
$$

где $\omega(z, e, \Omega)$ - гармоническая мера множества $e \subset \partial \Omega$ в точке $z$ относительно области $\Omega(m=n)$. При доказательстве неравенства (1.29) А. Л. Лукашов существенно использует свой предыдущий результат из [75]. В работе [76] приводятся также утверждения, подтверждающие точность неравенства (1.29). Уточнения и дополнения этих результатов А. Л. Лукашова установлены С. И. Калмыковым в работе [32]. Выбирая конкретные компакты $E$ и $F$ в (1.29), получаем ряд известных неравенств С. Н. Бернштейна-Г. Сегё, Н. И. Ахиезера, В. С. Виденского, В.Н. Русака, М. Барана-В. Тотика и других математиков (см. [25], [76], а также замечания по истории вопроса в [75; §5]). В дополнение к этим неравенствам рассмотрим пример применения неравенства (1.28) в случае, когда $f=R$ - рациональная функция с ограничением на окружности. Предположим, что все полюсы $a_{k}, k=1, \ldots, n$, рациональной функции $R$ конечны и $M(R)=1$. Применяя неравенство (1.28) к функции $R(z)+\overline{R(1 / \bar{z})}$ в области $D=\{z:|z|>1\}$, приходим к неравенству

$$
\left|R^{\prime}(z)\right| \leqslant \sum_{k=1}^{n} \frac{\left.|1-| a_{k}\right|^{2} \mid}{\left|a_{k}-z\right|^{2}}
$$

справедливому для любых точек $z$ на окружности $|z|=1$ (см. [25; § 2]). В случае $\left|a_{k}\right|>1, k=1, \ldots, n$, неравенство (1.30) получено В. Н. Русаком (см. (1.8)).

\footnotetext{
13десь дуги $\gamma$ и Г являются граничными кривыми, при обходе которых соответствующая область остается слева, при этом граничные точки считаются различными, если они являются носителями различных достижимых граничных точек, а предельная точка понимается в соответствующей топологии.
} 
Отметим также, что применение неравенства (1.28) к специально подобранным мероморфным функциям ведет к неравенствам бернштейновского типа для полиномов с криволинейной мажорантой [32].

Рассмотрим приложения неравенств (1.26)-(1.28) к оценкам для полиномов с ограничениями на дугах окружности [28]. В последнее время таким полиномам уделяется значительное внимание [34], [77]-[80]. Ранее указанные полиномы изучались в работах [81], [82], причем в случае алгебраических полиномов речь шла об ограничениях на дугах окружности [82], а в эквивалентной задаче для тригонометрических полиномов ограничения приходились на отрезок, меньший чем период [81]. Всюду ниже в этом разделе Г - объединение конечного числа непересекающихся замкнутых невырожденных дуг единичной окружности $|z|=1, D=\overline{\mathbb{C}}_{z} \backslash \Gamma$,

$$
\begin{aligned}
& m=m(P, \Gamma)=\min \{|P(z)|: z \in \Gamma\}, \\
& M=M(P, \Gamma)=\max \{|P(z)|: z \in \Gamma\} .
\end{aligned}
$$

Tеорема 1.16. Пусть $P$ - полином степени $n$, и пусть $f(z)=P(z) \overline{P(1 / \bar{z})}$. Тогда для любой точки z выполняется неравенство

$$
\begin{aligned}
& \left|2 f(z)-M^{2}-m^{2}+2 \sqrt{\left(f(z)-M^{2}\right)\left(f(z)-m^{2}\right)}\right| \\
& \leqslant\left(M^{2}-m^{2}\right) \exp \left\{n\left(g_{D}(z, 0)+g_{D}\left(\frac{1}{\bar{z}}, 0\right)\right)\right\}
\end{aligned}
$$

(при любом выборе значения корня в левой части). Равенство в точке $z$, $z \neq 0, \infty u z \notin \Gamma$, при некотором значении корня достигается в том и только том случае, когда функиия $f$ осуществляет полное 2 -кратное накрытие области $\overline{\mathbb{C}}_{w} \backslash\left[\mathrm{m}^{2}, M^{2}\right]$ областью $D$.

ДокАЗАтельство. Области $D$ и $G=\overline{\mathbb{C}}_{w} \backslash\left[m^{2}, M^{2}\right]$ имеют классические функции Грина. Функция $f$ мероморфна в $D$ и имеет в области $D$ только два полюса: в точке $z=0$ и точке $z=\infty$, каждый порядка не более $n$. Кроме того, при стремлении точки $z$ к множеству $Г$ все предельные граничные значения функции $f$ лежат на отрезке $\left[m^{2}, M^{2}\right]$. Согласно неравенству $(1.27)$, в точках области $D$ выполняется

$$
g_{G}(f(z), \infty) \leqslant n\left(g_{D}(z, \infty)+g_{D}(z, 0)\right),
$$

причем равенство в точках $z \neq 0$ и $\infty$ имеет место тогда и только тогда, когда $G=f(D)$ и функция $f$ осуществляет полное $2 n$-кратное накрытие области $\overline{\mathbb{C}}_{w} \backslash\left[m^{2}, M^{2}\right]$ областью $D$. Из симметрии области $D$ относительно окружности $|z|=1$ получаем

$$
g_{D}(z, \infty)=g_{D}\left(\frac{1}{\bar{z}}, 0\right)
$$

Осталось заметить, что

$$
g_{G}(w, \infty)=\log \left|\frac{2 w-M^{2}-m^{2}}{M^{2}-m^{2}}+\sqrt{\left(\frac{2 w-M^{2}-m^{2}}{M^{2}-m^{2}}\right)^{2}-1}\right| .
$$

Теорема доказана. 
В случае, когда множество Г состоит из одной дуги

$$
\Gamma_{\alpha}:=\left\{z=e^{i \varphi}:-\alpha \leqslant \varphi \leqslant \alpha\right\}, \quad 0<\alpha \leqslant \pi,
$$

получаем следующее утверждение.

СлЕДСтвиЕ 1.8 (ср. [34; теорема 1]). Для любого полинома P степени $n$ u любых точек z имеет место неравенство

$$
\begin{aligned}
& \left|2 P(z) \overline{P\left(\frac{1}{\bar{z}}\right)}-M^{2}-m^{2}+2 \sqrt{\left(P(z) \overline{P\left(\frac{1}{\bar{z}}\right)}-M^{2}\right)\left(P(z) \overline{P\left(\frac{1}{\bar{z}}\right)}-m^{2}\right)}\right| \\
& \leqslant\left(M^{2}-m^{2}\right)\left|i \cos \frac{\alpha}{2} \Phi\left(i \frac{z-\cos \alpha}{\sin \alpha}\right)+\sin \frac{\alpha}{2}\right|^{n} \\
& \quad \times\left|i \cos \frac{\alpha}{2} \Phi\left(i \frac{1-z \cos \alpha}{z \sin \alpha}\right)+\sin \frac{\alpha}{2}\right|^{n},
\end{aligned}
$$

где $m=m\left(P, \Gamma_{\alpha}\right), M=M\left(P, \Gamma_{\alpha}\right), a \Phi-$ однозначная ветвъ функиии

$$
\zeta=\omega+\sqrt{\omega^{2}-1}
$$

во внешности дуги окружности, соединяющей точки \pm 1 и проходящей через точку $i \operatorname{tg}(\alpha / 2), \Phi(\infty)=\infty$. Равенство в любой точке $z$ достигается, например, для полинома

$$
P_{\alpha}(z)= \begin{cases}\prod_{k=1}^{n / 2}\left(z^{2}-2 a_{k} z+1\right) & \text { при четных } n \\ (z-1) \prod_{k=1}^{(n-1) / 2}\left(z^{2}-2 a_{k} z+1\right) & \text { при нечетнъх } n\end{cases}
$$

где $a_{k}=\cos ^{2} \frac{\alpha}{2}-\sin ^{2} \frac{\alpha}{2} \cos \frac{\pi(2 k-1)}{n}$.

ДокАЗАтЕЛьство. Неравенство следствия 1.8 вытекает из теоремы 1.16 и легко проверяемых соотношений

$$
\begin{gathered}
g_{\overline{\mathbb{C}} \backslash \Gamma_{\alpha}}(z, \infty)=\log \left|i \cos \frac{\alpha}{2} \Phi\left(i \frac{z-\cos \alpha}{\sin \alpha}\right)+\sin \frac{\alpha}{2}\right|, \\
g_{\overline{\mathbb{C}} \backslash \Gamma_{\alpha}}(z, 0)=\log \left|i \cos \frac{\alpha}{2} \Phi\left(i \frac{1-z \cos \alpha}{z \sin \alpha}\right)+\sin \frac{\alpha}{2}\right| .
\end{gathered}
$$

Докажем случай равенства. Будем считать, что $M=1$ и $m=0$. В силу теоремы 1.16 равенство достигается тогда и только тогда, когда функция $f(z)=P(z) \overline{P(1 / \bar{z})}$ осуществляет полное $2 n$-кратное накрытие области $\overline{\mathbb{C}}_{w} \backslash[0,1]$ областью $D$. Легко видеть, что в случае одной дуги такой функцией является, например, суперпозиция следующих отображений:

$$
\zeta=\frac{1}{2}\left(z+\frac{1}{z}\right), \quad u=-\frac{2 \zeta}{1-\cos \alpha}+\frac{1+\cos \alpha}{1-\cos \alpha} \quad \text { и } \quad w=\frac{1}{2}\left(T_{n}(u)+1\right) .
$$

На дуге $\Gamma_{\alpha}$ мы имеем $f(z) \equiv|P(z)|^{2}$. Нулям экстремального полинома $P$ соответствуют точки $u_{k}$ такие, что $T_{n}\left(u_{k}\right)=-1$. Если $n$ четное, скажем, $n=$ 
$2 r$, то $u_{k}=\cos (\pi(2 k-1) / n), k=1, \ldots, r$, а если $n$ нечетное, $n=2 r-1$, то $u_{k}=\cos (\pi(2 k-1) / n), k=1, \ldots, r$, и $u_{r+1}=-1$. Таким образом, корни полинома $P$ суть корни уравнений

$$
-\left(z+\frac{1}{z}\right)+2 \cos ^{2} \frac{\alpha}{2}=2 u_{k} \sin ^{2} \frac{\alpha}{2}
$$

или, что то же самое, уравнений

$$
z^{2}-2 a_{k} z+1=0
$$

где

$$
a_{k}=\left(-u_{k} \sin ^{2} \frac{\alpha}{2}+\cos ^{2} \frac{\alpha}{2}\right), \quad k=1, \ldots, r .
$$

При нечетных $n$ число $k$ принимает также значение $r+1$. Поскольку неравенство следствия 1.8 не меняется при умножении полинома $P$ на константу, то в качестве экстремального полинома можно взять, например, полином $P_{\alpha}$. Следствие доказано.

Впервые экстремальный полином из следствия 1.8 был найден Л. С. Маергойзом и Н.Н. Рыбаковой [78] в связи с решением задачи о наименьшем отклонении от нуля полиномов на дуге окружности. Соответствующие тригонометрические полиномы рассматривались ранее В. С. Виденским [81].

Теорема 1.17. Для коэфбиииентов полинома $P(z)=c_{0}+c_{1} z+\cdots+c_{n} z^{n}$, $c_{n} \neq 0$, справедливо неравенство

$$
\left|c_{n} c_{0}\right| \leqslant \frac{1}{4}\left(M^{2}-m^{2}\right) r^{2 n}(D, 0) .
$$

Равенство достигается в том и только том случае, когда функция $f(z)=$ $P(z) \overline{P(1 / \bar{z})}$ осуществляет полное 2 -кратное накрытие области $\overline{\mathbb{C}}_{w} \backslash\left[m^{2}, M^{2}\right]$ областью D.

ДоказАтельство. Из неравенства И. П. Митюка (1.26) следует, что

$$
\left|c_{n} c_{0}\right| \leqslant \frac{r^{n}(D, \infty)}{r(G, \infty)} \exp \left\{n g_{D}(0, \infty)\right\}
$$

причем равенство выполняется только в случае, указанном в теореме 1.17. Легко видеть, что

$$
\log |z|+g_{D}(z, 0) \equiv g_{D}(z, \infty)
$$

Поэтому

$$
\log r(B, 0)=g_{D}(0, \infty) .
$$

Наконец, прямые вычисления дают

$$
r\left(\overline{\mathbb{C}}_{w} \backslash\left[m^{2}, M^{2}\right], \infty\right)=\frac{4}{M^{2}-m^{2}} .
$$

Теорема доказана.

Если $c_{n}=1$ и все нули полинома $P$ расположены на дугах $\Gamma$, то $m=0$, $\left|c_{0}\right|=1$ и неравенство теоремы 1.17 имеет вид

$$
M(P, \Gamma) \geqslant 2 r^{-n}(D, 0)
$$

(cp. [77], [79; c. 177-178]). 
СлеДСтвие 1.9. Для полинома $P(z)=c_{0}+c_{1} z+\cdots+c_{n} z^{n}, c_{n} \neq 0, u \Gamma=\Gamma_{\alpha}$ имеет место точная оценка

$$
\left|c_{n} c_{0}\right| \leqslant \frac{M^{2}-m^{2}}{4 \sin ^{2 n}(\alpha / 2)} .
$$

Равенство достигается в том же случае, что и в следствии 1.8.

ДокАзАтЕльство. Требуемое неравенство вытекает из теоремы 1.17, если заметить, что

$$
r\left(\overline{\mathbb{C}}_{z} \backslash \Gamma_{\alpha}, 0\right)=r\left(\overline{\mathbb{C}}_{z} \backslash \Gamma_{\alpha}, \infty\right)=\frac{1}{\sin (\alpha / 2)}
$$

Случай равенства следует из соответствующей части следствия 1.8. Следствие доказано.

Если к тому же все нули полинома $P$ лежат на дуге $\Gamma_{\alpha}$, то следствие 1.9 эквивалентно соответствующим утверждениям А. Л. Лукашова, С. В. Тышкевича и Л. С. Маергойза, Н. Н. Рыбаковой о полиноме, наименее уклоняющемся от нуля на дуге окружности с нулями на этой дуге (ср. [77]-[79]).

Пусть $\omega(z, e, \Omega)$ обозначает гармоническую меру множества $e \subset \partial \Omega$ в точке $z$ относительно области $\Omega$. В случае $\Omega=D$ плотность гармонической меры определяется следующим образом:

$$
\widetilde{\omega}\left(\zeta, e^{i \varphi}\right)=\frac{\partial}{\partial \varphi} \omega\left(\zeta, \Gamma \cap\left\{e^{i \theta}: 0 \leqslant \theta \leqslant \varphi\right\}, D\right), \quad \zeta \in D, \quad e^{i \varphi} \in \Gamma .
$$

Теорема 1.18. Для полинома $P$ степени $n$ имеет место неравенство

$$
\left|\left(|P(z)|^{2}\right)_{\varphi}^{\prime}\right| \leqslant 2 \pi n \widetilde{\omega}(\infty, z) \sqrt{\left(M^{2}-|P(z)|^{2}\right)\left(|P(z)|^{2}-m^{2}\right)},
$$

где $z=e^{i \varphi} \in$ Г. Равенство в (1.31) достигается тогда и только тогда, когда функиия $f(z)=P(z) \overline{P(1 / \bar{z})}$ осуществляет полное 2 -кратное накрытие области $\overline{\mathbb{C}}_{w} \backslash\left[m^{2}, M^{2}\right]$ областью $D$.

ДокАЗАТЕЛЬство. Достаточно выписать неравенство (1.28) для функции $f$, указанной в теореме 1.18 , и воспользоваться известным соотношением между плотностью гармонической меры и производной по нормали от функции Грина.

В случае одной дуги плотность гармонической меры легко вычисляется и мы приходим к следующему результату.

СледСтвиЕ 1.10 (ср. [81], а также [34; теорема 2]). Для полинома P cmeneни $n$ и $z \in \Gamma_{\alpha}$ имеет место неравенство

$$
\left|\left(|P(z)|^{2}\right)_{\varphi}^{\prime}\right| \leqslant \frac{n|z+1| \sqrt{\left(M^{2}-|P(z)|^{2}\right)\left(|P(z)|^{2}-m^{2}\right)}}{\left|\sqrt{z^{2}-2 z \cos \alpha+1}\right|} .
$$

Равенство достигается в том же случае, что и в следствии 1.8.

Другие применения принципов мажорации к неравенствам для полиномов и рациональных функций представлены в работах [9], [10], [25], [30], [32], [33]. 


\section{Глава 2. Однолистные функции}

Экстремальные задачи в различных классах однолистных функций составляют наиболее изученную часть геометрической теории функций комплексного переменного (см., например, [39], [83]-[86]). В данной главе используются лишь некоторые факты этой теории, относящиеся, главным образом, к однолистным ограниченным в круге функциям. Основное внимание уделяется построению однолистных функций по заданным полиномам и описанию результатов для полиномов, вытекающих из теории однолистных функций.

2.1. Построение однолистных функций. Следующее ниже простое условие однолистности голоморфной функции появилось, по существу, в работе $[8 ; \S 2]$ (см. также [11]).

Лемма 2.1. Пусть функиия $f$ голоморфна на открытом множестве $B \subset$ $\mathbb{C}_{z}$, и пусть при приближении точки $z \kappa$ границе множества $B$ все предельные значения модуля $|f(z)|$ не менъше единицъ. Предположим, что функиия $f$ отображает некоторую точку $z_{0}$ множества $B$ и только ее в начало координат, причем $f^{\prime}\left(z_{0}\right) \neq 0$. Тогда функиия $w=f(z)$ конформно и однолистно отображает связную компоненту множества $G:=\{z \in B:|f(z)| \neq 1\}$, содержашую точку $z_{0}$, на круг $|w|<1$, а для любой другой связной компоненты $D$ множества $G$ справедливо включение $f(D) \subset\{w:|w|>1\}$.

В дальнейшем в этой главе функцию $f$, удовлетворяющую условиям леммы 2.1, будем называть допустимой. Успех в приложениях леммы 2.1 зависит от удачного выбора допустимой функции. ЖКлая показать широту применения данного условия однолистности, рассмотрим здесь несколько приемов построения допустимой функции по заданной функции из другого класса.

1. Голоморфные в круге функиии, не обращающиеся в нуль. Пусть функция $w=g(z), z \in B=U$, удовлетворяет всем условиям леммы 2.1, кроме одного она не обращается в нуль ни в одной точке круга $U$. Тогда функция

$$
f(z)=\frac{z-z_{0}}{1-\bar{z}_{0} z} g(z)
$$

является допустимой при любой фиксированной точке $z_{0}$ из круга $U$.

2. Лишние нули. Пусть функция $w=g(z), z \in B=U$, удовлетворяет всем условиям леммы 2.1, кроме одного - помимо простого нуля $z_{0}$, она имеет еще нули в точках $z_{k} \in U$ кратности $p_{k}, k=1, \ldots, n$. Тогда функция

$$
f(z)=\prod_{k=1}^{n}\left(\frac{1-\bar{z}_{k} z}{z-z_{k}}\right)^{p_{k}} g(z)
$$

допустима.

3. Ограниченные в круге функиии. Пусть функция $w=g(z)$ голоморфна в круге $U$ и $|g(z)|<1$ при $z \in U$. Предположим, что точка $z_{0} \in U$ является нулем функции $w=g(z)$ кратности $n$. Тогда функция

$$
f(z)=\left(\frac{z-z_{0}}{1-\bar{z}_{0} z}\right)^{n+1} \frac{1}{g(z)},
$$

$z \in B=\{z \in U: g(z) \neq 0\} \cup\left\{z_{0}\right\}$, допустима. Согласно лемме 2.1, она конформно и однолистно отображает связную компоненту множества $B \backslash\{z \in$ 
$U:|f(z)|=1\}$, содержащую точку $z_{0}$, на круг $|w|<1$, а любую другую связную компоненту этого множества она отображает в множество $|w|>1$.

4. Случай произвольной односвязной области гиперболического типа. Если изначально функция $w=g(z)$ задана в односвязной области $\Delta$ и если функция $z=z(\zeta)$ конформно и однолистно отображает круг $|\zeta|<1$ на область $\Delta$, то задача сводится к построению функции в круге $|\zeta|<1$ по заданной функции $\widetilde{g}(z)=g(z(\zeta)),|\zeta|<1$.

5. Функиии с вещественными граничными значениями. Пусть функция $\omega=g(z)$ мероморфна в некоторой области $\Delta$ и имеет в этой области единственный простой полюс в точке $z_{0}$. Предположим, что при приближении точки $\zeta$ к границе области $\Delta$ все предельные значения функции $g(\zeta)$ лежат на отрезке $[-1,1]$. Обозначим через

$$
\zeta=\Phi(\omega)=\omega+\sqrt{\omega^{2}-1}
$$

ту ветвь аналитической функции, обратной функции Жуковского, которая конформно и однолистно отображает внешность отрезка $[-1,1]$ на круг $|\zeta|<1$. Суперпозиция

$$
f(z)=\Phi(g(z))
$$

заданная на множестве $B=\{z \in \Delta: g(z) \notin[-1,1]\}$, допустима с данной точкой $z_{0}$.

В отличие от предыдущих пунктов этот случай требует некоторого пояснения. Во-первых, если порядок полюса $z=z_{0}$ больше единицы либо имеются другие полюсы функции $\omega=g(z)$, то $\Phi(g(z))$, конечно, не является допустимой. Однако в случае $\Delta=U$ "снятие" лишних нулей функции $\Phi(g(z))$ осуществляется с помощью приема п. 2 умножением на произведение Бляшке. В случае, когда область $\Delta$ гиперболического типа, привлекаем конформное отображение (п. 4). Функции, указанные в названии п. 5, возникают в задачах о полиномах и рациональных функциях. Например, если $R$ - рациональная функция, то функция $g(z)=R(1 / z)+\overline{R(\bar{z})}$ принимает вещественные значения на любой дуге окружности $|z|=1$ и, кроме того, возможны дальнейшие преобразования этой функции в допустимую, если имеется некоторая информация о нулях либо полюсах функции $R(z)$. Иногда вещественность функции задается априори. Многочисленные примеры использования этого приема даны в работах [8]-[10], [34]--[36]. Ниже приводятся некоторые конкретные примеры однолистных функций.

Лемма 2.2. Пусть $P$ - полином степени $n$, и пусть $L(P) \neq H(P)$. Обозначим через $\zeta=\Phi(\omega)$ функцию, конформно и однолистно отображаюшую внешность отрезка $\gamma:=[2 L(P), 2 H(P)]$ на круг $|\zeta|<1$ maк, что $\Phi(\infty)=0$, $\Phi(2 L(P))=-1$, и положим

$$
f(z):=z^{1-n} \Phi\left(P\left(\frac{1}{z}\right)+\overline{P(\bar{z})}\right) .
$$

Тогда множество $G:=\{z:|z|<1, P(1 / z)+\overline{P(\bar{z})} \notin \gamma,|f(z)| \neq 1\}$ состоuт из конечного числа областей $\{D\}$, ограниченных конечным числом кусочно гладких кривых и обладающих следующими свойствами. Если D из совокупности $\{D\}$ не содержит начала координат, то образ области $D$ при отображении $w=f(z)$ принадлежит множеству $|w|>1$. Если область $D$ содержит 
точку $z=0$, то функиия $f$ конформно и однолистно отображает область $D$ на круг $|w|<1$.

ДокАЗАтЕльство. Достаточно проверить, что функция $f$ является допустимой на множестве $B=\{z:|z|<1, P(1 / z)+\overline{P(\bar{z})} \notin \gamma\}$, и применить леммy 2.1 с $z_{0}=0$.

ЗАмечАниЕ 2.1. Утверждения леммы 2.2 остаются в силе, если полином $P$ заменить на рациональную функцию $R(z)=z^{-k} P(z)$, где $k$ - целое неотрицательное число, а $P$ - многочлен степени $n$. В этом случае необходимо также заменить степень $1-n$ переменной $z$ в определении функции $f$ на $1+m$, где $m=k-n$, если $k<n / 2$, и $m=-k$ в противном случае.

Аналогично доказываются следующие леммы 2.3-2.5.

Лемма 2.3. Пусть $P(z)=c_{0}+c_{1} z+\cdots+c_{n} z^{n}, \operatorname{Re} c_{n} \neq 0$, u nyсm $\bar{L}(P) \neq$ $\bar{H}(P)$. Обозначим через $\zeta=\Phi(\omega)$ функиию, конформно и однолистно отображаюшую внешность отрезка $\bar{\gamma}:=[2 \bar{L}(P), 2 \bar{H}(P)]$ на круг $|\zeta|<1$ так, что $\Phi(\infty)=0, \Phi(2 \bar{L}(P))=-1$, и положим

$$
f(z):=z^{1-n} \Phi\left(P\left(\frac{z+1 / z}{2}\right)+\overline{P\left(\frac{\bar{z}+1 / \bar{z}}{2}\right)}\right) .
$$

Тогда для множества

$$
G:=\left\{z:|z|<1, P\left(\frac{z+1 / z}{2}\right)+\overline{P\left(\frac{\bar{z}+1 / \bar{z}}{2}\right)} \notin \bar{\gamma},|f(z)| \neq 1\right\}
$$

и функиии $f$ справедливы утверждения леммы 2.2 .

Заметим, что на самом деле условие $\bar{L}(P) \neq \bar{H}(P)$ для данного полинома $P$ степени $n \geqslant 1$ всегда выполняется. Это же касается условия $L(P) \neq H(P)$ в лемме 2.2.

Лемма 2.4. Предположим, что все нули полинома $P(z)=c_{0}+c_{1} z+\cdots+$ $c_{n} z^{n}, c_{0} \neq 0, c_{n} \neq 0$, лежат в круге $U$, и пусть

$$
f(z):=z^{n+1} \frac{P(1 / z)}{\overline{P(\bar{z})}} .
$$

Тогда множество

$$
G:=\{z:|z|<1, f(z) \neq \infty,|f(z)| \neq 1\}
$$

cостоит из конечного числа областей $\{D\}$, ограниченных конечным числом кусочно гладких кривых (возможно, вырожденных), причем если точка $z=0$ принадлежит области $D$ из совокупности $\{D\}$, то функиия $w=f(z)$ отображает эту область конформно и однолистно на круг $|w|<1$, а если $z=0$ не принадлежит $D$, то образ $f(D)$ лежит вне этого круга.

Лемма 2.5. Предположим, что все нули полинома $P(z)=c_{0}+c_{1} z+\cdots+$ $c_{n} z^{n}, c_{0} \neq 0, c_{n} \neq 0$, лежат в множестве $|z|>1$, и пусть

$$
f(z):=z^{1-n} \frac{\overline{P(\bar{z})}}{P(1 / z)}
$$

Тогда множество $G:=\{z:|z|<1, f(z) \neq \infty,|f(z)| \neq 1\}$ обладает свойством, указанным в лемме 2.4 . 
Лемма 2.6. Пусть $G$-односвязная область, содержащая точку $z=\infty, u$ пусть функиия $z=\Psi(w)$ конформно и однолистно отображает круг $|w|<1$ на область $G$ так, что $\Psi(0)=\infty$. Предположим, что все нули полинома $P$ степени $n$ лежат в дополнении $G$, и пусть $M:=\max \{|P(z)|: z \in \partial G\}$. Тогда функиия

$$
z=F(w) \equiv\left(\frac{P(\Psi(w))}{M}\right)^{-1 / n}, \quad|w|<1,
$$

распадается в круге $|w|<1$ на $n$ регулярных ветвей, каждая из которьх однолистно покрывает круг $|z|<1$.

ДокАЗАТЕЛЬство. Регулярность ветвей следует из теоремы о монодромии, а однолистность накрытия - из леммы 2.1.

Лемма 2.7. Пусть $P$ - полином степени $n$, и пусть все его конечнъе критические значения лежат в дополнении $\kappa$ некоторой односвязной области $G$ гиперболического типа, содержащей бесконечно удаленную точку. Обозначим через $\zeta=\Phi(w)$ функиию, конформно и однолистно отображающую область $G$ на круг $|\zeta|<1$ так, что $\Phi(\infty)=0, \lim _{\omega \rightarrow \infty} \omega \Phi(\omega)>0$, и положим

$$
\zeta=Q(z) \equiv(\Phi(P(z)))^{1 / n}, \quad z \in B:=\{z: P(z) \in G\} .
$$

Тогда функиия $\zeta=Q(z)$ распадается в односвязной области $B$ на п регулярных ветвей, каждая из которых однолистно отображает эту область на круг $|\zeta|<1$.

ДокАЗАтельство. Полином $P$ взаимно однозначно отображает $z$-сферу на некоторую риманову поверхность $\mathscr{R}$, лежащую над $w$-сферой. Часть поверхности $\mathscr{R}$, лежащая над $G$, с единственной точкой ветвления в бесконечности, гомеоморфно отображается посредством функции $\zeta=\Phi(w)$ на $n$-листный единичный круг. Ветви функции $\sqrt[n]{\zeta}$ разворачивают этот круг на круг $|\zeta|<1$. Таким образом, суперпозиция $\zeta=Q(z)$ конформно и однолистно отображает область $B$ на круг $|\zeta|<1$. Лемма доказана.

2.2. Теоремы покрытия для полиномов. Термин "теорема покрытия" хорошо известен в геометрической теории функций [39]. Применительно к полиномам $P(z)$ под такой теоремой мы понимаем информацию о геометрии образа заданного множества при отображении $w=P(z)$ либо функциями от $P(z)$. В данном разделе приводятся теоремы из работы [8] о покрытии функциями вида $P(z)+\overline{P(1 / \bar{z})}, P(z)+\overline{P(\bar{z})}, P(z) \overline{P(1 / \bar{z})}$ и $P(z) / \overline{P(1 / \bar{z})}$. Всюду ниже приняты обозначения из введения. Положим также

$$
M(P, r):=\max \{|P(z)|:|z|=r\}, \quad M(P, 1)=M(P) .
$$

Tеорема 2.1. Пусть $P(z)=c_{0}+c_{1} z+\cdots+c_{n} z^{n}, c_{n} \neq 0$. Тогда образ окружности $|z|=r$ при отображении $\omega=P(z)+\overline{P(1 / \bar{z})}$ в случае $r=1$ есть отрезок вещественной оси длинь

$$
2 H(P)-2 L(P) \geqslant 4\left|c_{n}\right|,
$$

а при $r>1$ этот образ есть кривал, лежащая внутри эллипса с фокусами в точках $2 L(P)$ и $2 H(P)$ и большой осъю, равной

$$
\left(r^{1-n} x_{0}+\frac{r^{n-1}}{x_{0}}\right)(H(P)-L(P)) \leqslant\left(r^{-n}+r^{n}\right)(H(P)-L(P)),
$$


где $x_{0}-$ коренъ уравнения

$$
2\left|c_{n}\right|(1-r)^{2} x=r(H(P)-L(P))(1-x)^{2},
$$

принадлежащий промежутку $1 / r \leqslant x<1$. Для полиномов вида $P(z)=c_{0}+$ $c_{n} z^{n}$ выполняются равенства в (2.1) и (2.2) и образом окружности $|z|=r$, $r>1$, явлется указанный выше эллипс.

ДоказАтельство. Можно считать, что $H(P) \neq L(P)$. Обозначим через $z=F(w)$ функцию, заданную в круге $|w|<1$ и обратную к функции $w=f(z)$ из леммы 2.2. Так как в круге $|w|<1$ имеет место разложение

$$
F(w)=\left[\frac{2 c_{n}}{H(P)-L(P)}\right] w+\cdots,
$$

то лемма Шварца дает неравенство (2.1). Возьмем произвольную точку $z$ на окружности $|z|=1 / r, r>1$. Имеем либо $|f(z)| \geqslant 1$, либо $|f(z)|<1$. В первом случае выполняется

$$
\left|\Phi\left(P\left(\frac{1}{z}\right)+\overline{P(\bar{z})}\right)\right| \geqslant|z|^{n-1}=\left(\frac{1}{r}\right)^{n-1} .
$$

Во втором по лемме 2.2 заключаем, что точка $z$ является образом некоторой точки $w$ при отображении функцией $F$. Из теоремы Пика [84; с. 55] следует, что

$$
\frac{|w|}{(1-|w|)^{2}} \geqslant \frac{r}{\left|f^{\prime}(0)\right|(1-r)^{2}} \geqslant \frac{r}{(1-r)^{2}}=\frac{1 / r}{(1-1 / r)^{2}},
$$

а это равносильно неравенству

$$
|w| \geqslant x_{0} \geqslant \frac{1}{r}
$$

где $x_{0}$ - корень уравнения $(2.3), 1 / r \leqslant x_{0}<1$. Отсюда вытекает, что

$$
\left|\Phi\left(P\left(\frac{1}{z}\right)+\overline{P(\bar{z})}\right)\right| \geqslant x_{0}\left(\frac{1}{r}\right)^{n-1} \geqslant\left(\frac{1}{r}\right)^{n} .
$$

Сравнивая (2.4) и (2.5), получаем, что для любых точек $z$ на окружности $|z|=1 / r$ справедливо неравенство (2.5). Следовательно, точка $P(1 / z)+\overline{P(\bar{z})}$ или, что то же самое, точка $P(z)+\overline{P(1 / \bar{z})}$ при $|z|=r$ лежит внутри эллипса, указанного в теореме, и имеет место неравенство (2.2). Утверждение об экстремальном полиноме $c_{0}+c_{n} z^{n}$ проверяется непосредственным вычислением. Теорема доказана.

Неравенство (2.1) восходит к Сегё [87].

Tеорема 2.2. Пусть $P(z)=c_{0}+c_{1} z+\cdots+c_{n} z^{n}$, Re $c_{n} \neq 0$. Тогда образ "эллипса" $|z-1|+|z+1|=r+1 / r$ при отображении $\omega=P(z)+\overline{P(\bar{z})}$ в случае $r=1$ есть отрезок вещественной оси длинь

$$
2 \bar{H}(P)-2 \bar{L}(P) \geqslant 2^{3-n}\left|\operatorname{Re} c_{n}\right|,
$$

а при $r>1$ этот образ есть кривая, лежащая внутри эллипса с фокусами в точках $2 \bar{L}(P)$ и $2 \bar{H}(P)$ и большой осъю, равной

$$
\left(r^{1-n} x_{0}+\frac{r^{n-1}}{x_{0}}\right)(\bar{H}(P)-\bar{L}(P)) \leqslant\left(r^{-n}+r^{n}\right)(\bar{H}(P)-\bar{L}(P)),
$$


где $x_{0}-$ корень уравнения

$$
\left|\operatorname{Re} c_{n}\right|(1-r)^{2} x=r \cdot 2^{n-2}(\bar{H}(P)-\bar{L}(P))(1-x)^{2}
$$

на промежутке $1 / r \leqslant x<1$. Равенство в (2.6) и (2.7) достигается для полинома Чебышёва $T_{n}(z)=2^{n-1} z^{n}+\cdots$, который отображает эллипс $|z-1|+|z+1|=r+1 / r$ на эллипс $|w-1|+|w+1|=r^{n}+1 / r^{n}, r>1$.

ДокАЗАтельство. Можно считать, что $\bar{H}(P) \neq \bar{L}(P)$. Повторяя доказательство теоремы 2.1, но с функцией $f$ из леммы 2.3, получим, согласно (1.12), неравенство (2.6). Далее, как и выше с $|z|=1 / r, r>1$, применяя теорему Пика, приходим к неравенству

$$
\left|\Phi\left(P\left(\frac{z+1 / z}{2}\right)+\overline{P\left(\frac{\bar{z}+1 / \bar{z}}{2}\right)}\right)\right| \geqslant x_{0}\left(\frac{1}{r}\right)^{n-1} \geqslant\left(\frac{1}{r}\right)^{n},
$$

аналогичному (2.5). Здесь $\zeta=\Phi(\omega)$ - из леммы 2.3 , а $x_{0}$ - корень уравнения $(2.8), 1 / r \leqslant x_{0}<1$. Отсюда следует утверждение теоремы 2.2 о принадлежности точки $P(z)+\overline{P(\bar{z})}$ внутренности соответствующего эллипса. Случай равенства выводится непосредственно из определения полинома Чебышёва. Теорема доказана.

Неравенство (2.6) совпадает с классическим свойством чебышёвских полиномов наименее отклоняться от нуля. Напомним, что мы получили его из неравенства Шварца. Теорема 2.2 о покрытии при $r$, близких к единице, приводит к неравенству бернштейновского типа для производной полинома на отрезке. Замечая, что для полинома $P$ с вещественными коэффициентами выполняется $\overline{P(\bar{z})} \equiv P(z)$, получаем из теоремы 2.2 результат К. Дочева [40] и, в частности, неравенство Чебышёва

$$
|P(x)| \leqslant\left|T_{n}(x)\right| \bar{M}(P), \quad x \in \mathbb{R} \backslash[-1,1]
$$

(см. теорему 1.1 и следующий за ней комментарий). ЖКелая уточнить неравенство Чебышёва для полиномов с критическими точками на отрезке $[-1,1]$, введем сперва обозначение

$$
\delta(P):=\sqrt[n]{\frac{2^{2-n}\left|\operatorname{Re} c_{n}\right|}{\bar{H}(P)-\bar{L}(P)}},
$$

где $P(z)=c_{0}+c_{1} z+\cdots+c_{n} z^{n}, c_{k} \in \mathbb{C}, k=0,1, \ldots, n, \operatorname{Re} c_{n} \neq 0$. Согласно (2.6), для любых полиномов $P$ выполняется $\delta(P) \leqslant 1$ с равенством в случае $P(z)=$ $a T_{n}(z)+b, a \in \mathbb{R} \backslash\{0\}, b \in \mathbb{C}$.

Теорема 2.3. Предположим, что все критические точки полинома $P$ степени $n$ с вещественными коэффичиентами расположены на отрезке $[-1,1]$. Тогда для любых вещественнъх $x,|x| \geqslant 1$, справедливы неравенства

$$
\frac{2|P(x)-(\bar{L}(P)+\bar{H}(P)) / 2|}{\bar{H}(P)-\bar{L}(P)} \leqslant T_{n}((|x|-1) \delta(P)+1) \leqslant T_{n}(|x|),
$$

а для $|x| \geqslant(2-\delta(P)) / \delta(P)$ имеет место также неравенство в другую сторону

$$
\frac{2|P(x)-(\bar{L}(P)+\bar{H}(P)) / 2|}{\bar{H}(P)-\bar{L}(P)} \geqslant T_{n}((|x|+1) \delta(P)-1) .
$$


ДокАЗАТЕЛЬство этой теоремы вновь опирается на теорему Пика, однако в этом случае необходимо использовать лемму 2.7 (см. [8; теорема 3.3]).

Tеорема 2.4. Пусть $P(z)=c_{0}+c_{1} z+\cdots+c_{n} z^{n}, c_{0} \neq 0, c_{n} \neq 0$. Тогда образ окружности $|z|=r$ при отображении $\omega=P(z) \overline{P(1 / \bar{z})}$ в случае $r=1$ есть отрезок вещественной оси длинъ

$$
M^{2}(P)-m^{2}(P) \geqslant 4\left|c_{0} c_{n}\right|,
$$

а при $r>1$ этот образ есть кривая, лежащая внутри эллипса с фокусами в точках $m^{2}(P)$ и $M^{2}(P)$ и болъшой осъю, равной

$$
\frac{1}{2}\left(r^{1-n} x_{0}+\frac{r^{n-1}}{x_{0}}\right)\left(M^{2}(P)-m^{2}(P)\right) \leqslant \frac{1}{2}\left(r^{-n}+r^{n}\right)\left(M^{2}(P)-m^{2}(P)\right),
$$

где $x_{0}-$ корень уравнения

$$
4\left|c_{0} c_{n}\right|(1-r)^{2} x=r\left(M^{2}(P)-m^{2}(P)\right)(1-x)^{2},
$$

принадлежащий промежутку $0<x<1$. Равенства выполняются для полиномов вида $P(z)=c_{0}+c_{n} z^{n},\left|c_{0}\right|=\left|c_{n}\right| \neq 0$, при которых соответствующий образ окружности $|z|=r, r>1$, есть указанный выше эллипс.

ДокАЗАТЕльство аналогично доказательству теоремы 2.1, но с привлечением вместо функции из леммы 2.2 функции

$$
f(z)=z^{1-n} \Phi\left(P\left(\frac{1}{z}\right) \overline{P(\bar{z})}\right)
$$

где $\zeta=\Phi(\omega)$ есть конформное и однолистное отображение внешности отрезка $\left[m^{2}(P), M^{2}(P)\right]$ на круг $|\zeta|<1$ такое, что $\Phi(\infty)=0$ и $\Phi\left(m^{2}(P)\right)=-1$.

С этого момента читатель без труда получит аналог теоремы 2.2 , но уже для отображения $\omega=P(z) \overline{P(\bar{z})}$, а также утверждение о покрытии функцией $P^{2}(z)$, аналогичное теореме 1.1 , для полиномов $P(z)$ с вещественными коэффициентами.

При доказательстве полиномиальных неравенств зачастую полезно переходить от заданного полинома $P(z)$ к полиномам $z^{n} \overline{P(1 / \bar{z})}$ либо $z^{n} P(1 / z)$ (см., например, [2], [88], [89]). Отметим, что полиномы, для которых $\bar{c}_{n} P(z) \equiv$ $c_{0} z^{n} \overline{P(1 / \bar{z})}$, характеризуются тем, что множество их корней симметрично относительно окружности $|z|=1$. Такие полиномы, а также полиномы вида $P(z) \equiv z^{n} P(1 / z)$ изучались отдельно [90]-[92]. Для них теоремы покрытия настоящего раздела приобретают особое звучание. Например, из теоремы 2.4 вытекает следующий факт.

СлеДСТвИЕ 2.1. Если корни полинома $P$ степени $n$ симметричны относительно окружности $|z|=1$, то для любого $r, 0<r \leqslant 1$, выполняется неравенство

$$
M^{2}(P, r) \leqslant r^{n}\left[\left(r^{n-1} x_{0}+r^{1-n} x_{0}^{-1}\right) \frac{M^{2}(P)-m^{2}(P)}{4}+\frac{M^{2}(P)+m^{2}(P)}{2}\right],
$$

где $x_{0}$ из теоремы $2.4, r \leqslant x_{0} \leqslant 1$. Равенство достигается в случае $P(z)=$ $c_{0}+c_{n} z^{n},\left|c_{0}\right|=\left|c_{n}\right| \neq 0$. 
Заменяя в (2.10) $x_{0}$ на $r$, легко заключаем, что в условиях следствия 2.1 справедливо неравенство

$$
M(P, r) \leqslant \frac{1+r^{n}}{2} M(P, 1), \quad r \leqslant 1 .
$$

Последнее неравенство справедливо также для $r>1$, в чем убеждаемся заменой $P(z)$ на $z^{n} \overline{P(1 / \bar{z})}$.

Следующий результат вытекает вновь из теоремы Пика, но с применением функции из леммы 2.4 .

Теорема 2.5 (см. теорему 1.4). Если все нули полинома $P(z)=c_{0}+c_{1} z+$ $\cdots+c_{n} z^{n}, c_{0} \neq 0, c_{n} \neq 0$, лежат в круге $|z| \leqslant 1$, то для любого $r>1$ и любой точки z на окружности $|z|=r$ выполняется неравенство

$$
\left|\frac{P(1 / \bar{z})}{P(z)}\right| \leqslant x^{*} r^{-n-1} \leqslant r^{-n}
$$

где $x^{*}>1$ - коренъ уравнения

$$
\left|c_{0}\right|(1-r)^{2} x=r\left|c_{n}\right|(1-x)^{2} .
$$

Равенство достигается для полиномов $P(z)$ с нулями на единичной окружности $|z|=1$.

Неравенство (2.11) можно интерпретировать как оценку произведения Бляшке

$$
\left|\prod_{k=1}^{n} \frac{1-\bar{a}_{k} z}{z-a_{k}}\right| \leqslant \frac{x^{*}}{|z|} \leqslant 1
$$

при $|z|>1$ и $\left|a_{k}\right| \leqslant 1, k=1, \ldots, n$. Здесь “итоговое" неравенство есть очевидное следствие свойства дробно-линейного отображения.

Теорема 2.6. Если полином $P(z)=c_{0}+c_{1} z+\cdots+c_{n} z^{n}, c_{n} \neq 0$, не имеет нулей в круге $|z|<1$, то для любого $r>1$ и любой точки $z$ на окружности $|z|=r$ выполняется неравенство

$$
\left|\frac{P(1 / \bar{z})}{P(z)}\right| \geqslant x_{*} r^{1-n} \geqslant r^{-n},
$$

где $x_{*}, 0<x_{*}<1,-$ корень уравнения

$$
\left|c_{n}\right|(1-r)^{2} x=r\left|c_{0}\right|(1-x)^{2} .
$$

Равенство достигается для полиномов $P(z)$ с нулями на единичной окружности $|z|=1$.

ДокАЗАтЕльство. Достаточно применить предыдущую теорему к полиному $z^{n} P(1 / z)$ либо повторить доказательство этой теоремы с заменой леммы 2.4 на лемму 2.5 .

СлЕДСТВИЕ 2.2. В условиях и обозначениях теоремы 2.6 справедливо неравенство

$$
M(P, r) \leqslant \frac{1+r^{n}}{1+x_{*} r} M(P, 1)
$$

с равенством для полиномов $P(z)=c_{0}+c_{n} z^{n},\left|c_{0}\right|=\left|c_{n}\right| \neq 0$. 
ДоказАТеЛЬство. Известно, что для полиномов $P$ степени $n$ с $M(P, 1)=1$ на окружности $|z|=r$ выполняется неравенство

$$
|P(z)|+r^{n}\left|P\left(\frac{1}{\bar{z}}\right)\right| \leqslant r^{n}+1
$$

(см. [58; с. 550]). Осталось применить теорему 2.6 и перейти к максимуму.

Заменяя в данном следствии $x_{*} r$ на единицу, вновь получаем неравенство Н. Энкени и Т. Ривлина [59]. Другое усиление этого неравенства можно получить, комбинируя теоремы 2.4 и 2.5 .

2.3. Неравенства с участием производных. Теоремы теории однолистных функций в сочетании с леммами из раздела 2.1 либо их модификациями дают целый ряд неравенств с участием производных от полиномов. Ниже приводятся типичные результаты с небольшими комментариями.

Теорема 2.7. Пусть $P(z)=c_{0}+c_{1} z+\cdots+c_{n} z^{n}$, u пусть $k$ - иелое неотрицательное число. Тогда для любых точек z на окружности $|z|=1$ выполняются неравенства

$$
\begin{array}{ll}
\left|z P^{\prime}(z)-k P(z)\right| \leqslant(n-k-1) M(P)+\sqrt{\left|c_{n}\right| M(P)}, & \text { если } k<\frac{n}{2}, \\
\left|z P^{\prime}(z)-k P(z)\right| \leqslant\left(\frac{n}{2}-1\right) M(P)+\sqrt{\left(\left|c_{n}\right|+\left|c_{0}\right|\right) M(P)}, & \text { если } k=\frac{n}{2}, \\
\left|z P^{\prime}(z)-k P(z)\right| \leqslant(k-1) M(P)+\sqrt{\left|c_{0}\right| M(P)}, & \text { если } k>\frac{n}{2} .
\end{array}
$$

Равенство в первом и во втором случаях достигается, например, при $P(z)=$ $c_{n} z^{n}$, а в третъем неравенстве - при $P(z)=c_{0}$.

ДокАЗАТЕЛЬство. Случаи равенства проверяются непосредственно. При доказательстве неравенств можно считать, что $c_{0} \neq 0, c_{n} \neq 0,\left|c_{n}+\bar{c}_{0}\right| \neq 0$, а точки $z$ не принимают некоторое конечное число значений на окружности $|z|=1$. Введем следующие обозначения:

$R(z)=z^{-k} P(z)$

$\zeta=\Phi(\omega)$ - конформное и однолистное отображение внешности отрезка $\gamma:=$ $[2 L(R), 2 H(R)]$ на круг $|\zeta|<1$ такое, что $\Phi(\infty)=0, \Phi(2 L(R))=-1$;

$m=k-n$, если $k<n / 2$, и $m=-k-$ в противном случае;

$$
f(z)=z^{m+1} \Phi\left(R\left(\frac{1}{z}\right)+\overline{R(\bar{z})}\right), \quad|z|<1, \quad R\left(\frac{1}{z}\right)+\overline{R(\bar{z})} \notin \gamma .
$$

Согласно замечанию 2.1, множество $\{z:|z|<1, R(1 / z)+\overline{R(\bar{z})} \notin \gamma,|f(z)| \neq 1\}$ состоит из конечного числа областей $\{D\}$ с кусочно гладкими границами, причем если область $D$ не содержит начало, то $f(D)$ лежит вне единичного круга, а если $0 \in D$, то $w=f(z)$ конформно и однолистно отображает область $D$ на круг $U_{w}$. Если теперь $1 / z$ - регулярная для $f$ точка окружности $|z|=1$ и в то же время $f(D)$ лежит вне круга $U_{w}$, то необходимо в этой точке

$$
\frac{\partial|f|}{\partial|z|} \leqslant 0
$$


В противном случае неравенство (1.12) (примененное к обратной функции) дает

$$
\frac{\partial|f|}{\partial|z|} \leqslant \sqrt{\frac{1}{\left|f^{\prime}(0)\right|}} \leqslant 1
$$

где

$$
\frac{1}{\left|f^{\prime}(0)\right|}=\left\{\begin{array}{lll}
\frac{2\left|c_{n}\right|}{H(R)-L(R)}, & \text { если } & k<n / 2, \\
\frac{2\left|c_{n}+\bar{c}_{0}\right|}{H(R)-L(R)}, & \text { если } & k=n / 2, \\
\frac{2\left|c_{0}\right|}{H(R)-L(R)}, & \text { если } & k>n / 2 .
\end{array}\right.
$$

Сравнивая (2.12) и (2.13), заключаем, что всегда выполняется (2.13). Для вычисления производной слева представим функцию $\zeta=\Phi(\omega)$ в виде суперпозиции

$$
\zeta=\eta+\sqrt{\eta^{2}-1}, \quad \eta=\frac{\omega-H(R)-L(R)}{H(R)-L(R)},
$$

где ветвь корня выбрана подходящим образом. Заметим, что при $|z|=1$ точка $\omega=R(1 / z)+\overline{R(\bar{z})}$ принадлежит $\gamma$ и $\eta:=u+i v \in[-1,1]$. Поэтому

$$
\left|\zeta^{\prime}(\eta)\right|=\left|1 \pm \frac{u i}{\sqrt{1-u^{2}}}\right|=\frac{1}{\sqrt{(1-u)(1+u)}}, \quad-1<u<1 .
$$

Учитывая последнее замечание, имеем

$$
\frac{\partial|f|}{\partial|z|}=m+1+\frac{\left|\operatorname{Im}\left(z R^{\prime}(z)\right)\right|}{\sqrt{(\operatorname{Re} R(z)-L(R))(H(R)-\operatorname{Re} R(z))}} \geqslant m+1+\frac{2\left|\operatorname{Im}\left(z R^{\prime}(z)\right)\right|}{H(R)-L(R)} .
$$

Пусть для определенности $k<n / 2$. Тогда из неравенства (2.13) следует, что

$$
\begin{aligned}
\left|\operatorname{Im}\left(z R^{\prime}(z)\right)\right| & \leqslant \frac{1}{2}(n-k-1)(H(R)-L(R))+\frac{1}{2} \sqrt{\left|c_{n}\right|(H(R)-L(R))} \\
& \leqslant(n-k-1) M(P)+\sqrt{\left|c_{n}\right| M(P)} .
\end{aligned}
$$

Заменяя при необходимости $P(z)$ на $e^{i \theta} P(z)$ с подходящим $\theta$, имеем $\left|R^{\prime}(z)\right|$ в левой части последнего неравенства, и, следовательно, утверждение теоремы 2.7 доказано при $k<n / 2$. Аналогично рассматриваются случаи $k=n / 2$ и $k>n / 2$. Теорема доказана.

Отметим, что промежуточное неравенство в доказательстве теоремы 2.7 при $k=0$ восходит к Сегё:

$$
\left|P^{\prime}(z)\right| \leqslant n \max \{|\operatorname{Re} P(z)|:|z|=1\}, \quad|z|=1
$$

(см. также [93]). Итоговое неравенство для этого же значения $k$ усиливает классическое неравенство Бернштейна:

$$
\left|P^{\prime}(z)\right| \leqslant n M(P), \quad|z|=1 .
$$

Желая иметь в правой части первой оценки теоремы 2.7 только геометрические характеристики, воспользуемся известным неравенством $S \geqslant \pi\left|c_{n}\right|^{2}$ для 
площади $S$ образа круга $U$ при отображении $w=P(z)$ [94; отдел 3 , задача 124]. Указанная оценка дает

$$
\left|z P^{\prime}(z)-k P(z)\right| \leqslant(n-k-1) R+\sqrt{R \sqrt{\frac{S}{\pi}}}, \quad k<\frac{n}{2},
$$

где $z$ - произвольная точка круга $U, R$ - радиус круга с центром в начале, содержащего множество $P(U)$, а $S$ - площадь $P(U)$ без учета листности накрытия. Равенство достигается в точках $z,|z|=1$, для полинома $P(z)=c_{n} z^{n}$ и любого целого $k, 0 \leqslant k<n / 2$.

Ввиду (2.13) при четном $n(k=n / 2)$ имеем уточнение следующего неравенства В. И. Смирнова [95]:

$$
\left|z P^{\prime}(z)-\frac{n}{2} P(z)\right| \leqslant \frac{n}{2} M(P)
$$

(см. также [96]). Из более поздних результатов В. И. Смирнова [97] вытекает неравенство

$$
\left|z P^{\prime}(z)-\alpha P(z)\right| \leqslant|n-\alpha| M(P),
$$

справедливое для всех точек $z,|z|=1$, и комплексных чисел $\alpha, \operatorname{Re} \alpha \leqslant n / 2$. Теорема 2.7 дает уточнения этого неравенства в случае любых вещественных $\alpha$ (см. [10; теоремы 4, 5]).

Заменяя модуль в левой части неравенств теоремы 2.7 на разность модулей, легко приходим к следующему утверждению.

СЛЕДСТВИЕ 2.3. Для любого полинома $P(z)=c_{0}+c_{1} z+\cdots+c_{n} z^{n}$ четной степени $n \geqslant 2$ справедливы оченки

$$
\begin{aligned}
0 & \leqslant M(P)-\min \left(\sqrt{\left|c_{0}\right| M(P)}, \sqrt{\left(\left|c_{0}\right|+\left|c_{n}\right|\right) M(P)}\right) \leqslant M\left(P^{\prime}\right) \\
& \leqslant(n-1) M(P)+\min \left(\sqrt{\left(\left|c_{0}\right|+\left|c_{n}\right|\right) M(P)}, \sqrt{\left|c_{n}\right| M(P)}\right)
\end{aligned}
$$

с равенством, например, при $P(z)=c_{0}$ для нижней оценки и $P(z)=c_{n} z^{n}$ для верхней.

Следующее утверждение содержит классическое неравенство Бернштейна для полиномов на отрезке.

Теорема 2.8. Для любого полинома $P(z)=c_{0}+c_{1} z+\cdots+c_{n} z^{n}, \operatorname{Re} c_{n} \neq 0$, и любой точки $x$ на отрезке $[-1,1]$ справедливо неравенство

$$
\left|\operatorname{Re} P^{\prime}(x)\right| \sqrt{1-x^{2}} \leqslant\left(n-1+\sqrt{\delta^{n}(P)}\right) \sqrt{(\operatorname{Re} P(x)-\bar{L}(P))(\bar{H}(P)-\operatorname{Re} P(x))} .
$$

Равенство в (2.14) достигается в случае $P(z)=a T_{n}(z)+b$ для любих вещественных $a \neq 0$, комплексных $b$ u $x \in[-1,1]$.

ДокАзАтельство. Величина $\delta(P)$ определена равенством (2.9). Повторим схему доказательства теоремы 2.7, но с другими вспомогательными функциями. Именно, пусть функции $\zeta=\Phi(\omega)$ и $w=f(z)$ из леммы 2.3. Как и выше, лемма 2.3 и неравенство (1.12) дают в точках регулярности $1 / z$ на окружности $|z|=1$ неравенство

$$
\frac{\partial|f|}{\partial|z|} \leqslant(\delta(P))^{n / 2} \leqslant 1
$$


(ср. (2.13)). Осталось подсчитать производную в левой части неравенства (2.15), что с учетом вычислений в доказательстве теоремы 2.7 является простым упражнением. Случай равенства проверяется непосредственно в (2.14) либо в (2.15). Теорема доказана.

Следующая цепочка неравенств с учетом (2.15) показывает, что (2.14) действительно является усилением неравенства Бернштейна [2; с. 233]:

$$
\begin{aligned}
\left|\operatorname{Re} P^{\prime}(z)\right| \sqrt{1-x^{2}} & \leqslant n \sqrt{(\operatorname{Re} P(x)-\bar{L}(P))(\bar{H}(P)-\operatorname{Re} P(x))} \\
& \leqslant \frac{n}{2}(\bar{H}(P)-\bar{L}(P)) \leqslant n \bar{M}(P) .
\end{aligned}
$$

Аналогично, из (2.14) получаем для $x \in[-1,1]$ неравенство

$$
\left|P^{\prime}(x)\right| \sqrt{1-x^{2}} \leqslant(n-1) \bar{M}(P)+\sqrt{2^{1-n}\left|\operatorname{Re} c_{n}\right| \bar{M}(P)} \leqslant n \bar{M}(P) .
$$

Отсюда вытекает также известное неравенство Маркова:

$$
\left|P^{\prime}(x)\right| \leqslant n^{2} \bar{M}(P), \quad-1 \leqslant x \leqslant 1 .
$$

Заменяя в доказательстве теоремы 2.8 неравенство (1.12) на неравенство Н. А. Лебедева [98; с. 9, 12]):

$$
\left|f^{\prime}(z)\right| \geqslant \exp \left(\left|\arg \frac{f(z)}{z}\right|\right)
$$

приходим к следующему результату [23; теорема 2].

Теорема 2.9. Для полинома $P$ степени $n$ с вещественными коэффициентами, удовлетворяющего условию $M(P) \leqslant 1$, справедливо неравенство

$$
\frac{\left|P^{\prime}(x)\right| \sqrt{1-x^{2}}}{\sqrt{1-P^{2}(x)}} \leqslant n-1+\exp \left(-\left|\arccos P(x)-\arccos T_{n}(x)\right|\right),
$$

с равенством при любых $x \in(-1,1)$ для полинома Чебышёва $T_{n}$.

Доказательства следующих ниже теорем 2.10-2.14 опираются на неравенство (1.12). Отличия заключаются только в выборе функций, к которым оно применяется (точнее, обратных функций). Например, функция из леммы 2.7 приводит к следующему утверждению [8].

Теорема 2.10. Для любого полинома $P(z)=c_{0}+c_{1} z+\cdots+c_{n} z^{n}$ с коэффичиентами $c_{k} \in \mathbb{R}, k=0,1, \ldots, n, c_{n} \neq 0$, и с критическими точками на отрезке $[-1,1]$ выполняется неравенство

$$
\left|P^{\prime}(x)\right| \sqrt{1-x^{2}} \leqslant n \sqrt{\delta(P)} \sqrt{(P(x)-\bar{L}(P))(\bar{H}(P)-P(x))}, \quad x \in[-1,1] .
$$

Равенство достигается для полиномов $a T_{n}(z)+b, a \in \mathbb{R} \backslash\{0\}, b \in \mathbb{R}$.

СлеДСтвиЕ 2.4. В условиях предъдущей теоремы имеем

$$
\left|P^{\prime}(x)\right| \leqslant n^{2} \bar{M}(P)\left|\frac{2^{1-n} c_{n}}{\bar{M}(P)}\right|^{1 / n}, \quad-1 \leqslant x \leqslant 1,
$$

с равенством для полиномов Чебышёва $T_{n}$. 
Функция из леммы 2.2 дает следующую теорему (см. [7]).

Теорема 2.11. Для любого полинома $P(z)=c_{0}+c_{1} z+\cdots+c_{n} z^{n}$ и любой точки $z=e^{i \varphi}$ на окружности $|z|=1$, в которой $\operatorname{Re} P(z)$ отлична от $L(P)$ и $H(P)$, справедливо неравенство

$$
\frac{\left|(\operatorname{Re} P(z))_{\varphi}^{\prime}\right|}{\sqrt{(\operatorname{Re} P(z)-L(P))(H(P)-\operatorname{Re} P(z))}} \leqslant n-1+\sqrt{\frac{2\left|c_{n}\right|}{H(P)-L(P)}} .
$$

Равенство в (2.16) достигается в случае $P(z)=c_{0}+c_{n} z^{n}$ для любых точек $z$, $|z|=1, z^{n} \neq \pm\left|c_{n}\right| / c_{n}$, где $c_{0}$ и $c_{n}$ - произвольные комплексные числа, $c_{n} \neq 0$.

Рассмотрим несколько следствий теоремы 2.11. Прежде всего заметим, что произвольный тригонометрический полином с вещественными коэффициентами

$$
T_{n}(\varphi)=a_{0}+\sum_{k=1}^{n}\left(a_{k} \cos k \varphi+b_{k} \sin k \varphi\right), \quad a_{n}^{2}+b_{n}^{2} \neq 0,
$$

можно рассматривать как действительную часть алгебраического полинома $P(z)$ на окружности $|z|=1$ :

$$
P(z)=a_{0}+\sum_{k=1}^{n}\left(a_{k}-i b_{k}\right) z^{k}
$$

Неравенство (2.16) в терминах таких полиномов примет вид

$$
\frac{\left|T_{n}^{\prime}(\varphi)\right|}{\sqrt{\left(T_{n}(\varphi)-L\left(T_{n}\right)\right)\left(H\left(T_{n}\right)-T_{n}(\varphi)\right)}} \leqslant n-1+\sqrt{\frac{2 \sqrt{a_{n}^{2}+b_{n}^{2}}}{H\left(T_{n}\right)-L\left(T_{n}\right)}},
$$

$-\infty<\varphi<\infty$, где $L\left(T_{n}\right)=\min _{[0,2 \pi]} T_{n}(\varphi), H\left(T_{n}\right)=\max _{[0,2 \pi]} T_{n}(\varphi)$. Подставляя в последнее неравенство вместо $T_{n}(\varphi)$ полином $T_{n l}(\varphi) \equiv T_{n}(l \varphi), l=1,2, \ldots$, деля обе части неравенства на $l$ и устремляя $l$ к бесконечности, получим в итоге

$$
\frac{\left|T_{n}^{\prime}(\varphi)\right|}{\sqrt{\left(T_{n}(\varphi)-L\left(T_{n}\right)\right)\left(H\left(T_{n}\right)-T_{n}(\varphi)\right)}} \leqslant n .
$$

Данное неравенство равносильно неравенству Бернштейна-Сегё [2; с. 232]:

$$
\left(T_{n}^{\prime}(\varphi)\right)^{2}+n^{2}\left(T_{n}(\varphi)\right)^{2} \leqslant n^{2}\left(\max _{[0,2 \pi]}\left|T_{n}(\varphi)\right|\right)^{2} .
$$

Отсюда приходим к первоначальному неравенству Бернштейна

$$
\left|T_{n}^{\prime}(\varphi)\right| \leqslant n \max _{[0,2 \pi]}\left|T_{n}(\varphi)\right|, \quad 0 \leqslant \varphi \leqslant 2 \pi,
$$

справедливому для любых тригонометрических полиномов $T_{n}(\varphi)[99 ;$ гл. I, п. 10]. Далее, замена среднего геометрического на среднее арифметическое в неравенстве (2.16) дает точную оценку для алгебраических полиномов:

$$
\left|\operatorname{Im}\left(z P^{\prime}(z)\right)\right| \leqslant(n-1) \frac{H(P)-L(P)}{2}+\sqrt{\left|c_{n}\right| \frac{H(P)-L(P)}{2}}
$$

с равенством для $P(z)=c_{0}+c_{n} z^{n}$ и некоторых $z$. Можно показать, что эта оценка не выполняется, если мнимую часть слева заменить на модуль. Учитывая соотношения

$$
H(P)-L(P) \leqslant|H(P)|+|L(P)| \leqslant 2 M(P)
$$


и пользуясь тем, что при вращении полинома $P(z) \rightarrow e^{i \theta} P(z)$ величина $M(P)$ не меняется, получим из последней оценки неравенство

$$
\left|P^{\prime}(z)\right| \leqslant(n-1) M(P)+\sqrt{\left|c_{n}\right| M(P)},
$$

справедливое для любого полинома $P(z)=c_{0}+c_{1} z+\cdots+c_{n} z^{n}$ и любых точек $z$ на окружности $|z|=1$. Равенство в $(2.17)$ достигается в случае $P(z)=c_{n} z^{n}$. Заметим, что в силу неравенства Коши $\left|c_{n}\right| \leqslant M(P)$, так что неравенство $(2.17)$ и предыдущие неравенства усиливают неравенство Бернштейна:

$$
M\left(P^{\prime}\right) \leqslant n M(P) .
$$

Функция из доказательства теоремы 2.4 приводит к теореме 2.12 (ср. с теоремой 1.8).

Tеорема 2.12. Для любого полинома $P(z)=c_{0}+c_{1} z+\cdots+c_{n} z^{n}$ и любой точки $z=e^{i \varphi}$ на окружности $|z|=1$, в которой модуль $|P(z)|$ отличен от $m(P)$ и $M(P)$, справедливо неравенство

$$
\begin{gathered}
\frac{\left|\left(|P(z)|^{2}\right)_{\varphi}^{\prime}\right|}{\sqrt{\left(|P(z)|^{2}-(m(P))^{2}\right)\left((M(P))^{2}-|P(z)|^{2}\right)}} \\
\leqslant n-1+2 \sqrt{\frac{\left|c_{0} c_{n}\right|}{(M(P))^{2}-(m(P))^{2}}} .
\end{gathered}
$$

Равенство в (2.18) достигается в случае $P(z)=c_{0}+c_{n} z^{n}$ для любих точек $z$, $|z|=1, z^{n} \neq \pm c_{0} / c_{n}$, где $c_{0}$ и $c_{n}$ - произвольные комплексные числа, удовлетворяющие условию $\left|c_{0}\right|=\left|c_{n}\right| \neq 0$.

Отметим точное неравенство

$$
\left|\left(|P(z)|^{2}\right)_{\varphi}^{\prime}\right| \leqslant n \frac{(M(P))^{2}-(m(P))^{2}}{2},
$$

которое получается из (2.18) заменой среднего геометрического на среднее арифметическое, а также заменой полинома $P(z)$ на $P\left(z^{l}\right)$ с последующим делением на $l$ и переходом к пределу при $l \rightarrow \infty$.

Теорема 2.13. Если полином $P(z)=c_{0}+c_{1} z+\cdots+c_{n} z^{n}$ не имеет нулей в круге $|z|<1$, то для любой точки $z=e^{i \varphi}$ на окружности $|z|=1$ выполняется неравенство

$$
(\arg P(z))_{\varphi}^{\prime} \leqslant \frac{n-1}{2}+\sqrt{\frac{\left|c_{n} / c_{0}\right|}{4}} .
$$

Равенство достигается для полинома $P(z)=c_{0}+c_{n} z^{n}$ и любъх точек $z$ на окружности $|z|=1$, где $c_{0}$ и $c_{n}$ - произвольные комплексные числа, удовлетворяющие условию $\left|c_{0}\right|=\left|c_{n}\right| \neq 0$.

ДокАЗАТЕЛЬСТво содержит функцию из леммы 2.5 .

Поскольку в теореме 2.13 нули полинома $P$ расположены на множестве $|z| \geqslant 1$, то $\left|c_{0} / c_{n}\right| \geqslant 1$ и неравенство (2.19) дает хорошо известное неравенство

$$
(\arg P(z))_{\varphi}^{\prime}=\operatorname{Re} \frac{z P^{\prime}(z)}{P(z)} \leqslant \frac{n}{2} .
$$


Отметим, что без ограничения на нули полинома $P$ величина $\operatorname{Re}\left(z P^{\prime}(z) / P(z)\right)$ может принимать на окружности $|z|=1$ сколь угодно большие значения. Однако для некоторых точек на этой окружности существует верхняя граница. Например, в точках $z$, где достигается максимум модуля $M(P)$, необходимо выполняется $\operatorname{Im}\left(z P^{\prime}(z) / P(z)\right)=0$ и, следовательно, $\left|\operatorname{Re}\left(z P^{\prime}(z) / P(z)\right)\right|=$ $\left|P^{\prime}(z)\right| / M(P)$. Из неравенства $(2.17)$ для таких точек вытекает, что

$$
\left|\operatorname{Re} \frac{z P^{\prime}(z)}{P(z)}\right| \leqslant n-1+\sqrt{\frac{\left|c_{n}\right|}{M(P)}} .
$$

Равенство достигается для полиномов $P(z)=c_{n} z^{n}$.

Функция из леммы 2.4 приводит к следующему результату.

Теорема 2.14. Если все нули полинома $P(z)=c_{0}+c_{1} z+\cdots+c_{n} z^{n}, c_{n} \neq 0$, лежат в круге $|z| \leqslant 1$, то для любой точки $z=e^{i \varphi}$ на окружности $|z|=1$ выполняется неравенство

$$
(\arg P(z))_{\varphi}^{\prime} \geqslant \frac{n+1}{2}-\sqrt{\frac{\left|c_{0} / c_{n}\right|}{4}} .
$$

Равенство достигается для полиномов из теоремы (2.13).

Левая часть неравенства (2.20) не превосходит $\left|z P^{\prime}(z) / P(z)\right|$, и, следовательно, в условиях теоремы 2.14 на окружности $|z|=1$ выполняется

$$
\left|P^{\prime}(z)\right| \geqslant|P(z)| \frac{n+1-\sqrt{\left|c_{0} / c_{n}\right|}}{2} .
$$

Равенство достигается для полинома $P(z)=c_{0}+c_{n} z^{n}$ в точках $z$, для которых $z^{n}=c_{0} / c_{n}$, где $\left|c_{0}\right|=\left|c_{n}\right| \neq 0$. Переходя в последнем неравенстве к максимуму, получим следующий результат:

$$
M\left(P^{\prime}\right) \geqslant M(P) \frac{n+1-\sqrt{\left|c_{0} / c_{n}\right|}}{2},
$$

что усиливает классическое неравенство П. Турана [100]:

$$
M\left(P^{\prime}\right) \geqslant \frac{n}{2} M(P),
$$

с равенством для полиномов $P(z)=c_{0}+c_{n} z^{n},\left|c_{0}\right|=\left|c_{n}\right| \neq 0$.

Предложенный метод доказательства позволяет выявить все случаи равенства в теоремах 2.7-2.14. Вместе с тем в известной нам литературе авторы ограничиваются указанием точности оценки и полинома, для которого выполняется равенство. Следуя "традиции" и не желая увеличивать объем статьи, затушевывая тем самым суть вопроса, отметим лишь, что равенство в теоремах 2.13 и 2.14 достигается для "self-inversive" полиномов, т. е. полиномов вида $P(z)=\alpha Q(z)$, где $Q(z)=z^{n} \overline{P(1 / \bar{z})}$ и $|\alpha|=1$.

Итак, лишь один только факт из теории однолистных функций (неравенство (1.12)) дает серию дифференциальных неравенств для полиномов. Приведем два примера применения других неравенств. Так, неравенство 3. Нехари [101; с. 258] и лемма 2.4 дают следующее утверждение [23; теорема 3]. 
Tеорема 2.15. Для полинома

$$
P(z)=c_{0}+c_{1} z+\cdots+c_{n} z^{n}, \quad c_{n} \neq 0, \quad c_{0} \neq 0,
$$

с вещественными коэффициентами $c_{k}, k=0,1, \ldots, n$, нули которого лежат в круге $|z| \leqslant 1$, д для точек $z$ окружности $|z|=1, z \neq \pm 1$, справедливо неравенство

$$
\begin{aligned}
\operatorname{Re} \frac{z P^{\prime}(z)}{P(z)} \geqslant \frac{n+1}{2} & \\
& -\frac{1}{2} \min \left\{\left|\frac{c_{0}}{c_{n}}\right|\left|\frac{\operatorname{Im} z}{\operatorname{Im}\left(z^{-n-1} P(z) / \overline{P(z)}\right)}\right|,\left|\frac{\operatorname{Im}\left(z^{-n-1} P(z) / \overline{P(z)}\right)}{\operatorname{Im} z}\right|\right\} .
\end{aligned}
$$

Равенство достигается для полиномов $P(z)$ с нулями на окружности $|z|=1$.

Неравенство (1.16) и функция

$$
f(z)=z^{-n} \frac{\overline{P(\bar{z})}}{P(1 / z)}, \quad|z|<1,
$$

приводят к теореме 2.16 (см. [8; теорема 4.3]).

Теорема 2.16. Предположим, что полином $P$ степени $n$ имеет в круге $|z|<1$ только один нуль первой кратности, и пусть точки $z_{k}, k=1, \ldots, m$, $m \geqslant 2$, расположены на окружности $|z|=1$ симметричным образом. Тогда справедливо неравенство

$$
\prod_{k=1}^{m} \max \left\{2 \operatorname{Re} \frac{z_{k} P^{\prime}\left(z_{k}\right)}{P\left(z_{k}\right)}-n, 0\right\} \leqslant 1 .
$$

Равенство достигается для полиномов $Р$ с одним нулем в начале и остальными на окружности $|z|=1$.

Предложенный подход позволяет получить теоремы, аналогичные приведенным выше, но уже для рациональных функций с предписанными полюсами (cм. [9]).

2.4. Неравенства для коэффициентов. Пусть $f$ - одна из функций, указанных в леммах $2.2-2.5$, построенная по заданному полиному $P, f(0)=0$. Обозначим через

$$
z=F(w)=\alpha w+\alpha_{1} w^{2}+\cdots, \quad|w|<1,
$$

обратную функцию для $w=f(z)$. Тогда функция

$$
\widetilde{F}(w)=\frac{F(w)}{\alpha}=w+\frac{\alpha_{1}}{\alpha} w^{2}+\cdots
$$

принадлежит известному классу $\mathrm{S}_{M}$ регулярных и однолистных в круге $|w|<1$ функций, удовлетворяющих условиям $\widetilde{F}(0)=0, \widetilde{F}^{\prime}(0)=1$ и $|\widetilde{F}(w)|<M$ при $|w|<1(M=1 /|\alpha|)$. K настоящему времени доказано немало теорем для функций данного класса (см., например, [84], [98], [102], [103]). В частности, известно немало оценок коэффициентов функции $\widetilde{F}(z)$. Из этих оценок вытекают некоторые оценки для коэффициентов исходного полинома $P$. Не рассматривая 
данный вопрос подробно, ограничимся, в основном, примерами оценок, вытекающих из известного неравенства

$$
\left|\frac{\alpha_{1}}{\alpha}\right| \leqslant 2(1-|\alpha|)
$$

[102; с. 94]. Если функция $f$ имеет в окрестности начала координат разложение

то указанное неравенство запишется в виде

$$
f(z)=\frac{1}{\alpha} z+\beta z^{2}+\cdots,
$$

$$
\left|\alpha^{2} \beta\right| \leqslant 2(1-|\alpha|) .
$$

Равенство достигается, например, в случае $f(z) \equiv z / \alpha,|\alpha|=1$.

ТеОрема 2.17. Для любого полинома $P(z)=c_{0}+c_{1} z+\cdots+c_{n-1} z^{n-1}+c_{n} z^{n}$ справедливо неравенство

$$
\left|c_{n-1}\right|+2\left|c_{n}\right| \leqslant H(P)-L(P) .
$$

Равенство достигается в случае $P(z)=c_{0}+c_{n} z^{n}$.

ДокАзАтельство. Достаточно проверить, что для функции $f$ из леммы 2.2

$$
\alpha=\frac{2 c_{n}}{H(P)-L(P)}, \quad \alpha^{2} \beta=-\frac{2 c_{n-1}}{H(P)-L(P)},
$$

и применить неравенство (2.21).

Заметим, что полученное неравенство содержит классическую оценку

$$
\left|c_{n}\right| \leqslant \frac{1}{2}(H(P)-L(P)),
$$

которая вытекает также из леммы Шварца: $|\alpha| \leqslant 1$.

ТеОРема 2.18. Если все нули полинома $P(z)=c_{0}+c_{1} z+\cdots+c_{n-1} z^{n-1}+c_{n} z^{n}$ лежат в круге $|z| \leqslant 1$, то справедливо неравенство

$$
\left|c_{n-1} \bar{c}_{0}-c_{n} \bar{c}_{1}\right| \leqslant 2\left|c_{n}\right|\left(\left|c_{n}\right|-\left|c_{0}\right|\right) .
$$

$B$ случае, когда все нули полинома $P(z)$ расположены на множестве $|z| \geqslant 1$, имеет место неравенство

$$
\left|c_{n-1} \bar{c}_{0}-c_{n} \bar{c}_{1}\right| \leqslant 2\left|c_{0}\right|\left(\left|c_{0}\right|-\left|c_{n}\right|\right) .
$$

Равенство в обоих случаях достигается для полиномов $P(z)$ с нулями на единичной окружности $|z|=1$.

ДоказАтельство. Рассмотрим первое неравенство. Можно считать, что все нули полинома $P(z)$ лежат в открытом круге $|z|<1$ и $c_{0} \neq 0, c_{n} \neq 0$. Введем функцию из леммы 2.4:

$$
f(z)=z^{n+1} \frac{P(1 / z)}{\overline{P(\bar{z})}}=\frac{1}{\alpha} z+\beta z^{2}+\cdots .
$$

Простые вычисления показывают, что на этот раз

$$
\alpha=\frac{\bar{c}_{0}}{c_{n}} \quad \text { и } \quad \beta=\frac{c_{n-1}}{\bar{c}_{0}}-\frac{c_{n} \bar{c}_{1}}{\left(\bar{c}_{0}\right)^{2}} .
$$

Осталось применить неравенство (2.21). Для доказательства второго неравенства достаточно применить первое неравенство к полиному $\widetilde{P}(z)=z^{n} P(1 / z)$, либо аналогично предыдущему воспользоваться леммой 2.5. Теорема доказана. 
Tеорема 2.19. Пусть $P(z)=c_{0}+c_{1} z+\cdots+c_{n-1} z^{n-1}+c_{n} z^{n}$. Тогда

$$
2\left|c_{n-1} \bar{c}_{0}+c_{n} \bar{c}_{1}\right| \leqslant M^{2}(P)-m^{2}(P)-4\left|c_{0} c_{n}\right|
$$

с равенством для полиномов $P(z)=c_{0}+c_{n} z^{n},\left|c_{0}\right|=\left|c_{n}\right|$.

Доказательство. Можно считать, что $c_{0} \neq 0$ и $c_{n} \neq 0$. Рассмотрим функцию

$$
w=f(z)=z^{1-n} \Phi\left(P\left(\frac{1}{z}\right) \overline{P(\bar{z})}\right)=\frac{1}{\alpha} z+\beta z^{2}+\cdots
$$

из доказательства теоремы 2.4. Для начальных коэффициентов этой функции выполняются равенства

$$
\alpha=\frac{4 c_{n} \bar{c}_{0}}{M^{2}(P)-m^{2}(P)}, \quad \alpha^{2} \beta=-\frac{4\left(c_{n-1} \bar{c}_{0}+c_{n} \bar{c}_{1}\right)}{M^{2}(P)-m^{2}(P)} .
$$

Осталось применить неравенство (2.21). В случае $P(z)=c_{0}+c_{n} z^{n},\left|c_{0}\right|=\left|c_{n}\right|$, имеем $|\alpha|=1$, и, следовательно, правая часть неравенства теоремы 2.19 равна нулю. Значит, левая часть также равна нулю, и мы имеем равенство. Теорема доказана.

Теорема 2.20. Для полинома $P(z)=c_{0}+c_{1} z+\cdots+c_{n-1} z^{n-1}+c_{n} z^{n}$ с вещественными коэфбициентами $c_{k}, k=0,1, \ldots, n$, и с критическими точками на отрезке $[-1,1]$ выполняется неравенство

$$
\left(\frac{\left|c_{n-1}\right|}{n}+\left|c_{n}\right|\right)^{n} \leqslant 2^{n-2}\left|c_{n}\right|^{n-1}(\bar{H}(P)-\bar{L}(P)) .
$$

Равенство достигается для полиномов $P(z)=a T_{n}(z)+b, a \in \mathbb{R} \backslash\{0\}, b \in \mathbb{R}$.

ДокАзАтЕльство осуществляется так же, как выше, но с применением функции из леммы 2.7. Случай равенства проверяется непосредственно.

Предположим, что в условиях теоремы 2.20 выполняется

$$
|P(z)| \leqslant 1, \quad \text { когда } z \in[-1,1] .
$$

Из неравенства (2.22) в этом случае следует, что

$$
\left(\frac{\left|c_{n-1}\right|}{n}+\left|c_{n}\right|\right)^{n} \leqslant\left(2\left|c_{n}\right|\right)^{n-1}
$$

Как впервые замечено А.В. Олесовым [34], неравенство (2.21), примененное к функции $f$ из леммы 2.3 , дает

$$
\left|c_{n-1}\right|+\left|c_{n}\right| \leqslant 2^{n-1} .
$$

Легко видеть, что неравенство (2.24) слабее (2.23), но оно справедливо без каких-либо ограничений на критические точки полинома $P$. Ранее В. А. Марковым было показано, что

$$
\frac{\left|c_{n-1}\right|}{n}+\left|c_{n}\right| \leqslant 2^{n-1}
$$

с равенством для полинома Чебышёва $T_{n}[104]$. 
Теорема 2.21. В условиях предъдущей теоремы справедливы неравенства

$$
\left|c_{n}\right| \geqslant\left(\frac{\left|c_{1}\right|}{n}\right)^{2 n} \frac{2^{3 n-2}}{(\bar{H}(P)-\bar{L}(P))^{2 n-1}}, \quad\left|c_{1}\right| \leqslant \frac{n}{2}(\bar{H}(P)-\bar{L}(P))
$$

с равенством для полинома Чебышёва $T_{n}$ при нечетном $n$.

ДокАзАТЕльство. Первое неравенство вытекает из теоремы 2.10, где необходимо положить $x=0$. Второе неравенство следует из первого, если воспользоваться классической оценкой

$$
\left|c_{n}\right| \leqslant 2^{n-2}(\bar{H}(P)-\bar{L}(P))
$$

Случай равенства проверяется непосредственно. Теорема доказана.

Пусть $P$ - полином степени $n$ с нулями на отрезке $[-1,1]$, удовлетворяющий условию $\bar{M}(P)=1$. Из следствия 2.4 и неравенства П. Турана [100] имеем другую оценку снизу модуля старшего коэффициента:

$$
\left|c_{n}\right| \geqslant\left(2\left(3 n^{3 / 2}\right)^{n}\right)^{-1}
$$

Эту оценку можно улучшить, применяя вместо неравенства П. Турана результат работы [105]. Интересно было бы знать точную нижнюю оценку $\left|c_{n}\right|$ в данных условиях, не зависящую от других коэффициентов полинома $P$.

Итак, мы рассмотрели некоторые применения лишь одного классического неравенства для коэффициентов однолистных функций. Построение однолистных функций с помощью леммы 2.1 и применение других оценок коэффициентов в различных классах однолистных функций ведет к многочисленным неравенствам для полиномов. В качестве примера приведем два утверждения, вытекающие из оценок Х. Сайджека и О. Тамми [106] (см. [11; §3]).

ТеОрема 2.22. Если полином $P(z)=c_{0}+c_{1} z+\cdots+c_{n-1} z^{n-1}+c_{n} z^{n}$ с вещественными коэфбициентами $c_{k}, k=0,1, \ldots, n, c_{n} \neq 0, n \geqslant 4$, удовлетворяет условию $|P(z)| \leqslant 1$ при $z \in[-1,1]$ и если $\lambda=\lambda(P):=\left|c_{n}\right| / 2^{n-1}$, то справедливы неравенства

$$
\begin{aligned}
\lambda & \leqslant 1, \\
\left|c_{n-1}\right| & \leqslant 2^{n-1}(1-\lambda), \\
\left|n c_{n}+4 c_{n-2}\right| & \leqslant 2^{n-1}\left(\lambda^{-1}-\lambda\right), \\
\left|(n-1) c_{n-1}+4 c_{n-3}\right| & \leqslant 2^{n-1}\left(\lambda^{-2}-\lambda^{-1}\right), \\
\left|n(n-1) c_{n}+8(n-2) c_{n-2}+32 c_{n-4}\right| & \leqslant 2^{n}\left(5 \lambda^{-3}-8 \lambda^{-2}+3 \lambda^{-1}\right) .
\end{aligned}
$$

Равенство во всех случаях достигается для полинома Чебышёва

$$
T_{n}(z)=2^{n-1} z^{n}+\cdots
$$

Первое неравенство в данной теореме классическое. Второе и третье получены А. В. Олесовым по существу тем же путем [34]. 
Теорема 2.23. Для полинома $P(z)=c_{0}+c_{1} z+\cdots+c_{n-1} z^{n-1}+c_{n} z^{n}, c_{n} \neq 0$, удовлетворяющего условию $|\operatorname{Re} P(z)| \leqslant 1$ при $|z|=1$, имеют место оценки

$$
\begin{aligned}
\left|c_{n}\right| & \leqslant 1, & & n \geqslant 1, \\
\left|c_{n-1}\right| & \leqslant 2\left(1-\left|c_{n}\right|\right), & & n \geqslant 2, \\
\left|c_{n-2} c_{n}\right| & \leqslant 1-\left|c_{n}\right|^{2}, & & n \geqslant 3, \\
\left|c_{n-k-1} c_{n}^{k}\right| & \leqslant \sum_{\nu=0}^{k-1} \gamma_{k \nu}\left|c_{n}\right|^{\nu}, & & k=2,3, \ldots, n-2, \quad n \geqslant 4, \\
\left|\operatorname{Re} c_{0}\right| & \leqslant 1-\left|c_{n}\right|, & n & =1, \\
\left|2 \operatorname{Re} c_{0}\right|\left|c_{n}\right| & \leqslant 1-\left|c_{n}\right|^{2}, & n & =2, \\
\left|2 \operatorname{Re} c_{0}\right|\left|c_{n}\right|^{n-1} & \leqslant \sum_{\nu=0}^{n-2} \gamma_{n-1 \nu}\left|c_{n}\right|^{\nu}, & & n \geqslant 3,
\end{aligned}
$$

əəe

$$
\gamma_{k \nu}=(-1)^{\nu} \frac{2}{\nu !} \frac{k-\nu}{k-\nu+1} \frac{(2 k-\nu-1) !}{[(k-\nu) !]^{2}}, \quad \nu=0, \ldots, k-1 .
$$

Равенство во всех оченках достигается в случае $P(z)=c_{n} z^{n},\left|c_{n}\right|=1$.

2.5. Полиномы с критическими значениями на отрезке. В данном разделе рассматриваются дополнения и уточнения некоторых результатов из разделов 2.2-2.4 в случае полиномов с фиксированным старшим коэффициентом и с критическими значениями на отрезке [12]. Для фиксированных $a$, $0<a<2^{n-1}$, и $n \geqslant 2$ обозначим через $\mathcal{P}_{n}^{a}$ совокупность всех полиномов $P(z)=a z^{n}+\cdots$ степени $n$ с вещественными коэффициентами, удовлетворяющих условию $\bar{M}(P) \leqslant 1$ и таких, что если в некоторой точке $z$ выполняется равенство $P^{\prime}(z)=0$, то необходимо $P(z) \in[-1,1]$. Ниже будут доказаны теоремы покрытия, неравенство бернштейновского типа и неравенства для коэффициентов полиномов класса $\mathcal{P}_{n}^{a}$. Экстремальным в каждом случае будет полином

$$
T_{n}^{a}(z):=T_{n}(b z+b-1)=a z^{n}+\cdots,
$$

где $b=\left(a \cdot 2^{1-n}\right)^{1 / n}<1$. Пусть

$$
\eta=\Phi(\omega)=\omega+\sqrt{\omega^{2}-1}
$$

означает ту ветвь аналитической функции, обратной функции Жуковского, которая конформно и однолистно отображает внешность отрезка $[-1,1]$ на круг $|\eta|<1$. Ключевую роль в приведенных ниже доказательствах играет функция

$$
w=g(\zeta):=\left[\Phi\left(P\left(\frac{\zeta+1 / \zeta}{2}\right)\right)\right]^{1 / n}=\frac{1}{b} \zeta+\cdots,
$$

которая согласно лемме 2.7 конформно и однолистно отображает область $\{\zeta:|\zeta|<1, P((\zeta+1 / \zeta) / 2) \notin[-1,1]\}$ на круг $|w|<1$. Здесь $P \in \mathcal{P}_{n}^{a}$ и $1^{1 / n}=1$. Соответственно, обратная $w=g(\zeta)$ функция

$$
\zeta=h(w)=\left(a \cdot 2^{1-n}\right)^{1 / n} w+\cdots
$$


принадлежит классу ограниченных однолистных в круге $|w|<1$ функций, экстремальные свойства которых хорошо изучены в литературе (см., например, [84]).

Обозначим через $\zeta=p(w ; b)$ функцию Пика, заданную соотношениями

$$
\frac{\zeta}{(1+\zeta)^{2}}=\frac{b w}{(1+w)^{2}}, \quad|w|<1, \quad|\zeta|<1, \quad \zeta \notin[d, 1],
$$

где $d=2 b^{-1}-1-2 \sqrt{b^{-1}\left(b^{-1}-1\right)}, 0<b<1$.

Теорема 2.24. Для любого полинома $P$ класса $\mathcal{P}_{n}^{a}$ и любого числа $\rho$, $0<\rho<1$, прообраз эллипса с фбокусами в точках \pm 1 и большой полуосъю $(\rho+1 / \rho) / 2$ лежит в кольцевой области $G(n, a, \rho)$, ограниченной двумя эллипсами с бокусами в точках \pm 1 и большими полуосями, равными

$$
\frac{1}{2}\left(-p\left(-\rho^{1 / n} ; b\right)-\frac{1}{p\left(-\rho^{1 / n} ; b\right)}\right) \quad u \quad \frac{1}{2}\left(p\left(\rho^{1 / n} ; b\right)+\frac{1}{p\left(\rho^{1 / n} ; b\right)}\right),
$$

где $b=\left(a \cdot 2^{1-n}\right)^{1 / n}$. Исключение составляет случай, когда $P(z) \equiv T_{n}^{a}(z)$ и, следовательно, прообраз указанного эллипса есть эллипс, все точки которого принадлежат области $G(n, a, \rho)$, кроме точек

$$
\frac{1}{2}\left(p\left(-\rho^{1 / n} ; b\right)+\frac{1}{p\left(-\rho^{1 / n} ; b\right)}\right) \quad u \quad \frac{1}{2}\left(p\left(\rho^{1 / n} ; b\right)+\frac{1}{p\left(\rho^{1 / n} ; b\right)}\right),
$$

которые лежат на ее границе.

ДокАЗАТЕЛЬство. По заданному полиному $P$ класса $\mathcal{P}_{n}^{a}$ построим функцию $w=g(\zeta)$ из $(2.25)$ и рассмотрим обратное отображение $\zeta=h(w)=b w+\cdot \cdots$. Для любой точки $w$ на окружности $|w|=\rho^{1 / n}$ справедливы неравенства Пика $[84 ;$ с. 55$]$

$$
p\left(\rho^{1 / n} ; b\right) \leqslant|h(w)| \leqslant-p\left(-\rho^{1 / n} ; b\right) .
$$

Это означает, что точка $z=(\zeta+1 / \zeta) / 2, \zeta=h(w)$, принадлежит замыканию области $G(n, a, \rho)$, в то время как точка $P(z)=\left(w^{n}+1 / w^{n}\right) / 2$ является произвольной точкой эллипса с фокусами в точках \pm 1 и большой полуосью $(\rho+1 / \rho) / 2$. Принадлежность точки $z=(\zeta+1 / \zeta) / 2$ границе области $G(n, a, \rho)$ означает равенство в левом либо правом неравенстве в $(2.26)$ для некоторой точки $w$. Хорошо известно, что в этом случае $h(w) \equiv \alpha p(\beta w ; b)$, где $\alpha$ и $\beta-$ комплексные числа, удовлетворяющие условию $|\alpha|=|\beta|=1$. Тогда из представления (2.25) вытекает, что $\alpha=\beta=1$ и полином $P$ отображает отрезок $[-1,(d+1 / d) / 2]$ на отрезок $[-1,1]$, где $d-$ из определения функции Пика. Следовательно, полином $P((z+1-b) / b)=2^{n-1} z^{n}+\cdots$ отображает отрезок $[-1,1]$ на себя (т. е. $\left.(d+1 / d) / 2=2 b^{-1}-1\right)$. Отсюда вытекает, что $P((z+1-b) / b) \equiv T_{n}(z)$ и $P(z) \equiv T_{n}^{a}(z)$. Характер отображения, осуществляемого полиномом $T_{n}^{a}(z)$, непосредственно виден из его определения. Теорема доказана.

Теорема 2.25. Если полином $P$ принадлежит классу $\mathcal{P}_{n}^{a}$, то для любого числа $t, 0<t<1$, образ эллипса с фокусами в точках \pm 1 и большой полуосъю $(t+1 / t) / 2$ при отображении этим полиномом лежит в конечной области, ограниченной эллипсом с фокусами в точках \pm 1 и большой полуосъю $(\rho+1 / \rho) / 2$, где $p\left(-\rho^{1 / n} ; b\right)=-t, b=\left(a \cdot 2^{1-n}\right)^{1 / n}$. Исключение составляет случай, когда $P(z) \equiv T_{n}^{a}(z)$ и, следовательно, все точки образа эллипса 
принадлежат указанной выше области, кроме точки - $(\rho+1 / \rho) / 2$, которая лежит на ее границе.

ДокаЗАТЕЛЬСтво. Легко видеть, что для любого $t, 0<t<1$, уравнение

$$
p\left(-\rho^{1 / n} ; b\right)=-t
$$

имеет единственное решение $\rho, 0<\rho<1$. Из правого неравенства в $(2.26)$ заключаем, что если точка $z=(\zeta+1 / \zeta) / 2, \zeta=h(w)$, принадлежит эллипсу с фокусами в точках \pm 1 и большой полуосью $(t+1 / t) / 2$, т. е. если $|\zeta|=t$, то соответствующая точка $w=g(\zeta)$ удовлетворяет неравенству $|w| \geqslant \rho^{1 / n}$. Это означает принадлежность точки $P(z)=\left(w^{n}+1 / w^{n}\right) / 2$ замыканию области, указанной в формулировке теоремы 2.25 , причем если $P(z)$ лежит на границе этой области, то $|w|=\rho^{1 / n}$ и в правом неравенстве $(2.26)$ имеет место знак равенства. В случае равенства в (2.26), как и при доказательстве теоремы 2.24 , приходим к тождеству $P(z) \equiv T_{n}^{a}(z)$. Теорема доказана.

Заметим, что при отображении полиномом $T_{n}^{a}, 0<a<2^{n-1}$, образ эллипса с фокусами в точках \pm 1 не является эллипсом (в отличие от случая полинома Чебышёва $T_{n}$, т. е. когда $a=2^{n-1}$ ). Повторяя доказательство теоремы 2.25 , но с привлечением левого неравенства в (2.26), можно получить принадлежность образа эллипса с фокусами в точках \pm 1 и большой полуосью $(t+1 / t) / 2$ "внешности" некоторого эллипса, но только в случае $t<d$.

Теорема 2.26. Если полином $P$ принадлежит классу $\mathcal{P}_{n}^{a}$, то для любого $z \in[-1,1]$ выполняется неравенство

$$
\left|P^{\prime}(z) \sin \left(\frac{1}{n} \arccos P(z)\right)\right| \leqslant n\left(a \cdot 2^{1-n}\right)^{1 / n} \sqrt{1-P^{2}(z)} .
$$

Равенство в (2.27) для любого $z \in[-1,1]$ достигается в случае $P(z) \equiv T_{n}^{a}(z)$.

ДокАЗАТЕЛЬство. По заданному полиному $P \in \mathcal{P}_{n}^{a}$ построим функцию $w=g(\zeta)$ из $(2.25)$ и рассмотрим обратную к ней функцию $\zeta=h(w)=(a \times$ $\left.2^{1-n}\right)^{1 / n} w+\cdots$. Пусть

$$
\zeta_{1}=e^{i \theta}, \quad \zeta_{2}=e^{-i \theta}, \quad w_{k}=g\left(\zeta_{k}\right), \quad k=1,2, \quad 0<\theta<\pi,
$$

и пусть в точках $w_{k}, k=1,2$, существуют производные $h^{\prime}\left(w_{k}\right), k=1,2$, а в точках $\zeta_{k}, k=1,2,-$ производные $g^{\prime}\left(\zeta_{k}\right), k=1,2$. Применяя к функции $\zeta=h(w)$ результат Х. Поммеренке и А. Васильева [107; неравенство (1.1)] и выписывая его в терминах функции $w=g(\zeta)$, получаем

$$
\left|\frac{\zeta_{1}-\zeta_{2}}{g\left(\zeta_{1}\right)-g\left(\zeta_{2}\right)}\right| \geqslant\left(a \cdot 2^{1-n}\right)^{-1 / n}\left|g^{\prime}\left(\zeta_{1}\right) g^{\prime}\left(\zeta_{2}\right)\right|^{1 / 2} .
$$

Положим $z=\left(\zeta_{1}+1 / \zeta_{1}\right) / 2=\left(\zeta_{2}+1 / \zeta_{2}\right) / 2$. Простые вычисления дают

$$
\begin{gathered}
\left|\zeta_{1}-\zeta_{2}\right|=2 \sqrt{1-z^{2}}, \quad\left|g\left(\zeta_{1}\right)-g\left(\zeta_{2}\right)\right|=2\left|\sin \left(\frac{1}{n} \arccos P(z)\right)\right| \\
\left|g^{\prime}\left(\zeta_{k}\right)\right|=\frac{d|g|}{d|\zeta|}=\frac{1}{n}|\Phi(P(z))|^{1 / n-1} \frac{\left|P^{\prime}(z)\right|\left|\left(1-\zeta_{k}^{-2}\right) / 2\right|}{\sqrt{1-P^{2}(z)}}=\frac{1}{n} \frac{\left|P^{\prime}(z)\right| \sqrt{1-z^{2}}}{\sqrt{1-P^{2}(z)}} \\
k=1,2 .
\end{gathered}
$$


Подставляя выписанные соотношения в (2.28), приходим к неравенству (2.27) для всех точек $z$ отрезка $[-1,1]$ за исключением, быть может, конечного числа таких точек. Предельным переходом убеждаемся в справедливости неравенства (2.27) на всем отрезке $[-1,1]$. Случай равенства проверяется непосредственно. Теорема доказана.

Неравенство (2.27) содержит неравенство следствия 2.4. Действительно, обозначим $\cos ((1 / n) \arccos P(z))$ через $\xi$, так что $P(z)=T_{n}(\xi)$. Из неравенства (2.27) с привлечением леммы 4.1 работы [8] имеем последовательно

$$
\begin{aligned}
\left|P^{\prime}(z)\right| & \leqslant n\left(a \cdot 2^{1-n}\right)^{1 / n} \frac{\sqrt{1-\left(T_{n}(\xi)\right)^{2}}}{\sqrt{1-\xi^{2}}} \\
& \leqslant n\left(a \cdot 2^{1-n}\right)^{1 / n} \max \left\{\sqrt{\left|T_{n}^{\prime}(\xi)\right|}: \xi \in[-1,1]\right\}=n^{2}\left(a \cdot 2^{1-n}\right)^{1 / n} .
\end{aligned}
$$

Пусть, как и выше, $\zeta=h(w)$ - функция, обратная к функции $(2.25)$, где $P \in \mathcal{P}_{n}^{a}$. Применяя к функции $\zeta=h(w)$ известные оценки коэффициентов для регулярных и однолистных функций, приходим к некоторым неравенствам для коэффициентов полинома $P(z)$. Ограничимся следующим результатом. ТЕорема 2.27. Пусть полином $P(z)=a z^{n}+\sum_{k=0}^{n-1} c_{k} z^{k}$ принадлежит клас-
су $\mathcal{P}_{n}^{a}$. Тогда справедливы неравенства

$$
\begin{gathered}
n a+\left|c_{n-1}\right| \leqslant n(2 a)^{(n-1) / n}, \\
n^{2} a^{2}+\left|n^{2} a^{2}+4 n a c_{n-2}-2(n-1) c_{n-1}^{2}\right| \leqslant n^{2}(2 a)^{2(n-1) / n} .
\end{gathered}
$$

Равенство в обоих случаях достигается для полинома $T_{n}^{a}$.

ДокАЗАТЕЛЬСТво. Функция

$$
w=\frac{\left(a \cdot 2^{1-n}\right)^{1 / n}}{h(1 / z)}=z+\sum_{k=0}^{\infty} A_{k} z^{-k}, \quad|z|>1,
$$

принадлежит классу $\Sigma_{b}, b=\left(a \cdot 2^{1-n}\right)^{1 / n}$, работы Х. Сайджека и О. Тамми [106]. В [106] получены точные оценки всех коэффициентов в разложении обратной функции

$$
z=I(w)=w-\sum_{k=0}^{\infty} \beta_{k} w^{-k}, \quad|w|>1
$$

В частности, имеют место неравенства [106; с. 92]

$$
\left|\beta_{0}\right| \leqslant 2(1-b), \quad\left|\beta_{1}\right| \leqslant 1-b^{2} .
$$

Выписанные неравенства относятся к классическим, и они были доказаны также ранее другими авторами различными методами. Элементарные вычисления дают

$$
I(w)=\frac{1}{g(b / w)}=w+\frac{2 c_{n-1} b}{n a}+\left[1+\frac{4 c_{n-2}}{n a}-\frac{2(n-1) c_{n-1}^{2}}{n^{2} a^{2}}\right] \frac{b^{2}}{w}+\cdots,
$$

где функция $w=g(\zeta)$ задана соотношением (2.25). Поэтому из неравенств $(2.29)$ вытекают неравенства теоремы 2.27. В случае $P(z)=T_{n}^{a}(z)$ функция $\zeta=h(w)$ является функцией Пика, для которой в неравенствах (2.29), как известно, достигается знак равенства. Этим завершается доказательство теоремы 2.27. 
Учитывая полученные здесь результаты, можно предположить, что полином $T_{n}^{a}$ является экстремальным также в аналогичных задачах для более широкого класса полиномов (без ограничения на критические значения).

2.6. О полиномах с криволинейной мажорантой. Следуя методике, разработанной в разделах 2.1-2.4, рассмотрим здесь точные оценки коэффициентов, теоремы покрытия и неравенства для производных полиномов вида

$$
P_{n}(z)=c_{0}+\cdots+c_{n} z^{n}, \quad c_{n} \neq 0, \quad c_{k} \in \mathbb{R}, \quad k=0,1, \ldots, n, \quad n \geqslant 1,
$$

удовлетворяющих одному из следующих условий:

$$
\begin{aligned}
& \left|P(z) \sqrt{1-z^{2}}\right| \leqslant 1, \quad z \in[-1,1], \\
& \left|P(z) \sqrt{\frac{1+z}{2}}\right| \leqslant 1, \quad z \in[-1,1], \\
& \left|P(z) \sqrt{\frac{1-z}{2}}\right| \leqslant 1, \quad z \in[-1,1] .
\end{aligned}
$$

Обозначим через $\mathcal{P} \mathcal{U}_{n}$ класс полиномов вида (2.30), удовлетворяющих условию $(2.31)$, через $\mathcal{P} \mathcal{V}_{n}-$ класс полиномов (2.30), удовлетворяющих условию (2.32), и, наконец, пусть $\mathcal{P} \mathcal{W}_{n}$ означает класс полиномов $(2.30)$, удовлетворяющих условию (2.33). В приведенных ниже результатах полиномы Чебышёва второго, третьего и четвертого рода $U_{n}(z), V_{n}(z)$ и $W_{n}(z)$ являются экстремальными в классах соответственно $\mathcal{P} \mathcal{U}_{n}, \mathcal{P} \mathcal{V}_{n}$ и $\mathcal{P} \mathcal{W}_{n}$. Хорошо известны представления этих полиномов:

$$
\begin{aligned}
U_{n}(z) & =\frac{\left(z+\sqrt{z^{2}-1}\right)^{n+1}-\left(z-\sqrt{z^{2}-1}\right)^{n+1}}{2 \sqrt{z^{2}-1}}, \\
V_{n}(z) & =\frac{\left(z+\sqrt{z^{2}-1}\right)^{n+1 / 2}+\left(z-\sqrt{z^{2}-1}\right)^{n+1 / 2}}{\left(z+\sqrt{z^{2}-1}\right)^{1 / 2}+\left(z-\sqrt{z^{2}-1}\right)^{1 / 2}}, \\
W_{n}(z) & =\frac{\left(z+\sqrt{z^{2}-1}\right)^{n+1 / 2}-\left(z-\sqrt{z^{2}-1}\right)^{n+1 / 2}}{\left(z+\sqrt{z^{2}-1}\right)^{1 / 2}-\left(z-\sqrt{z^{2}-1}\right)^{1 / 2}}
\end{aligned}
$$

(см., например, [108], [109]).

Обозначим через

$$
\zeta=\Phi(\omega)=\omega-\sqrt{\omega^{2}-1}
$$

функцию, конформно и однолистно отображающую внешность отрезка $[-1,1]$ на круг $|\zeta|<1$ так, что $\Phi(\infty)=0, \Phi(-1)=-1$. Справедливость следующих двух утверждений проверяется непосредственно.

Лемма 2.8. Если полином $P$ принадлежит классу $\mathcal{P \mathcal { U } _ { n }}$, то функиия

$$
w=f_{1}(z):=z^{-n} \Phi\left[\frac{i}{2}\left(z-\frac{1}{z}\right) P\left(\frac{1}{2}\left(z+\frac{1}{z}\right)\right)\right],
$$

заданная на множестве

$$
B_{1}:=\left\{z:|z|<1, \frac{i}{2}\left(z-\frac{1}{z}\right) P\left(\frac{1}{2}\left(z+\frac{1}{z}\right)\right) \notin[-1,1],\left|f_{1}(z)\right| \neq 1\right\},
$$

удовлетворяет условиям леммы 2.1 . 
Лемма 2.9. Если полином $P$ принадлежит классу $\mathcal{P} \mathcal{V}_{n}$, то функиия

$$
w=f_{2}(z):=z^{-2 n} \Phi\left[\frac{1}{2}\left(z+\frac{1}{z}\right) P\left(\frac{1}{2}\left(z^{2}+\frac{1}{z^{2}}\right)\right)\right],
$$

заданная на множестве

$$
B_{2}:=\left\{z:|z|<1, \frac{1}{2}\left(z+\frac{1}{z}\right) P\left(\frac{1}{2}\left(z^{2}+\frac{1}{z^{2}}\right)\right) \notin[-1,1],\left|f_{2}(z)\right| \neq 1\right\},
$$

удовлетворяет условиям леммы 2.1 .

Из представлений полиномов Чебышёва, указанных выше, легко заключаем, что если в лемме 2.8 в качестве полинома $P$ взять $U_{n}$, то $f_{1}(z) \equiv i z$, а если в лемме 2.9 положить $P=V_{n}$, то $f_{2}(z) \equiv z$.

Теорема 2.28. Для полиномов $P(z)=\sum_{k=0}^{n} c_{k} z^{k}$ класса $\mathcal{P U}_{n}$ имеют место точные оценки

$$
\left|c_{n-1}\right|+\left|c_{n}\right| \leqslant 2^{n}, \quad\left|c_{n}\right|^{2}+\left|(n-1) c_{n}^{2}+4 c_{n} c_{n-2}\right| \leqslant 4^{n} .
$$

Равенство достигается в случае полинома Чебъшёва $U_{n}$.

ДокАзАТЕЛЬство. Пусть функция $f_{1}$ из леммы 2.8 имеет в некоторой окрестности начала координат разложение

$$
f_{1}(z)=\beta_{1} z+\beta_{2} z^{2}+\beta_{3} z^{3}+\cdots .
$$

Ввиду леммы 2.1 существует функция

$$
z=F(w)=\alpha_{1} w+\alpha_{2} w^{2}+\alpha_{3} w^{3}+\cdots
$$

такая, что $f_{1}(F(w)) \equiv w$ в круге $U_{w}$. Коэффициенты функций $F$ и $f_{1}$ связаны соотношениями

$$
\alpha_{1}=\frac{1}{\beta_{1}}, \quad \alpha_{2}=-\frac{\beta_{2}}{\beta_{1}^{3}}, \quad \alpha_{3}=\frac{2 \beta_{2}^{2}-\beta_{3} \beta_{1}}{\beta_{1}^{5}} .
$$

С другой стороны, из определения функции $f_{1}$ имеем

$$
\begin{aligned}
& \beta_{1} z^{n+1}+\beta_{2} z^{n+2}+\beta_{3} z^{n+3}+\cdots=\Phi\left[\frac{i}{2}\left(z-\frac{1}{z}\right) P\left(\frac{1}{2}\left(z+\frac{1}{z}\right)\right)\right] \\
& =\Phi\left[\frac{i}{2}\left(-\frac{c_{n}}{2^{n}} \frac{1}{z^{n+1}}-\frac{c_{n-1}}{2^{n-1}} \frac{1}{z^{n}}+\left((1-n) \frac{c_{n}}{2^{n}}-\frac{c_{n-2}}{2^{n-2}}\right) \frac{1}{z^{n-1}}+\cdots\right)\right]
\end{aligned}
$$

в некоторой окрестности начала координат. Отсюда следует, что

$$
\beta_{1}=i \frac{2^{n}}{c_{n}}, \quad \frac{\beta_{2}}{\beta_{1}^{2}}=i \frac{c_{n-1}}{2^{n-1}}, \quad \frac{\beta_{2}^{2}-\beta_{3} \beta_{1}}{\beta_{1}^{3}}=i\left((1-n) \frac{c_{n}}{2^{n}}-\frac{c_{n-2}}{2^{n-2}}\right) .
$$

Учитывая соотношения (2.34) и классические оценки

$$
\left|\frac{\alpha_{2}}{\alpha_{1}}\right| \leqslant 2\left(1-\left|\alpha_{1}\right|\right), \quad\left|\left(\frac{\alpha_{2}}{\alpha_{1}}\right)^{2}-\frac{\alpha_{3}}{\alpha_{1}}\right| \leqslant 1-\left|\alpha_{1}\right|^{2}
$$

(см., например, [102; с. 94]), получаем требуемый результат. Экстремальность полинома $U_{n}(z)$ проверяется непосредственно. Теорема доказана. 
Первое неравенство теоремы 2.28 содержит оценку

$$
\left|c_{n}\right| \leqslant 2^{n}
$$

эквивалентную свойству полинома $U_{n}(z) \cdot 2^{-n}$ иметь наименьшее отклонение от нуля с весом $\sqrt{1-z^{2}}$ на отрезке $[-1,1]$ среди полиномов вида $P(z)=z^{n}+\cdots$ (см. [109]). Таким образом, наши оценки можно рассматривать как уточнения этого классического свойства полинома с участием старших коэффициентов. Аналогично, полином Чебышёва третьего рода является экстремальным в следующей проблеме.

Теорема 2.29. Для полиномов $P(z)=\sum_{k=0}^{n} c_{k} z^{k}$ класса $\mathcal{P} \mathcal{V}_{n}$ имеют место точные оченки

$$
\left|c_{n}\right| \leqslant 2^{n}, \quad\left|c_{n}\right|^{2}+\left|c_{n}^{2}+2 c_{n} c_{n-1}\right| \leqslant 4^{n} .
$$

Равенство достигается в случае полинома Чебышёва $V_{n}$.

ДокАЗАТЕЛЬство дословно повторяет доказательство теоремы 2.28 с заменой функции $f_{1}$ из леммы 2.8 на функцию $f_{2}$ из леммы 2.9 .

Следующие два утверждения вытекают из неравенств Пика, примененных к обратным функциям из лемм 2.8 и 2.9 , подобно доказательству теоремы 2.1 .

Теорема 2.30. Пусть полином $P(z)=c_{0}+\cdots+c_{n} z^{n}$ принадлежит классу $\mathcal{P U}_{n}$, и пусть $r>1$. Тогда образ эллипса $|z-1|+|z+1|=r+1 / r$ при отображении $\omega=\sqrt{1-z^{2}} P(z)$ лежит внутри эллипса с фокусами в точках -1 и 1 и большой осъю, равной $x_{0} r^{-n}+r^{n} / x_{0} \leqslant r^{-n-1}+r^{n+1}$, где $x_{0}-$ корень уравнения

$$
\left|c_{n}\right|(1-r)^{2} x=r \cdot 2^{n}(1-x)^{2},
$$

принадлежащий промежутку $1 / r \leqslant x<1$. Для полинома $U_{n}(z)$ имеем $x_{0}=$ $1 / r$, и образом указанного выше эллипса при отображении $\omega=\sqrt{1-z^{2}} U_{n}(z)$ является эллипс с фокусами в точках -1 и 1 и большой осъю величины $r^{-n-1}+$ $r^{n+1}$.

ТеОрема 2.31. Пусть полином $P(z)=c_{0}+\cdots+c_{n} z^{n}$ принадлежит класcу $\mathcal{P} \mathcal{V}_{n}$, u пусть $r>1$. Тогда образ эллипса $|z-1|+|z+1|=r^{2}+1 / r^{2}$ при отображении $\omega=\sqrt{(1+z) / 2} P(z)$ лежит внутри эллипса с фокусами в точках -1 и 1 и большой осъю, равной $x_{0} r^{-2 n}+r^{2 n} / x_{0} \leqslant r^{-2 n-1}+r^{2 n+1}$, где $x_{0}$ - корень уравнения (2.35) на промежутке $1 / r \leqslant x_{0}<1$. Для полинома $V_{n}(z)$ имеем $x_{0}=1 / r$, и образом указанного выше эллипса при отображении $\omega=\sqrt{(1+z) / 2} V_{n}(z)$ является эллипс с фокусами в точках -1 и 1 и большой осъю величины $r^{-2 n-1}+r^{2 n+1}$.

Доказательство, аналогичное доказательству теоремы 2.7 и использующее неравенства (1.12) и лемму 2.8, дает следующий результат.

Теорема 2.32. Если полином $P(z)=c_{0}+\cdots+c_{n} z^{n}, c_{n} \neq 0$, принадлежит классу $\mathcal{P U}_{n}$, то для любого $x \in[-1,1]$ справедливо неравенство

$$
\left|x P(x)-\left(1-x^{2}\right) P^{\prime}(x)\right| \leqslant\left(n+\sqrt{2^{-n}\left|c_{n}\right|}\right) \sqrt{1-\left(1-x^{2}\right) P^{2}(x)} .
$$

Равенство в (2.36) для любого $x \in[-1,1]$ достигается в случае, когда $P$ совпадает с полиномом Чебышёва $U_{n}$. 
Для сравнения с неравенством (2.36) приведем результат М. Лачанса [110]:

$$
\left(1-x^{2}\right)\left|P^{\prime}(x)\right| \leqslant 2(n+1), \quad x \in[-1,1],
$$

и неравенство, вытекающее из теоремы К. И. Рахмана [111]:

$$
\left|2 x P(x)-\left(1-x^{2}\right) P^{\prime}(x)\right| \leqslant 2(n+1), \quad x \in[-1,1] .
$$

Оба неравенства справедливы для полиномов класса $\mathcal{P} \mathcal{U}_{n}$. Легко показать, что из теоремы 2.32 и классического неравенства Шура

$$
\max \{|P(x)|: x \in[-1,1]\} \leqslant(n+1) \max \left\{\left|P(x) \sqrt{1-x^{2}}\right|: x \in[-1,1]\right\}
$$

следуют усиления этих неравенств. Более того, из теоремы 2.32 вытекает следующее уточнение неравенства (2.37) (см. [23]).

Теорема 2.33. Предположим, что полином $P(z)=c_{0}+\cdots+c_{n} z^{n}, c_{n} \neq 0$, принадлежит классу $\mathcal{P U}_{n}$. Тогда для любого $x \in[-1,1]$ справедливо неравенство

$$
|P(x)| \leqslant n+\sqrt{\frac{\left|c_{n} x^{n}\right|}{2^{n}}} \leqslant n+1 .
$$

Равенство достигается для полинома Чебышёва $U_{n}(z)$ в точке $x=1$.

ДоказАтельство. Для заданного полинома $P$, удовлетворяющего условию теоремы, и фиксированного $x \in[-1,1]$ рассмотрим полином

$$
Q(y):=P(x y)=c_{0}+\cdots+c_{n} x^{n} y^{n} .
$$

Поскольку для любых $y \in[-1,1]$ выполняется

$$
|Q(y)| \sqrt{1-y^{2}} \leqslant|P(x y)| \sqrt{1-(x y)^{2}} \leqslant 1,
$$

то к полиному $Q(y)$ применима теорема 2.32. Согласно этой теореме и неравенству $\left|c_{n}\right| \leqslant 2^{n}$, имеем

$$
|P(x)|=|Q(1)| \leqslant n+\sqrt{\frac{\left|c_{n} x^{n}\right|}{2^{n}}} \leqslant n+1 .
$$

Случай равенства проверяется непосредственно. Теорема доказана.

Неравенство Шура (2.37) эквивалентно "итоговому" неравенству в теореме 2.33.

Стандартным путем из неравенства бернштейновского типа (2.14) и уточнения неравенства Шура приходим к следующему усилению классического неравенства Маркова.

Теорема 2.34. Если полином $P(z)=c_{0}+\cdots+c_{n} z^{n}, c_{n} \neq 0$, с вещественнъми коэфбициентами удовлетворяет условию $\bar{M}(P) \leqslant 1$, то для любого $x \in[-1,1]$ справедливо неравенство

$$
\left|P^{\prime}(x)\right| \leqslant(n-1)\left(n-1+\sqrt{\frac{\left|c_{n}\right|}{2^{n-1}}}\right)+\sqrt{\frac{n\left|c_{n} x^{n-1}\right|}{2^{n-1}}} \sqrt{n-1+\sqrt{\frac{\left|c_{n}\right|}{2^{n-1}}}} .
$$

Равенство достигается для полинома Чебишёва $T_{n}(z)$ в точке $x=1$. 
Оценивая правую часть неравенства теоремы 2.34 сверху, получим

$$
\left|P^{\prime}(x)\right| \leqslant\left(n-1+\sqrt{\frac{\left|c_{n} x^{n-1}\right|}{2^{n-1}}}\right) n \leqslant n^{2}, \quad x \in[-1,1],
$$

где "итоговое" неравенство есть неравенство Маркова.

Следующий результат получается из теоремы 2.31 предельным переходом при $r \rightarrow 1$ (см. [24]).

ТеОрема 2.35. Если полином $P(z)=c_{0}+\cdots+c_{n} z^{n}$ принадлежит классу $\mathcal{P} \mathcal{V}_{n}$, то для любого $x \in[-1,1]$ справедливо неравенство

$$
\sqrt{1-x}\left|P(x)+2(x+1) P^{\prime}(x)\right| \leqslant\left(\sqrt{\frac{\left|c_{n}\right|}{2^{n}}}+2 n\right) \sqrt{2-(x+1) P^{2}(x)} .
$$

Равенство в каждой точке $x \in[-1,1]$ достигается для полинома Чебышёва $V_{n}$.

Приведем теперь утверждение, содержащее аналог неравенства Шура (2.37).

Теорема 2.36. Пусть полином $P(z)=c_{0}+\cdots+c_{n} z^{n}$ принадлежит классу $\mathcal{P} \mathcal{V}_{n}$. Тогда для любого $x \in[-1,1]$ справедливо неравенство

$$
|P(x)| \leqslant 2 n+\sqrt{\frac{\left|c_{n}(1-x)^{n}\right|}{4^{n}}} \leqslant 2 n+1 .
$$

Равенство достигается для полинома Чебышёва $V_{n}$ в точке $x=-1$.

ДоказАтельство. Для заданного полинома $P$ класса $\mathcal{P} \mathcal{V}_{n}$ и фиксированного $x \in[-1,1]$ рассмотрим полином

$$
Q(y):=P\left(\frac{1-x}{2} y+\frac{x+1}{2}\right)=c_{n}\left(\frac{1-x}{2}\right)^{n} y^{n}+\cdots .
$$

Так как для любого $y \in[-1,1]$ справедливы неравенства

$$
y \leqslant \frac{1-x}{2} y+\frac{x+1}{2} \quad \text { и }\left|\frac{1-x}{2} y+\frac{x+1}{2}\right| \leqslant 1
$$

то

$$
|Q(y) \sqrt{1+y}| \leqslant\left|P\left(\frac{1-x}{2} y+\frac{x+1}{2}\right) \sqrt{1+\left(\frac{1-x}{2} y+\frac{x+1}{2}\right)}\right| \leqslant \sqrt{2} .
$$

Таким образом, к полиному $Q(y)$ применимы теоремы 2.35 и 2.29. Согласно этим теоремам, имеем последовательно

$$
|P(x)|=|Q(-1)| \leqslant 2 n+\sqrt{\frac{\left|c_{n}(1-x)^{n}\right|}{4^{n}}} \leqslant 2 n+1 .
$$

Случай равенства проверяется непосредственно. Теорема доказана.

СлеДСтвиЕ 2.5. Для полиномов $P$ степени п с вещественными коэфбициентами имеет место неравенство

$$
\max \{|P(x)|: x \in[-1,1]\} \leqslant(2 n+1) \max \left\{\left|P(x) \sqrt{\frac{1+x}{2}}\right|: x \in[-1,1]\right\} .
$$

Равенство достигается в случае полиномов Чебыиёва третвего рода. 
ДокАЗАтЕЛьСтво. Обозначим через $M$ максимум в правой части требуемого неравенства. Полином $P / M$ принадлежит классу $\mathcal{P} \mathcal{V}_{n}$. Применяя к нему теорему 2.36, получим указанное неравенство. Для полинома Чебышёва $V_{n}$ максимум слева достигается в точке $-1,\left|V_{n}(-1)\right|=2 n+1$, а справа - в точке 1 , $\left|V_{n}(1)\right|=1$. Следствие доказано.

Полином Чебышёва $W_{n}$ является экстремальным в рассматриваемых задачах в классе $\mathcal{P} \mathcal{W}_{n}$. Действительно, если полином $P(z)$ принадлежит классу $\mathcal{P} \mathcal{W}_{n}$, то полином $P(-z)$ принадлежит классу $\mathcal{P} \mathcal{V}_{n}$. Применяя к последнему полиному предыдущие теоремы, получим соответствующие результаты в классе $\mathcal{P} \mathcal{W}_{n}$, причем экстремальным будет полином $W_{n}$, поскольку $\left|V_{n}(z)\right|=$ $\left|W_{n}(-z)\right|$ для любых $n$ и $z$. Например, из теоремы 2.29 следует, что в классе $\mathcal{P} \mathcal{W}_{n}$ полиномов $P(z)=c_{0}+\cdots+c_{n-1} z^{n-1}+c_{n} z^{n}$ справедливы точные оценки

$$
\left|c_{n}\right| \leqslant 2^{n}, \quad\left|c_{n}\right|^{2}+\left|c_{n}^{2}-2 c_{n} c_{n-1}\right| \leqslant 4^{n} .
$$

Равенство достигается в случае полинома Чебышёва $W_{n}$.

Теорема 2.32 дает неравенство

$$
\sqrt{1+x}\left|P(x)+2(x-1) P^{\prime}(x)\right| \leqslant\left(\sqrt{\frac{\left|c_{n}\right|}{2^{n}}}+2 n\right) \sqrt{2+(x-1) P^{2}(x)},
$$

справедливое для любых $x \in[-1,1]$. Равенство вновь достигается в случае полинома $W_{n}$.

С другими примерами приложений теории однолистных функций к полиномам можно ознакомиться в работах [31], [34]--[36].

\section{Глава 3. Емкости конденсаторов и симметризация}

Исследования симметризационных свойств емкостей различных типов восходят к знаменитой книге Г. Полиа и Г. Сегё [112]. Существенные приложения этих свойств в геометрической теории функций комплексного переменного даны В. К. Хейманом [113], Дж. Дженкинсом [83] и другими математиками (см. об этом, например, [37], [38]). В данной главе рассматриваются применения элементарных свойств конформной емкости конденсаторов и круговой симметризации к задачам о комплексных полиномах. Кроме того, мы приводим недавние результаты о полиномах, установленные с помощью различных обобщений и дополнений классических симметризационных принципов.

\section{1. Обобщенные конденсаторы и асимптотика их емкости при вы-} рождении пластин. Всюду ниже приняты обозначения из книги [38]. Пусть $B$ - открытое множество на комплексной плоскости $\overline{\mathbb{C}}$. Обобщенным конденсатором в $\bar{B}$ назовем тройку $C=(B, \mathcal{E}, \triangle)$, где $\mathcal{E}=\left\{E_{k}\right\}_{k=1}^{n}-$ совокупность замкнутых непустых попарно непересекающихся множеств $E_{k} \subset \bar{B}, k=1, \ldots, n$, а $\triangle=\left\{\delta_{k}\right\}_{k=1}^{n}-$ совокупность вещественных чисел $\delta_{k}, k=1, \ldots, n, n \geqslant 2$. Открытое в $\bar{B}$ множество $\bar{B} \backslash \bigcup_{k=1}^{n} E_{k}$ будем называть полем конденсатора $C$, множества $E_{k}-$ пластинами этого конденсатора, а числа $\delta_{k}-$ уровнями потенциала или, короче, потенциалами пластин $E_{k}, k=1, \ldots, n$. Емкость сар $C$ 
конденсатора $C$ определяется как точная нижняя граница интегралов Дирихле

$$
\iint_{B \backslash \bigcup_{k=1}^{n} E_{k}}\left[\left(\frac{\partial v}{\partial x}\right)^{2}+\left(\frac{\partial v}{\partial y}\right)^{2}\right] d x d y
$$

по всем допустимым функииям $v$, т. е. вещественнозначным функциям $v$, непрерывным в $\bar{B}$, удовлетворяющим условию Липшица внутри множества $B$ и равным $\delta_{k}$ на $E_{k}, k=1, \ldots, n$. В дальнейшем слово "обобщенный" в названии конденсатора будем опускать.

Одна из разновидностей монотонности емкости конденсатора состоит в следующем. Если для конденсаторов $C_{1}=\left(B_{1},\left\{E_{1 k}\right\}_{k=1}^{n}, \triangle\right)$ и $C_{2}=\left(B_{2},\left\{E_{2 k}\right\}_{k=1}^{n}, \triangle\right)$ имеют место включения $B_{1} \subset B_{2}, E_{1 k} \subset E_{2 k}, k=1, \ldots, n$, то

$$
\operatorname{cap} C_{1} \leqslant \operatorname{cap} C_{2} \text {. }
$$

Из конформной инвариантности интеграла Дирихле и граничных свойств однолистных функций вытекают различные виды конформной инвариантности емкости конденсатора $C=\left(B,\left\{E_{k}\right\}_{k=1}^{n}, \triangle\right)$. На практике в каждом конкретном случае сохранение емкости при конформном и однолистном отображении множества $B \backslash \bigcup_{k=1}^{n} E_{k}$ легко устанавливается. Поэтому мы не приводим здесь эти утверждения. Отметим лишь, что если функция $f$ конформна и однолистна на множестве $B$ и доопределена на границе $B$ в смысле граничного соответствия, то

$$
\operatorname{cap} C=\operatorname{cap} f(C),
$$

где $f(C):=\left(f(B),\left\{f\left(E_{k}\right)\right\}_{k=1}^{n}, \triangle\right)$.

Приведем один из принципов композиции конденсаторов [38]. Пусть $B_{i}$, $i=1, \ldots, m,-$ попарно непересекающиеся открытые подмножества открытого множества $B \subset \overline{\mathbb{C}}$, и пусть для конденсаторов $C_{i}=\left(B_{i},\left\{E_{i j}\right\}_{j=1}^{n_{i}},\left\{\delta_{i j}\right\}_{j=1}^{n_{i}}\right)$, $i=1, \ldots, m$, и $C=\left(B,\left\{E_{k}\right\}_{k=1}^{n},\left\{\delta_{k}\right\}_{k=1}^{n}\right)$ выполняется условие: любая пластина $E_{i j}, 1 \leqslant j \leqslant n_{i}$, каждого конденсатора $C_{i}, 1 \leqslant i \leqslant m$, принадлежит объединению пластин конденсатора $C$, имеющих тот же потенциал, что и $E_{i j}$. Тогда

$$
\sum_{i=1}^{m} \operatorname{cap} C_{i} \leqslant \operatorname{cap} C
$$

Для открытого множества $B \subset \overline{\mathbb{C}}$ обозначим $B^{+}=\{z \in B: \operatorname{Im} z>0\}$, a в случае замкнутого множества $E$ положим $E^{+}=\{z \in E: \operatorname{Im} z \geqslant 0\}$. Множество $A$ называется симметричным относительно вещественной оси, если $A=$ $\{z: \bar{z} \in A\}$. Конденсатор $C=(B, \mathcal{E}, \triangle)$ назовем симметричным относительно вещественной оси, если множество $B$ и пластины совокупности $\mathcal{E}$ обладают указанной симметрией. Имеет место следующий принцип симметрии. Если конденсатор $C=\left(B,\left\{E_{k}\right\}_{k=1}^{n},\left\{\delta_{k}\right\}_{k=1}^{n}\right)$ симметричен относительно вещественной оси, то

$$
\operatorname{cap} C=2 \operatorname{cap} C^{+},
$$

где $C^{+}=\left(B^{+},\left\{E_{k}^{+}\right\}_{k=1}^{n},\left\{\delta_{k}\right\}_{k=1}^{n}\right)$. 
Рассмотрим теперь асимптотические формулы для емкости конденсатора при вырождении некоторых из его пластин в двух важных частных случаях [38; гл. 2]. Пусть $U\left(z_{0}, r\right)=\left\{z:\left|z-z_{0}\right|<r\right\}$ в случае конечной точки $z_{0}$ и $U(\infty, r)=$ $\{z:|z|>1 / r\}$. Параметрическое семейство областей $D\left(z_{0}, r\right), 0<r<r_{0}$, назовем асимптотически круговым, если выполняются включения

$$
U\left(z_{0}, s(r)\right) \subset D\left(z_{0}, r\right) \subset U\left(z_{0}, t(r)\right)
$$

для некоторых положительных функций $s(r)$ и $t(r), 0<r<r_{0}$, удовлетворяющих условиям $s(r) \sim t(r) \sim r, r \rightarrow 0$. Элемент асимптотически кругового семейства $D\left(z_{0}, r\right)$ будем называть почти кругом с центром в точке $z_{0}$ и радиусом $r$. Пусть $B$ - открытое множество на $\overline{\mathbb{C}}, B \neq \overline{\mathbb{C}} ; Z=\left\{z_{k}\right\}_{k=1}^{n}-$ совокупность различных точек множества $B ; \triangle=\left\{\delta_{k}\right\}_{k=0}^{n}-$ совокупность вещественных чисел, $\delta_{0}=0$; и пусть $\Psi=\left\{\psi_{k}\right\}_{k=1}^{n}$, где $\psi_{k}=\psi_{k}(r) \equiv \mu_{k} r^{\nu_{k}}$, $\mu_{k}, \nu_{k}, k=1, \ldots, n,-$ произвольные положительные числа. Пусть $D\left(z_{k}, \psi_{k}(r)\right)$, $0<r<r_{0}, k=1, \ldots, n,-$ асимптотически круговые семейства областей. При достаточно малом $r>0$ определен конденсатор

$$
C(r ; B, \partial B, Z, \triangle, \Psi)=\left(B,\left\{E_{k}\right\}_{k=0}^{n}, \triangle\right),
$$

где $E_{0}=\partial B, E_{k}=\overline{D\left(z_{k}, \psi_{k}(r)\right)}, k=1, \ldots, n$. Рассмотрим асимптотику емкости этого конденсатора при $r \rightarrow 0$, т. е. когда одна пластина, $E_{0}$, фиксирована, а другие, $E_{k}$, вырождаются в точки соответственно $z_{k}, k=1, \ldots, n$. Известно, что емкость конденсатора $C(r ; B, \partial B, Z, \triangle, \Psi)$ равна

$$
Q \frac{1}{\log r}+R\left(\frac{1}{\log r}\right)^{2}+o\left(\left(\frac{1}{\log r}\right)^{2}\right), \quad r \rightarrow 0,
$$

где вещественные постоянные $Q$ и $R$ не зависят от $r$ и не зависят от выбора почти кругов. Точнее, если связные компоненты открытого множества $B$ имеют функцию Грина, то справедлива асимптотическая формула [114]:

$$
\begin{aligned}
& \operatorname{cap} C(r ; B, \partial B, Z, \triangle, \Psi)=-2 \pi \sum_{k=1}^{n} \frac{\delta_{k}^{2}}{\nu_{k} \log r} \\
& \quad+R(B, \partial B, Z, \triangle, \Psi)\left(\frac{1}{\log r}\right)^{2}+o\left(\left(\frac{1}{\log r}\right)^{2}\right), \quad r \rightarrow 0,
\end{aligned}
$$

где

$$
R(B, \partial B, Z, \triangle, \Psi)=-2 \pi\left\{\sum_{k=1}^{n} \frac{\delta_{k}^{2}}{\nu_{k}^{2}} \log \frac{r\left(B, z_{k}\right)}{\mu_{k}}+\sum_{k=1}^{n} \sum_{\substack{l=1 \\ l \neq k}}^{n} \frac{\delta_{k} \delta_{l}}{\nu_{k} \nu_{l}} g_{B}\left(z_{k}, z_{l}\right)\right\}
$$

$r\left(B, z_{k}\right)$ - внутренний радиус связной компоненты множества $B$, содержащей точку $z_{k}$, относительно этой точки, а $g_{B}\left(z, z_{k}\right)$ - функция Грина этой компоненты с полюсом в точке $z_{k}, k=1, \ldots, n$. В случае, когда множество $B$ совпадает 
со всей комплексной плоскостью $\overline{\mathbb{C}}$, пусть $Z=\left\{z_{k}\right\}_{k=1}^{n}-$ совокупность различных точек $\overline{\mathbb{C}}, \triangle=\left\{\delta_{k}\right\}_{k=1}^{n}-$ совокупность вещественных чисел и $\Psi=\left\{\psi_{k}\right\}_{k=1}^{n}$, $\psi_{k}=\psi_{k}(r) \equiv \mu_{k} r^{\nu_{k}}, \mu_{k}, \nu_{k}, k=1, \ldots, n,-$ положительные числа. Тогда

$$
\begin{aligned}
& \operatorname{cap} C(r ; \overline{\mathbb{C}}, \varnothing, Z, \triangle, \Psi)=\operatorname{cap}\left(B,\left\{E_{k}\right\}_{k=1}^{n}, \triangle\right)=-2 \pi \sum_{k=1}^{n} \frac{\left(\delta_{k}-\delta\right)^{2}}{\nu_{k} \log r} \\
& \quad+R(\overline{\mathbb{C}}, \varnothing, Z, \triangle, \Psi)\left(\frac{1}{\log r}\right)^{2}+o\left(\left(\frac{1}{\log r}\right)^{2}\right), \quad r \rightarrow 0,
\end{aligned}
$$

где

$$
\begin{aligned}
& \delta= \frac{\left(\sum_{k=1}^{n} \delta_{k} / \nu_{k}\right)}{\sum_{k=1}^{n} 1 / \nu_{k}}, \\
& R(\mathbb{C}, \varnothing, Z, \triangle, \Psi)=2 \pi\left\{\sum_{k=1}^{n} \frac{\left(\delta_{k}-\delta\right)^{2}}{\nu_{k}^{2}} \log \mu_{k}\right. \\
&\left.+\sum_{k=1}^{n} \sum_{l=1}^{n}, \frac{\left(\delta_{k}-\delta\right)\left(\delta_{l}-\delta\right)}{\nu_{k} \nu_{l}} \log \left|z_{k}-z_{l}\right|\right\}
\end{aligned}
$$

и штрих у знака суммы означает, что при $z_{k}=\infty\left(z_{l}=\infty\right)$ под соответствующим слагаемым понимается нуль [115].

3.2. Простейшие применения конформной емкости. Следующее ниже утверждение можно получить с помощью элементарных преобразований. Вместе с тем представляет интерес емкостная интерпретация этого факта.

Теорема 3.1. Для любой рациональной функции $R$ степени $n$ справедливо равенство

$$
\prod_{k=1}^{n}\left|R^{\prime}\left(a_{k}\right) R^{\prime}\left(b_{k}\right)\right|=\frac{\prod_{k=1}^{n} \prod_{\substack{l=1 \\ l \neq k}}^{n}\left|a_{k}-a_{l}\right|\left|b_{k}-b_{l}\right|}{\prod_{k=1}^{n} \prod_{l=1}^{n}\left|a_{k}-b_{l}\right|^{2}},
$$

где $a_{k}, k=1, \ldots, n,-$ корни уравнения $R(z)=0, a b_{k}, k=1, \ldots, n,-$ корни уравнения $R(z)=1$, каждый из которых считается столько раз, какова его кратность.

ДокАЗАТЕЛЬство. Можно считать, что числа $a_{k}, b_{k}, k=1, \ldots, n$, попарно различны. Положим

$$
\begin{aligned}
Z=\left\{a_{1}, \ldots, a_{n}, b_{1}, \ldots, b_{n}\right\}, & \triangle=\{1, \ldots, 1,-1, \ldots,-1\}, \quad \Psi=\left\{\psi_{k}\right\}_{k=1}^{2 n}, \\
\psi_{k}(r)=\frac{r}{\left|R^{\prime}\left(a_{k}\right)\right|}, & \psi_{k+n}(r)=\frac{r}{\left|R^{\prime}\left(b_{k}\right)\right|}, \quad k=1, \ldots, n, \\
W=\{0,1\}, & \triangle_{w}=\{1,-1\}, \quad \Psi_{w}=\{r, r\} .
\end{aligned}
$$

Так как отображение $w=R(z)$ осуществляет полное $n$-кратное накрытие сферы $\overline{\mathbb{C}}_{w}$ сферой $\overline{\mathbb{C}}_{z}$, то конформная инвариантность емкости дает

$$
\operatorname{cap} C\left(r ; \overline{\mathbb{C}}_{z}, \varnothing, Z, \triangle, \Psi\right)=n \operatorname{cap} C\left(r ; \overline{\mathbb{C}}_{w}, \varnothing, W, \triangle_{w}, \Psi_{w}\right) .
$$

Осталось воспользоваться формулой (3.5) для емкости слева и справа, где в обоих случаях $\delta=0$. Теорема доказана. 
Теорема 3.2 [22]. Пусть $B_{1}$ и $B_{2}$ - открытые непересекаюшиеся множества на сфере $\overline{\mathbb{C}}_{z}$, и пусть $P, Q$ - два полинома степени $n$, причем нули полинома $P$ (обозначим их $\left.z_{1}, \ldots, z_{n}\right)$ попарно различны и лежат в $B_{1}$, а нули полинома $Q$ (обозначим их $\left.\zeta_{1}, \ldots, \zeta_{n}\right)$ также различны и принадлежсат множеству $B_{2}$. Тогда справедливо неравенство

$$
\begin{aligned}
& \left|\prod_{k=1}^{n} \frac{P^{\prime}\left(z_{k}\right) Q^{\prime}\left(\zeta_{k}\right)}{P\left(\zeta_{k}\right) Q\left(z_{k}\right)}\right| \\
& \quad \leqslant \exp \left\{\frac{1}{2 \pi}\left[R\left(B_{1}, \partial B_{1}, Z_{1}, \triangle_{1}, \Psi_{1}\right)+R\left(B_{2}, \partial B_{2}, Z_{2}, \triangle_{2}, \Psi_{2}\right)\right]\right\}
\end{aligned}
$$

где $Z_{1}=\left\{z_{k}\right\}_{k=1}^{n}, Z_{2}=\left\{\zeta_{k}\right\}_{k=1}^{n}, \triangle_{1}=\{1, \ldots, 1\}, \triangle_{2}=\{-1, \ldots,-1\}, \Psi_{1}=\Psi_{2}=$ $\{r, \ldots, r\}$.

ДоказАтельство. Так как $B_{1} \cap B_{2}=\varnothing$, то с учетом монотонности (3.1) имеем

$$
\begin{aligned}
\operatorname{cap} & C\left(r ; B_{1}, \partial B_{1}, Z_{1}, \triangle_{1}, \Psi_{1}\right)+\operatorname{cap} C\left(r ; B_{2}, \partial B_{2}, Z_{2}, \triangle_{2}, \Psi_{2}\right) \\
& =\operatorname{cap} C\left(r ; B_{1} \cup B_{2}, \partial\left(B_{1} \cup B_{2}\right), Z_{1} \cup Z_{2}, \triangle_{1} \cup \triangle_{2}, \Psi_{1} \cup \Psi_{2}\right) \\
& \geqslant \operatorname{cap} C\left(r ; \overline{\mathbb{C}}_{z}, \varnothing, Z_{1} \cup Z_{2}, \triangle_{1} \cup \triangle_{2}, \Psi_{1} \cup \Psi_{2}\right) .
\end{aligned}
$$

Применение формул (3.4) и (3.5) дает

$$
\begin{gathered}
R\left(B_{1}, \partial B_{1}, Z_{1}, \triangle_{1}, \Psi_{1}\right)+R\left(B_{2}, \partial B_{2}, Z_{2}, \triangle_{2}, \Psi_{2}\right) \\
\geqslant 2 \pi \log \frac{\prod_{k=1}^{n} \prod_{\substack{l=1 \\
l \neq k}}^{n}\left|z_{k}-z_{l}\right|\left|\zeta_{k}-\zeta_{l}\right|}{\prod_{k=1}^{n} \prod_{l=1}^{n}\left|z_{k}-\zeta_{l}\right|^{2}}
\end{gathered}
$$

Заметим, что для полиномов $P(z)=\alpha \prod_{k=1}^{n}\left(z-z_{k}\right)$ и $Q(z)=\beta \prod_{k=1}^{n}\left(z-\zeta_{k}\right)$ выполняются равенства

$$
\begin{aligned}
& \left|\prod_{k=1}^{n} P\left(\zeta_{k}\right)\right|=|\alpha|^{n} \prod_{k=1}^{n} \prod_{l=1}^{n}\left|\zeta_{k}-z_{l}\right|, \quad\left|\prod_{k=1}^{n} Q\left(z_{k}\right)\right|=|\beta|^{n} \prod_{k=1}^{n} \prod_{l=1}^{n}\left|z_{k}-\zeta_{l}\right|, \\
& \left|\prod_{k=1}^{n} P^{\prime}\left(z_{k}\right)\right|=|\alpha|^{n} \prod_{k=1}^{n} \prod_{\substack{l=1 \\
l \neq k}}^{n}\left|z_{k}-z_{l}\right|, \quad\left|\prod_{k=1}^{n} Q^{\prime}\left(\zeta_{k}\right)\right|=|\beta|^{n} \prod_{k=1}^{n} \prod_{\substack{l=1 \\
l \neq k}}^{n}\left|\zeta_{k}-\zeta_{l}\right| .
\end{aligned}
$$

Поэтому последнее неравенство равносильно неравенству теоремы 3.2. Теорема доказана.

Выбирая в теореме 3.2 различным образом множества $B_{1}, B_{2}$ и полиномы $P, Q$, получаем различные оценки для полиномов и их производных. Например, в случае, когда $B_{1}=\{z:|z|<1\}$ и $B_{2}=\{z:|z|>1\}$, величины $R\left(B_{k}, \partial B_{k}, Z_{k}, \triangle_{k}, \Psi_{k}\right), k=1,2$, легко считаются. Поэтому из теоремы 3.2 вытекают следующие утверждения.

СлЕДСТвиЕ 3.1. Если нули полинома $P(z)=\prod_{k=1}^{n}\left(z-z_{k}\right)$ отличны от начала координат и лежат в круге $|z|<1$, а нули полинома $Q(z)=\prod_{k=1}^{n}\left(z-\zeta_{k}\right)$ лежат 
в области $|z|>1$, mо

$$
\left|\prod_{k=1}^{n} z_{k}^{n} \zeta_{k}^{n} P\left(\frac{1}{\bar{z}_{k}}\right) Q\left(\frac{1}{\bar{\zeta}_{k}}\right)\right| \leqslant\left|\prod_{k=1}^{n} P\left(\zeta_{k}\right) Q\left(z_{k}\right)\right| .
$$

Равенство достигается для произвольных полиномов $P$ и $Q$ с симметричными нулями: $\zeta_{k}=1 / \bar{z}_{k}, k=1, \ldots, n$.

СлЕДСТВИЕ 3.2. Если окружность $|z|=1$ отделяет нули $z_{1}, \ldots, z_{n}$ полинома $P(z)=z+c_{2} z^{2}+\cdots+c_{n+1} z^{n+1}, c_{n+1} \neq 0$, отличнье от начала, от нулей его производной $\zeta_{1}, \ldots, \zeta_{n}$, то

$$
\left|\prod_{k=1}^{n} P\left(\frac{1}{\bar{z}_{k}}\right) P^{\prime}\left(\frac{1}{\bar{\zeta}_{k}}\right)\right| \leqslant\left[(n+1)\left|c_{n+1}^{2}\right|\right]^{n+1}\left|\prod_{k=1}^{n} P\left(\zeta_{k}\right) P^{\prime}\left(z_{k}\right)\right| .
$$

Равенство достигается для полинома $P(z)=z-\sqrt{1 /(n+1)} z^{n+1}$.

ДоказАтельство. К полиномам $P^{\prime}(z) /\left(c_{n+1}(n+1)\right)$ и $P(z) /\left(c_{n+1} z\right)$ применимо предыдущее утверждение, из которого вытекает требуемое неравенство. Утверждение о равенстве проверяется непосредственно.

В работе [116; с. 444] установлено, что если все нули полинома $P(z)=c_{1} z+$ $\cdots+z^{n}$, отличные от $z=0$, имеют одинаковый модуль, то для критических точек $\zeta_{\nu}, \nu=1, \ldots, n-1$, выполняется неравенство

$$
\sqrt[n-1]{\prod_{\nu=1}^{n-1}\left|\frac{P\left(\zeta_{\nu}\right)}{\zeta_{\nu}}\right|} \leqslant\left|c_{1}\right| \frac{n-1}{n} .
$$

Равенство достигается для полиномов вида $P(z)=c_{1} z+z^{n}$, где $c_{1}-$ произвольное комплексное число. Ввиду (3.6) можно было бы выдвинуть более сильную гипотезу, чем следующее предположение С. Смейла [117]:

$$
\min _{1 \leqslant \nu \leqslant n-1}\left|\frac{P\left(\zeta_{\nu}\right)}{\zeta_{\nu}}\right| \leqslant\left|c_{1}\right| \frac{n-1}{n}
$$

независимо от расположения нулей полинома $P$. Однако имеет место следующий результат [13].

ТеОРема 3.3. Пусть $\zeta_{\nu}, \nu=1, \ldots, n-1,-$ критические точки полинома $P(z)=c_{1} z+\cdots+z^{n}$, и пусть все нули этого полинома, отличнье от $z=0$, расположены на $n-1$ лучах, выходящих из точки $z=0$ под равными углами величины $2 \pi /(n-1)$ так, что каждый луч содержит один и только один нуль. Тогда

$$
\sqrt[n-1]{\prod_{\nu=1}^{n-1}\left|P\left(\zeta_{\nu}\right)\right|} \geqslant(n-1)\left|\frac{c_{1}}{n}\right|^{n /(n-1)}
$$

Равенство достигается для полиномов вида $P(z)=c_{1} z+z^{n}$, где $c_{1}-$ произвольное комплексное число, отличное от нуля.

ДокАзАтЕльство. Обозначим через $z_{\mu}, \mu=1, \ldots, n-1$, нули полинома $P$, отличные от $z=0$. Так как

$$
\prod_{\nu=1}^{n-1}\left|P\left(\zeta_{\nu}\right)\right|=n^{-n}\left|c_{1}\right|^{n}\left(\prod_{\mu=1}^{n-1}\left|z_{\mu}\right|^{2-n}\right) \prod_{\substack{\mu, \nu=1 \\ \mu \neq \nu}}^{n-1}\left|z_{\nu}-z_{\mu}\right|
$$


то доказательство теоремы 3.3 сводится к установлению неравенства

$$
\prod_{\substack{\mu, \nu=1 \\ \mu \neq \nu}}^{n-1}\left|z_{\nu}-z_{\mu}\right| \geqslant(n-1)^{n-1} \prod_{\mu=1}^{n-1}\left|z_{\mu}\right|^{n-2} .
$$

Можно считать, что $\arg z_{\mu}=2 \pi(\mu-1) /(n-1), \mu=1, \ldots, n-1$. Рассмотрим конденсаторы

$$
\begin{aligned}
C(r) & =C\left(r ; \overline{\mathbb{C}}_{z}, \varnothing,\left\{0, z_{1}, \ldots, z_{n-1}\right\},\{-n+1,1, \ldots, 1\},\{r, r, \ldots, r\}\right) \\
& \equiv\left(\overline{\mathbb{C}}_{z},\left\{E_{\mu}\right\}_{\mu=0}^{n-1},\{-n+1,1, \ldots, 1\}\right), \\
C_{\mu}(r) & =\left(B_{\mu},\left\{\bar{B}_{\mu} \cap E_{0}, \bar{B}_{\mu} \cap E_{\mu}, \bar{B}_{\mu} \cap E_{\mu+1}\right\},\{-n+1,1,1\}\right),
\end{aligned}
$$

где $B_{\mu}=\{z: 2 \pi(\mu-1) /(n-1) \leqslant \arg z \leqslant 2 \pi \mu /(n-1)\}, \mu=1, \ldots, n-1\left(E_{n}=E_{1}\right)$. По принципу композиции (3.2)

$$
\operatorname{cap} C(r) \geqslant \sum_{\mu=1}^{n-1} \operatorname{cap} C_{\mu}(r) .
$$

Пусть $\zeta=f_{\mu}(z)$ - та ветвь функции $z^{(n-1) / 2}$, которая отображает область $B_{\mu}$ на верхнюю полуплоскость, и пусть $C_{\mu}^{*}(r)$ - симметричный относительно вещественной оси конденсатор, для которого $\left(C_{\mu}^{*}(r)\right)^{+}=f\left(C_{\mu}(r)\right)$. Тогда

$$
\begin{aligned}
C_{\mu}^{*}(r)=C & \left(r ; \overline{\mathbb{C}}_{\zeta}, \varnothing,\left\{0, f_{\mu}\left(z_{\mu}\right), f_{\mu}\left(z_{\mu+1}\right)\right\},\{-n+1,1,1\},\right. \\
& \left.\left\{r^{(n-1) / 2}, r\left|f_{\mu}^{\prime}\left(z_{\mu}\right)\right|, r\left|f_{\mu}^{\prime}\left(z_{\mu+1}\right)\right|\right\}\right), \quad \mu=1, \ldots, n-1 \quad\left(z_{n}=z_{1}\right) .
\end{aligned}
$$

Конформная инвариантность емкости и принцип симметрии (3.3) дают

$$
\operatorname{cap} C_{\mu}(r)=\operatorname{cap} f\left(C_{\mu}(r)\right)=\frac{1}{2} \operatorname{cap} C_{\mu}^{*}(r), \quad \mu=1, \ldots, n-1 .
$$

Окончательно,

$$
\operatorname{cap} C(r) \geqslant \frac{1}{2} \sum_{\mu=1}^{n-1} \operatorname{cap} C_{\mu}^{*}(r) .
$$

Пользуясь формулой (3.5), после несложных преобразований получаем неравенство (3.7) и, следовательно, неравенство теоремы 3.3. Случай равенства проверяется непосредственно. Теорема доказана.

СледСтвиЕ 3.3. В условиях теоремы 3.3 справедливо неравенство

$$
\sqrt[n-1]{\prod_{\nu=1}^{n-1}\left|\frac{P\left(\zeta_{\nu}\right)}{\zeta_{\nu}}\right|} \geqslant\left|c_{1}\right| \frac{n-1}{n}
$$

с равенством для полиномов $P(z)=c_{1} z+z^{n}, c_{1} \neq 0$.

Следующие два утверждения работы [19] дополняют классическое неравенство Бернштейна [2; с. 233].

ТЕОрема 3.4. Для любого полинома

$$
P(z)=c_{n} \prod_{k=1}^{n}\left(z-z_{k}\right), \quad c_{n} \neq 0,
$$


с нулями $z_{k} \in[-1,1], k=1, \ldots, n$, справедливо неравенство

$$
\sqrt[n]{\prod_{k=1}^{n}\left|P^{\prime}\left(z_{k}\right) \sqrt{1-z_{k}^{2}}\right|} \leqslant n \cdot 2^{1-n}\left|c_{n}\right| .
$$

Равенство достигается в случае полинома Чебъшёва $T_{n}$.

ДокАзАтельство. Можно считать, что все нули полинома $Р$ попарно различны и принадлежат интервалу $(-1,1)$. В книге [38; с. 150] в качестве приложения свойств емкостей конденсаторов был предложен следующий аналог классического неравенства Шура:

$$
\left(\prod_{k=1}^{n} \prod_{\substack{l=1 \\ l \neq k}}^{n}\left|z_{k}-z_{l}\right|\right) \prod_{k=1}^{n} \sqrt{1-z_{k}^{2}} \leqslant n^{n} \cdot 2^{n-n^{2}} .
$$

Остается заметить, что для любого $k=1, \ldots, n$

$$
\left|P^{\prime}\left(z_{k}\right)\right|=\left|c_{n}\right| \prod_{\substack{l=1 \\ l \neq k}}^{n}\left|z_{k}-z_{l}\right|
$$

и, следовательно,

$$
\prod_{k=1}^{n}\left|P^{\prime}\left(z_{k}\right)\right|=\left|c_{n}\right|^{n} \prod_{k=1}^{n} \prod_{\substack{l=1 \\ l \neq k}}^{n}\left|z_{k}-z_{l}\right| .
$$

Случай равенства проверяется непосредственным вычислением. Теорема доказана.

Теорема 3.5. Предположим, что полином $P$ степени $n$ удовлетворяет следующим условиям: все нули $z_{k}, k=1, \ldots, n$, полинома $P$ лежат на отрезке $[-1,1]$, а все критические значения и значения в точках $z= \pm 1$ лежат на вещественной оси вне интервала $(-1,1)$. Тогда

$$
\sqrt[n]{\prod_{k=1}^{n}\left|P^{\prime}\left(z_{k}\right) \sqrt{1-z_{k}^{2}}\right|} \geqslant n .
$$

Знак равенства выполняется в случае $P=T_{n}$.

ДокАзАТЕЛЬство. Введем следующие обозначения:

$$
B=\mathbb{C}_{w} \backslash\{w: \operatorname{Im} w=0,|\operatorname{Re} w| \geqslant 1\},
$$

$\mathscr{R}$ - риманова поверхность функции, обратной к полиному $P$, лежащая над $w$-плоскостью,

$\mathscr{P}$ - соответствующее $P$ отображение сферы $\overline{\mathbb{C}}_{z}$ на $\mathscr{R}$ (проекция $\mathscr{P}(z)$ равна $P(z))$,

$W_{k}$ - образы точек $z_{k}$ на поверхности $\mathscr{R}$ при отображении $\mathscr{P}$,

$\mathscr{B}_{k}, k=1, \ldots, n,-$ копии области $B$, лежащие на $\mathscr{R}$ над областью $B$.

Мы считаем точки $z_{k}, k=1, \ldots, n$, различными и лежащими на интервале $(-1,1)$. Из условий теоремы следует, что области

$$
D_{k}:=\mathscr{P}^{-1}\left(\mathscr{B}_{k}\right), \quad k=1, \ldots, n,
$$


являются односвязными, попарно непересекающимися и не содержащими точек $z= \pm 1$. Следовательно, дополнение

$$
\overline{\mathbb{C}}_{z} \backslash \bigcup_{k=1}^{n} D_{k}
$$

содержит некоторый континуум, соединяющий точки $z= \pm 1$. По теореме 6.18 из книги [38] справедлива оценка

$$
\prod_{k=1}^{n} \frac{r\left(D_{k}, z_{k}\right)}{\left|\sqrt{1-z_{k}^{2}}\right|} \leqslant\left(\frac{2}{n}\right)^{n}
$$

где $r(D, z)$ означает внутренний радиус области $D$ относительно точки $z[38$; c. 30]. С другой стороны,

$$
r\left(D_{k}, z_{k}\right)=r\left(\mathscr{B}_{k}, W_{k}\right) \frac{1}{\left|P^{\prime}\left(z_{k}\right)\right|}=r(B, 0) \frac{1}{\left|P^{\prime}\left(z_{k}\right)\right|}=\frac{2}{\left|P^{\prime}\left(z_{k}\right)\right|},
$$

$k=1, \ldots, n$. Осталось подставить эти соотношения в неравенство (3.8). Случай равенства проверяется непосредственным вычислением. Теорема доказана.

Неравенства для полиномов можно получить, комбинируя емкостной подход с приемами, изложенными во второй главе данного обзора. Например, исключительно из монотонности емкости (3.1) и формулы (3.4) вытекают следующие два неравенства для шварциана $S_{f}(z)$ ограниченной однолистной функции $f(z)=\alpha_{1} z+\alpha_{2} z^{2}+\alpha_{3} z^{3}+\cdots$ :

$$
\begin{gathered}
\left|S_{f}(0)\right| \leqslant 6\left(1-\left|\alpha_{1}\right|^{2}\right), \\
\left|S_{f}^{\prime \prime}(0)-S_{f}^{2}(0)\right| \leqslant 60\left(1-\left|\alpha_{1}\right|^{4}-2\left|\alpha_{2}\right|^{2}\right) .
\end{gathered}
$$

Первое неравенство восходит к 3. Нехари, а второе получено в работе [21]. Следуя главе 2, получаем теоремы 3.6 и 3.7 . Теорема 3.6. Для любах полиномов $P(z)=\sum_{k=0}^{n} c_{k} z^{k}$ степени $n$ имеют
место точные оценки

$$
\begin{aligned}
\left|c_{n}\right|^{2}+\left|c_{n} c_{n-2}\right| & \leqslant\left(\frac{H(P)-L(P)}{2}\right)^{2}, \\
\left|c_{n}\right|^{2}\left(\left|c_{n}\right|^{2}+2\left|c_{n-1}\right|^{2}\right) & \leqslant\left(\frac{H(P)-L(P)}{2}\right)^{4} .
\end{aligned}
$$

Равенство достигается для полиномов вида $P(z)=c_{0}+c_{n} z^{n}$.

ДокАЗАТЕЛЬство. Непосредственным вычислением убеждаемся, что первое неравенство вытекает из неравенства (3.9), примененного к функции, обратной функции $f$ из леммы 2.2. Второе неравенство следует из оценки (3.10), примененной к той же функции. Случай равенства очевиден. Теорема доказана.

Теорема 3.7. Для любых полиномов $P(z)=\sum_{k=0}^{n} c_{k} z^{k}$ степени $n$ с вещественными коэффициентами $c_{k}, k=0,1, \ldots, n$, имеет место точная оченка

$$
c_{n}^{2}\left(c_{n}^{2}+8 c_{n-1}^{2}\right) \leqslant 2^{4 n-8}(\bar{H}(P)-\bar{L}(P))^{4} .
$$

Равенство достигается для полинома Чебъшёва первого рода.

ДокАЗАТЕЛЬСтво вытекает из леммы 2.3 и оценки (3.10). 
3.3. Круговая симметризация. Наиболее распространенным способом симметризации множеств и конденсаторов является круговая симметризация (см., например, [83], [113], [37], [38]). Напомним определение этой симметризации открытых множеств $B$, лежащих на сфере $\overline{\mathbb{C}}_{w}$. Обозначим через $\gamma(r)$ окружность $|w|=r$ в случае $0<r<\infty$ и положим по определению окружность $\gamma(0)$ равной точке $w=0$ и $\gamma(\infty)=\infty$. Круговая симметризация относительно луча $\arg w=0$ ставит в соответствие множеству $B$ кругосимметричное множество $B^{*}$, определенное следующим образом. Если при данном $r$, $0 \leqslant r \leqslant \infty$, окружность $\gamma(r)$ не пересекается с множеством $B$, то она не пересекается и с множеством $B^{*}$. Если $\gamma(r) \subset B$, то $\gamma(r) \subset B^{*}$. В остальных случаях множество $B^{*}$ пересекается с $\gamma(r)$ по открытой дуге с центром на луче $\arg w=0$ и линейной меры, равной мере пересечения множества $B$ с $\gamma(r)$. Нетрудно видеть, что множество $B^{*}$ открыто. Аналогично определяется круговая симметризация замкнутого множества (вместо открытой дуги берем замкнутую дугу) и симметризация конденсатора с двумя пластинами в $\overline{\mathbb{C}}_{w}$ (пластины разной полярности симметризуются относительно противоположных лучей). Согласно классической теореме Г. Полиа емкость конденсатора не увеличивается при круговой симметризации (см. [112]). Этот "принцип симметризации" в сочетании с конформной инвариантностью емкости конденсатора дает многочисленные приложения в геометрической теории функций (см. [83], [113], [37]). Указанный подход распространяется на случай множеств, расположенных на некоторых римановых поверхностях [83]. Обозначим через $\mathscr{R}\left(z^{p}\right)$ риманову поверхность функции, обратной степенной функции $w=z^{p}$, где $p$ - целое число, $p>1$, и пусть $\mathscr{L}$ - один из лучей на $\mathscr{R}\left(z^{p}\right)$, лежащий над лучом $\arg w=0$. Рассмотрим произвольную компактную риманову поверхность с краем $\mathscr{R}$, лежащую над сферой $\overline{\mathbb{C}}_{w}$ и покрывающую каждую точку $w$ не более чем $p$ раз. Наконец, пусть $\mathscr{B}$ - открытое множество на поверхности $\mathscr{R}$, удовлетворяющее условию: ни для какого $r, 0<r<\infty$, не существует замкнутой жордановой кривой в $\mathscr{B}$, лежащей над окружностью $\gamma(r)$ и не покрывающей ее p-кратно. Круговая симметризация относительно луча $\mathscr{L}$ ставит в соответствие множеству $\mathscr{B}$ кругосимметричное множество $\mathscr{B}^{*} \subset \mathscr{R}\left(z^{p}\right)$, определенное следующим образом. Если при данном $r, 0 \leqslant r \leqslant \infty$, над окружностью $\gamma(r)$ нет точек множества $\mathscr{B}$, то над ней нет также и точек множества $\mathscr{B}^{*}$. Если множество $\mathscr{B}$ содержит замкнутую кривую, $p$-кратно лежащую над $\gamma(r)$, то $\mathscr{B}^{*}$ содержит замкнутую жорданову кривую, $p$-кратно лежащую над $\gamma(r)$. В остальных случаях часть множества $\mathscr{B}^{*}$, лежащая над $\gamma(r)$, представляет собой открытую жорданову дугу на $\mathscr{R}\left(z^{p}\right)$, симметричную относительно луча $\mathscr{L}$ (с центром на $\mathscr{L}$ ) и линейной меры, равной суммарной мере всех дуг на $\mathscr{B}$, лежащих над $\gamma(r)$. Определяя аналогично симметризацию замкнутых множеств на поверхности $\mathscr{R}$ и конденсаторов и следуя технике доказательств, развитой в монографии [113; гл. 4], можно получить принцип круговой симметризации Г. Полиа для емкостей конденсаторов на римановых поверхностях. Этот фундаментальный факт стандартным путем ведет к ряду утверждений в теории p-листных функций. Например, из принципа симметризации вытекает следующий результат (см. [113; теорема 5.1]). Предположим, что голоморфная и р-листная в круге $|z|<1$ функция

$$
f(z)=a_{0}+a_{1} z+\cdots
$$


не принимает значение $w=0$ в этом круге. Тогда

$$
\left|a_{1}\right| \leqslant 4 p\left|a_{0}\right| \text {. }
$$

В тех случаях, когда задачу о полиномах удается свести к задаче для однолистных функций, возможны прямые применения круговой симметризации (см., например, доказательство теоремы 3.15). В произвольном случае требуется модификация этой симметризации для множеств, лежащих на римановых поверхностях. Заметим, что прямое применение теорем из монографий [113], [83] к полиномам степени $n$ (как к $n$-листным функциям) дает неточные либо тривиальные оценки. Вместе с тем, совершенствуя технику симметризации и диссимметризации множеств на римановых поверхностях, можно получить существенные результаты для полиномов. В разделах 3.4-3.6 приводятся теоремы, доказанные таким путем. Ввиду сложности некоторых доказательств мы опускаем их в данной обзорной статье. Круговая симметризация допускает также модификацию, когда результат симметризации расположен на римановой поверхности функции, обратной полиному Чебышёва первого рода [21].

3.4. K теоремам о конечном приращении. Общая теорема о конечном приращении дифференцируемых отображений в случае комплексных полиномов имеет вид

$$
\left|P\left(z_{1}\right)-P\left(z_{2}\right)\right| \leqslant\left|P^{\prime}(\xi)\right|\left|z_{1}-z_{2}\right|,
$$

где $z_{1}$ и $z_{2}$ - произвольные комплексные числа, а $\xi$ - некоторая точка отрезка $\left[z_{1}, z_{2}\right]$. Естественно поставить вопрос об уточнении и дополнении неравенства (3.12) с использованием специфики отображения, осуществляемого полиномом $P$. В частности, для каких точек $z_{1}$ и $z_{2}$ справедливо неравенство (3.12) с возможным положительным множителем в правой части, зависящим только от степени $n$, если точка $\xi$ является концом отрезка $\left[z_{1}, z_{2}\right]$ ? Имеет ли место неравенство, противоположное неравенству (3.12) и также с некоторым положительным множителем в правой части? Несмотря на обилие литературы по полиномам, указанный вопрос мало изучен (см., например, [1]-[3]). Отметим аналог неравенства (3.12) в случае, когда одна из точек $z_{k}$ является критической:

$$
|P(z)-P(\zeta)| \leqslant 4\left|P^{\prime}(z)\right||z-\zeta| .
$$

Здесь $z$ - произвольная некритическая точка полинома $P$, а $\zeta$ - некоторая его критическая точка. Доказательство выписанного неравенства получено С. Смейлом [117], который предположил также, что константу 4 можно заменить на 1 либо $1-1 / n[3 ; \S 7.2]$. В работе [26] поставлен вопрос о получении противоположного неравенства (с другой константой) и, в частности, установлено, что для некоторой критической точки $\zeta$ справедлива оценка

$$
|P(z)-P(\zeta)| \geqslant\left|\frac{P^{\prime}(z)}{n \cdot 4^{n}}\right||z-\zeta| .
$$

В [26] выдвигается гипотеза, что константу $n \cdot 4^{n}$ в неравенстве (3.13) можно заменить на $n$.

Следующее утверждение рассматривается как первый шаг в получении нижних оценок модуля приращения полинома для произвольных точек комплексной плоскости (не обязательно критических). 
Теорема 3.8. Для любого полинома $P$ степени не выше $n$ и любых точек $z_{1}$ и $z_{2}$ комплексной плоскости $\mathbb{C}$ существует точка $\zeta \in \mathbb{C}$ такая, что $P(\zeta)=P\left(z_{2}\right)$ и выполняется неравенство

$$
\left|P\left(z_{1}\right)-P\left(z_{2}\right)\right| \geqslant \frac{4^{1 / n-1}}{n}\left|P^{\prime}\left(z_{1}\right)\right|\left|z_{1}-\zeta\right| .
$$

В общем случае точку $\zeta$ в неравенстве (3.14) нельзя заменить на $z_{2}$. Например, для полинома

$$
P(z)=\frac{1-(1-z)^{n}}{n}=z+c_{2} z^{2}+\cdots,
$$

точки $z_{1}=0$ и для точек $z_{2}$, достаточно близких к корням $P$, отличным от $z_{1}$, величина $\left|P\left(z_{2}\right) / z_{2}\right|$ принимает сколь угодно малые значения $\left(<4^{1 / n-1} / n\right)$. Доказательство теоремы 3.8 опирается на неравенство (3.11) и свойства диссимметризации областей [37]. Ранее диссимметризацию в оценках для полиномов применяли в работе [118]. Интересно отметить, что для полинома $P$ степени $n$ и произвольных точек $z_{1}, z_{2}$ легко устанавливается существование точки $\zeta$ такой, что $P(\zeta)=P\left(z_{1}\right)$ и выполняется неравенство

$$
\left|P\left(z_{1}\right)-P\left(z_{2}\right)\right| \geqslant\left|\frac{P^{\prime}(\zeta)}{n}\right| \zeta-z_{2} \mid .
$$

Действительно, считая корни $\zeta_{k}, k=1, \ldots, n$, уравнения $P(\zeta)=P\left(z_{1}\right)$ различными, имеем для любых $z \in \mathbb{C}$

$$
1=\left|\sum_{k=1}^{n} \frac{P(z)-P\left(z_{1}\right)}{P^{\prime}\left(\zeta_{k}\right)\left(z-\zeta_{k}\right)}\right| \leqslant \sum_{k=1}^{n}\left|\frac{P(z)-P\left(z_{1}\right)}{P^{\prime}\left(\zeta_{k}\right)\left(z-\zeta_{k}\right)}\right|,
$$

что дает требуемое неравенство при некотором $\zeta=\zeta_{k}, 1 \leqslant k \leqslant n$. Равенство достигается в случае полинома $P(z)=1+z^{n}$ и точек $\zeta=z_{1}=1, z_{2}=0$.

Известная теорема покрытия Кёбе-Бибербаха утверждает, что если функция $f(z)=z+c_{2} z^{2}+\cdots$ голоморфна и однолистна в круге $|z|<1$, то образ круга $|z|<1$ при отображении этой функцией целиком покрывает круг $|w|<1 / 4$, т. е. круг один и тот же для всех функций такого рода. Соответствующая теорема для всего класса функций $f(z)=z+c_{2} z^{2}+\cdots$, голоморфных в $|z|<1$, не верна, т. е. нельзя указать круга абсолютного радиуса и с центром в начале, который покрывался бы целиком образом круга $|z|<1$ при отображении каждой такой функцией. Тем не менее из теоремы 3.8 непосредственно вытекает следующее утверждение.

СлеДСТВИЕ 3.4. Для любого вещественного числа $R>0$ образ круга $|z|<R$ при отображении полиномом $P(z)=z+c_{2} z^{2}+\cdots$ степени не выше $n$ иеликом покрывает круг $|w|<R \cdot 4^{1 / n-1} / n$.

Как показывает пример приведенного выше полинома $P$, указанное в следствии 3.4 накрытие не обязано быть $n$-кратным. Интересно было бы найти радиус наибольшего круга в следствии 3.4 , покрываемого кругом $|z|<R$ при отображении $P$.

Теорема 3.9. Пусть $P$ - полином степени не выше $n, z_{1} u z_{2}$ - произвольные точки комплексной плоскости $\mathbb{C}$, и пусть $\gamma$ - связная компонента 
прообраза отрезка $\left[P\left(z_{1}\right), P\left(z_{2}\right)\right]$ при отображении $P$, содержащая точку $z_{1}$. Тогда для любой точки $\zeta \in \gamma$ выполняется неравенство

$$
\left|P\left(z_{1}\right)-P\left(z_{2}\right)\right| \geqslant \frac{1}{n^{2}}\left|P^{\prime}\left(z_{1}\right)\right|\left|z_{1}-\zeta\right| .
$$

Равенство в (3.15) достигается для полинома Чебышёва $T_{n}$ и точек $z_{1}=1$, $\zeta=-1$ и для произвольной точки $z_{2}$, удовлетворяющей условию $T_{n}\left(z_{2}\right)=-1$.

Из определения полинома $T_{n}$ легко следует равенство в соотношении (3.15) для этого полинома и точек, указанных в теореме 3.9. Доказательство теоремы 3.9 получено методом симметризации [18] и восходит в идейном плане к доказательству гипотезы В. К. Хеймана о покрытии вертикальных отрезков при конформном отображении круга (см. [119]). В случае, когда континуум $\gamma$ в теореме 3.9 есть отрезок, неравенство (3.15) вытекает из классического неравенства Маркова. Немного сложнее увидеть, что неравенство Маркова содержится в теореме 3.9. Действительно, можно считать, что полином $P$ имеет вещественные коэффициенты, $P([-1,1])=[-1,1]$ и $P( \pm 1)= \pm 1$. Применяя теорему 3.9 к подходящему континууму из $P^{-1}([-1, P(x)])$ либо $P^{-1}([P(x), 1])$, получим неравенство Маркова

$$
\left|P^{\prime}(x)\right| \leqslant n^{2},
$$

где $\left|P^{\prime}(x)\right|=\max \left\{\left|P^{\prime}(z)\right|: z \in[-1,1]\right\},-1 \leqslant x \leqslant 1$. Можно показать, что из теоремы 3.9 следует усиление неравенства (3.13) с заменой константы $n \cdot 4^{n}$ на $n^{2}$. Сравнительно недавно А. Еременко [120] установил замечательное неравенство марковского типа для произвольного континуума, из которого вытекает, в частности, ослабленная версия неравенства (3.15) с дополнительным множителем $2^{1 / n}$ в его левой части, а также неравенство (3.14) теоремы 3.8 с меньшей константой в правой части.

ДокаЗАТЕЛЬСтво теоремы 3.8. Можно считать, что степень полинома $P$ равна $n, z_{1} \neq z_{2}$ и $P^{\prime}\left(z_{1}\right) \neq 0$. Линейной заменой общий случай сводится к ситуации, когда $z_{2}=P\left(z_{2}\right)=0$ и, следовательно, неравенство (3.14) имеет вид

$$
\left|P\left(z_{1}\right)\right| \geqslant \frac{4^{1 / n-1}}{n}\left|P^{\prime}\left(z_{1}\right)\right|\left|z_{1}-\zeta\right| .
$$

Предположим, что неравенство $\left(3.14^{\prime}\right)$ не выполняется ни при каких $\zeta, P(\zeta)=$ 0 , и пусть $\zeta_{1}, \ldots, \zeta_{m}$ - все различные корни полинома $P, m \leqslant n$. Введем следующие обозначения:

- пусть

$$
\alpha=\min _{1 \leqslant k \leqslant m}\left|z_{1}-\zeta_{k}\right|>\frac{n\left|P\left(z_{1}\right)\right|}{4^{1 / n-1}\left|P^{\prime}\left(z_{1}\right)\right|} ;
$$

- $D$ - плоскость $\mathbb{C}$ с разрезами вдоль лучей

$$
\left\{z: \arg \left(z-z_{1}\right)=\arg \left(\zeta_{k}-z_{1}\right),\left|z-z_{1}\right| \geqslant \alpha\right\}, \quad k=1, \ldots, m ;
$$

- $\varphi$ - функция, конформно и однолистно отображающая круг $|\omega|<1$ на область $D$ так, что $\varphi(0)=z_{1}, \varphi^{\prime}(0)>0$;

- $D^{*}$ - плоскость $\mathbb{C}$ с разрезами вдоль лучей

$$
\left\{z: \arg \left(z-z_{1}\right)=\frac{2 \pi(k-1)}{n},\left|z-z_{1}\right| \geqslant \alpha\right\}, \quad k=1, \ldots, n
$$


- $\varphi^{*}$ - функция, конформно и однолистно отображающая круг $|\omega|<1$ на $D^{*}, \varphi^{*}(0)=z_{1}, \varphi^{* \prime}(0)>0$.

По лемме 1.4 работы [37] существует диссимметризация области $D^{*}$ в подмножество $D$. Ввиду следствия 1.4 этой работы, получаем, что

$$
\left|\varphi^{\prime}(0)\right| \geqslant\left|\varphi^{* \prime}(0)\right|=\alpha \cdot 4^{1 / n} .
$$

Из неравенства (3.11), примененного к суперпозиции $P(\varphi(\omega))$, вытекает оценка

$$
\left|P^{\prime}\left(z_{1}\right) \varphi^{\prime}(0)\right| \leqslant 4 n\left|P\left(z_{1}\right)\right|,
$$

которая вместе с (3.16) и (3.17) приводит к противоречию. Теорема доказана.

3.5. О свойствах полиномов внутри лемнискаты. Всюду ниже

$$
P(z)=\prod_{\mu=1}^{n}\left(z-z_{\mu}\right),
$$

где $n \geqslant 2$ и $z_{\mu}, \mu=1, \ldots, n,-$ комплексные числа. Множество

$$
E(P)=\{z:|P(z)| \leqslant 1\}
$$

принято называть лемнискатой [2]. Метрическим свойствам лемнискаты, а также взаимному расположению нулей $z_{\mu}$ и критических точек полинома $P$ (т. е. точек, где $P^{\prime}=0$ ) посвящено немало работ, а некоторые задачи в данной тематике не решены до сих пор (см., например, [2], [3]). Весьма полезным при изучении свойств лемнискаты является понятие логарифмической емкости континуума (далее - емкости) [121]. Ниже устанавливается емкостное неравенство, характеризующее в некоторой степени разброс нулей и критических точек полинома $P$ внутри лемнискаты $E(P)$. С другой стороны, если лемниската известна, то это неравенство можно трактовать как оценку емкости континуума, проходящего через заданные точки. Предположим, что

$$
\max \left\{|P(\zeta)|: P^{\prime}(\zeta)=0\right\} \leqslant 1 .
$$

Тогда множество $E(P)$ связно, и поэтому следующее утверждение верно с константой $\sigma=1$ : для любых точек $a$ и $b$ лемнискаты $E(P)$ существует континуум $\gamma \subset E(P)$ емкости сар $\gamma \leqslant \sigma$, содержащий точки $a, b$, а также все нули и все критические точки полинома $P$. В роли $\gamma$ в этом случае выступает само множество $E(P)$. Естественно поставить вопрос о наименьшем значении величины $\sigma$, при котором указанное выше утверждение имеет место для любых полиномов (3.18). Заметим, что условие (3.19) в такой постановке является необходимым, ибо в противном случае множество $E(P)$ несвязно. Справедлива следующая теорема [14].

ТЕОрема 3.10. Пусть полином (3.18) удовлетворяет условию (3.19), и пусть $m$ - число всех критических точек этого полинома. Тогда для любых $n-m+1$ точек лемнискаты $E(P)$ существует континуум $\gamma \subset E(P)$ емкости

$$
\operatorname{cap} \gamma \leqslant 2^{-1 / n}
$$

содержащий эти точки, а также все нули и критические точки полинома $P$. 
Число “свободных" точек лемнискаты $E(P)$ в оценке (3.20) не меньше двух. Поэтому независимо от числа критических точек для любых двух точек лемнискаты всегда найдется континуум $\gamma \subset E(P)$, удовлетворяющий (3.20) и соединяющий эти точки с нулями и критическими точками полинома $P$. Пример полинома $P(z)=T_{n}\left(2^{(1-n) / n} z\right)$, где $T_{n}(z)=2^{n-1} z^{n}+\ldots-$ полином Чебышёва, двух точек $\pm 2^{1-1 / n}$ и континуума $\gamma=\left[-2^{1-1 / n}, 2^{1-1 / n}\right]$ показывает, что постоянная в правой части неравенства (3.20) не может быть улучшена. Наибольшее число "свободных" точек равно $n$, и в этом случае необходимо $m=1$, a $P(z)=\left(z+d_{1}\right)^{n}+d_{2}$, где $d_{1}, d_{2}-$ комплексные числа. Полагая для определенности $d_{1}=0, d_{2}=-1$, легко заключаем, что равенство в (3.20) достигается для полинома $P(z)=z^{n}-1$, точек $\sqrt[n]{2} \exp (2 \pi i j / n), j=1, \ldots, n$, и континуума $\gamma=\left\{z:|z| \leqslant \sqrt[n]{2}, \arg z^{n}=0\right\}$ (о вычислении емкости см. [121; гл. II, п. 17]). Этими примерами не исчерпываются все случаи равенства в (3.20). Отметим, что из неравенства (3.20) вытекает известная оценка сверху для диаметра лемнискаты $E(P)$ в случае, когда полином $P$ удовлетворяет условию (3.19):

$$
\operatorname{diam} E(P) \leqslant 2^{2-1 / n}
$$

(см. [122]).

ДОКАЗАТЕЛЬСтво теОРемЫ 3.10. Обозначим через $\zeta_{\nu}, \nu=1, \ldots, m$, все различные критические точки полинома $P$, и пусть $a_{j}, j=1, \ldots, n-m+1,-$ произвольные точки лемнискаты $E(P)$ из условия теоремы. Пусть $\mathscr{P}$ - аналитическая функция, обратная функции $w=P(z)$, и пусть $\mathscr{R}$ - риманова поверхность функции $\mathscr{P}[68]$. Далее рассматриваем функцию $\mathscr{P}$ как однозначную функцию, заданную на поверхности $\mathscr{R}$. Под проекцией точки $W \in \mathscr{R}$ понимается точка $P(\mathscr{P}(W)) \in \overline{\mathbb{C}}_{w}$. Отрезком на $\mathscr{R}$ назовем любую жорданову кривую на поверхности $\mathscr{R}$, однолистно лежащую над некоторым отрезком $w$-плоскости. Аналогично определяется луч на $\mathscr{R}$ - как жорданова кривая, однолистно лежащая над некоторым лучом вида $\arg w=$ const, $\rho \leqslant|w| \leqslant \infty$. Обозначим через $\mathscr{L}$ объединение всех отрезков на $\mathscr{R}$, у которых начало расположено над точкой $w=0$, а конец совпадает с конечной точкой ветвления поверхности $\mathscr{R}$ (т. е. с одной из точек $\left.\mathscr{P}^{-1}\left(\zeta_{\nu}\right), \nu=1, \ldots, m\right)$. В случае нулевых критических значений некоторые из этих отрезков могут оказаться вырожденными. Покажем, что множество $\mathscr{L}$ связно и каждая точка поверхности $\mathscr{R}$, лежащая над $w=0$, принадлежит $\mathscr{L}$. Для этого разобьем поверхность $\mathscr{R}$ лучами, соединяющими конечные точки ветвления $\mathscr{R}$ с бесконечностью, на $n$ простых листов (далее - листов) $L_{k}, k=1, \ldots, n$. Каждый лист $L_{k}$ есть плоскость с одним или, возможно, большим числом разрезов, $k=1, \ldots, n$. Поэтому каждый лист $L_{k}$ содержит по крайней мере один отрезок (возможно вырожденный), принадлежащий $\mathscr{L} \cap \bar{L}_{k}$, и, следовательно, множество $\mathscr{L}$ покрывает $n$-кратно точку $w=0$. Здесь $\bar{L}_{k}$ означает замыкание $L_{k}$ в $\mathscr{R}$. Заметим, что для любого $k$, $1 \leqslant k \leqslant n$, множество $\mathscr{L} \cap \bar{L}_{k}$ связно. Склеивая данный лист $L_{k^{\prime}}$ по одному из берегов разреза с другим листом $L_{k^{\prime \prime}}$ так, как это было на поверхности $\mathscr{R}$, получаем связное множество $\mathscr{L} \cap\left(\bar{L}_{k^{\prime}} \cup \bar{L}_{k^{\prime \prime}}\right)$. Затем к множеству $\bar{L}_{k^{\prime}} \cup \bar{L}_{k^{\prime \prime}}$ приклеиваем другой лист $L_{k^{\prime \prime \prime}}$, получая связное множество $\mathscr{L} \cap\left(\bar{L}_{k^{\prime}} \cup \bar{L}_{k^{\prime \prime}} \cup \bar{L}_{k^{\prime \prime \prime}}\right)$ и так далее, пока склеенные листы не образуют всю поверхность $\mathscr{R}$. Отсюда вытекает связность множества $\mathscr{L}$ и, как следствие, односвязность области $\mathscr{R} \backslash \mathscr{L}$. Обозначим через $f$ одну из регулярных ветвей аналитической функции 
$\omega=(P(z))^{1 / n}$ в односвязной области $B:=\mathscr{P}(\mathscr{R} \backslash \mathscr{L})$. Функция $f$ конформно и однолистно отображает область $B$ на область $f(B)$, представляющую собой $\omega$-плоскость, разрезанную по прямолинейным отрезкам. Каждый такой отрезок соединяет точку $\omega=0$ с точкой, модуль которой больше нуля, но не превосходит единицы. Число указанных отрезков не превосходит $n+m-1$. Действительно, каждая точка ветвления порождает $n_{\nu}+1$ отрезок, где $n_{\nu}-$ кратность нуля $\zeta_{\nu}$ производной полинома $P$, и некоторые из этих отрезков вырожденные, а

$$
\sum_{\nu=1}^{m}\left(n_{\nu}+1\right)=n-1+m .
$$

Отсюда следует, что континуум

$$
\Gamma=\left(\mathbb{C}_{\omega} \backslash f(B)\right) \cup\left(\bigcup_{j=1}^{n-m+1}\left[0, f\left(a_{j}\right)\right]\right)
$$

состоит из не более чем $2 n$ невырожденных отрезков, выходящих из начала, длины которых не превосходят единицы. Введем также симметричный континуум

$$
\Gamma^{*}=\left\{\omega:|\omega| \leqslant 1, \arg \omega^{2 n}=0\right\},
$$

состоящий из $2 n$ отрезков. Емкость сар $\Gamma^{*}$ равна $2^{-1 / n}[121 ;$ гл. II, п. 17]. Из монотонности емкости и следствия 1.4 работы [37] имеем

$$
2^{-1 / n}=\operatorname{cap} \Gamma^{*} \geqslant \operatorname{cap} \Gamma \text {. }
$$

Осталось рассмотреть континуум

$$
\gamma=\mathscr{P}(\mathscr{L}) \cup f^{-1}\left(\bigcup_{j=1}^{n-m+1}\left[0, f\left(a_{j}\right)\right]\right),
$$

соединяющий точки $a_{j}, j=1, \ldots, n-m+1$, а также все нули и критические точки полинома $P$. Поскольку

$$
f\left(\overline{\mathbb{C}}_{z} \backslash \gamma\right)=\overline{\mathbb{C}}_{\omega} \backslash \Gamma
$$

и разложение функции $f$ в окрестности бесконечности имеет вид

$$
f(z)=\alpha z+\cdots, \quad|\alpha|=1,
$$

Tо

$$
\operatorname{cap} \Gamma=\operatorname{cap} \gamma
$$

Теорема доказана.

Хорошо известна задача Чеботарёва о континууме $\gamma\left(c_{1}, \ldots, c_{l}\right)$ наименьшей емкости, содержащем заданные точки $c_{1}, \ldots, c_{l}$ плоскости $\mathbb{C}_{z}[123 ; \S \S 4,5]$. С этой задачей связаны многие экстремальные вопросы теории однолистных функций. Вместе с тем ее решение при $n \geqslant 4$ до сих пор неизвестно. Даже в случае решения задачи Чеботарёва емкость экстремального континуума наверняка будет иметь сложное аналитическое представление. Неравенство (3.20) позволяет оценить сар $\gamma\left(c_{1}, \ldots, c_{l}\right)$ сверху в ряде частных случаев. Рассмотрим некоторые из них. 
СлеДСТВИЕ 3.5. Для заданнъх точек $z_{\mu} \in \mathbb{C}_{z}, \mu=1, \ldots, n$, обозначим через $M$ величину $\max \left\{|P(\zeta)|: P^{\prime}(\zeta)=0\right\}$, где $P-$ полином (3.18). Тогда для любых двух точек $a_{1}$ и $a_{2}$, удовлетворяющих условию $\left|P\left(a_{j}\right)\right| \leqslant M, j=1,2$, справедливо неравенство

$$
\operatorname{cap} \gamma\left(z_{1}, \ldots, z_{n}, a_{1}, a_{2}\right) \leqslant\left(\frac{M}{2}\right)^{1 / n} .
$$

Равенство достигается, например, в случае, когда $z_{\mu} \cdot 2^{1 / n-1}, \mu=1, \ldots, n,-$ нули полинома Чебышёва $T_{n}$ и $a_{j}=(-1)^{j} \cdot 2^{1-1 / n}, j=1,2$.

В связи с последним неравенством представляется интересным установить оценку сверху для величины $\max \left\{|P(\zeta)|: P^{\prime}(\zeta)=0\right\}$ в случае, когда все нули $z_{\mu}$, $\mu=1, \ldots, n$, полинома $P(z)=z^{n}+\cdots$ лежат в некотором круге $|z| \leqslant R$. Заметим, что в этом случае из свойств диссимметризации [37] легко следует оценка

$$
\operatorname{cap} \gamma\left(z_{1}, \ldots, z_{n}\right) \leqslant 2^{-2 / n} R
$$

с равенством для континуума $\gamma\left(z_{1}^{*}, \ldots, z_{n}^{*}\right)=\left\{z:|z| \leqslant R, \arg z^{n}=0\right\}$, где $z_{\mu}^{*}=R \exp (2 \pi i \mu / n), \mu=1, \ldots, n$.

Широта применения следствия 3.5 ограничена сложностью вычисления константы $M$. Однако теорема 3.10 дает также другой подход к оценкам наименьшей емкости.

СлеДСТВиЕ 3.6. Для заданнъх точек $\zeta_{\nu} \in \mathbb{C}_{z}, \nu=1, \ldots, n-1$, пусть

$$
P(z)=n \int_{0}^{z}\left(z-\zeta_{1}\right) \cdots\left(z-\zeta_{n-1}\right) d z+d_{0}=z^{n}+\cdots,
$$

где $d_{0}$ - некоторое комплексное число. Тогда для любых точек $a_{1}$ и $a_{2}$, удовлетворяющих условию

$$
\left|P\left(a_{j}\right)\right| \leqslant M:=\max _{1 \leqslant \nu \leqslant n-1}\left|P\left(\zeta_{\nu}\right)\right|
$$

справедливо неравенство

$$
\operatorname{cap} \gamma\left(\zeta_{1}, \ldots, \zeta_{n-1}, a_{1}, a_{2}\right) \leqslant\left(\frac{M}{2}\right)^{1 / n} .
$$

Равенство имеет место, например, в случае, когда $\zeta_{\nu} \cdot 2^{1 / n-1}, \nu=1, \ldots, n-1,-$ нули производной полинома Чебышёва $T_{n}, d_{0}=T_{n}(0)$ u $a_{j}=(-1)^{j} \cdot 2^{1-1 / n}$, $j=1,2$.

Пусть $R$ - рациональная функция степени $n$, представимая в виде $R=P / Q$, где $P$ и $Q$ - полиномы, не имеющие общих нулей, причем $P$ - полином степени $n$, а $Q$ - полином степени $<n$, и пусть $R(0)=0, R^{\prime}(0) \neq 0$. Т. Шейл-Смолл поставил вопрос об определении хотя бы какой-нибудь окрестности точки $w=$ 0 , в которой можно было бы выделить однозначную ветвь $f(w)$ обратной функции $z=R^{-1}(w), f(0)=0$, удовлетворяющую неравенству

$$
\operatorname{Re} \frac{w f^{\prime}(w)}{f(w)} \geqslant \frac{1}{n}
$$

(см. [4; п. 10.3.2]). Полученное неравенство представляло бы интерес в связи с изучением формы линий уровня $|R(z)|=$ const в области однолистности функции $R$. Рассмотрим близкую задачу, но только для полиномов $P(Q \equiv 1)$. 
ТеОрема 3.11 [17]. Пусть $P$ - полином степени не выше $n$. Предположим, что связная компонента $E$ лемнискаты $|P(z)| \leqslant t, t>0$, не содержит критических точек этого полинома, отличных от его нулей. Тогда для любой точки $z \in E \backslash\{a\}$ выполняется неравенство

$$
\left|\frac{(z-a) P^{\prime}(z)}{P(z)}\right| \leqslant n,
$$

где а - нуль полинома $P$, принадлежащий компоненте E. Равенство в (3.21) для любой точки z достигается в случае $P(z)=c z^{n}, c \neq 0$.

Если компонента $E$ не содержит критических точек, то из (3.21) следует, что для соответствующей ветви $f$ обратной к полиному $P$ функции в круге $|w|<t$ имеет место неравенство

$$
\left|\frac{w f^{\prime}(w)}{f(w)-a}\right| \geqslant \frac{1}{n} .
$$

Это слабее постановки Шейл-Смолла, однако в отличие от [4] мы допускаем наличие критической точки в $E$. Неравенство (3.21) имеет следующее геометрическое истолкование. Пусть для простоты $a=0$ и $\log (\cdot)$ означает однозначную ветвь логарифма, отображающую плоскость с разрезом вдоль вещественной положительной полуоси на некоторую полосу ширины $2 \pi$. Для любой линии уровня $c(\tau)(|P(z)|=\tau<t)$ "кривая" $\gamma(\tau)=\log c(\tau)$ соединяет противоположные стороны указанной полосы, и, следовательно, ее длина не меньше $2 \pi$. С другой стороны, образ $\gamma(\tau)$ при отображении $\log P(\exp (\cdot))$ покрывает вертикальный отрезок длины $2 \pi$ не более чем $n$ раз. Следовательно, на кривой $\gamma(\tau)$ найдется точка $\zeta$, в которой коэффициент растяжения не превосходит $n$ :

$$
\left|[\log P(\exp \zeta)]^{\prime}\right| \leqslant n,
$$

что означает неравенство (3.21) в некоторой точке $z=\exp \zeta$ линии уровня $c(\tau)$. Теорема 3.11 утверждает, что на самом деле это неравенство имеет место в любой точке кривой $c(\tau)$. Требование, чтобы множество $E$ не содержало критической точки, отличной от нуля, является существенным. Например, для полинома $P(z)=z^{3} / 2-3 z^{2} / 4$ лемниската $|P(z)| \leqslant 1$ содержит обе критические точки $z=0$ и $z=1$ и, следовательно, является связной. Точка $z=2$ принадлежит этой лемнискате, но

$$
\frac{2 P^{\prime}(2)}{P(2)}=6>3 .
$$

СледСтвиЕ 3.7. Если в условиях теоремы 3.11 в некоторой точке $z \in E$ выполняется неравенство $P(z)>0$, то для полярной производной $D_{a} P$ относительно точки а справедлива ощенка

$$
\operatorname{Re} D_{a} P(z)=\operatorname{Re}\left[n P(z)-(z-a) P^{\prime}(z)\right] \geqslant 0 .
$$

Доказательство теоремы 3.11 получено методом симметризации (см. [17]).

3.6. Неравенства для критических значений полиномов. Всюду в дальнейшем $P$ означает алгебраический полином степени $n, n \geqslant 2$. Точка $\zeta$ называется критической точкой полинома $P$, если $P^{\prime}(\zeta)=0$. Под критическим значением полинома $P$ понимается величина $P(\zeta)$, где $\zeta$ - критическая 
точка $P$. Несмотря на простоту этих понятий имеется много нерешенных проблем, связанных с критическими точками и критическими значениями полиномов (см., например, монографии [1]-[3]). Приведенные ниже точные оценки средних геометрических модулей критических значений полезны при изучении свойств таких значений в зависимости от расположения нулей исходного полинома. Доказательство следующего утверждения можно отнести к фольклору.

Теорема 3.12. Если все нули полинома $P(z)=c_{0}+\cdots+z^{n}$ лежат в единичном круге $|z| \leqslant 1$, то для критических точек $\zeta_{k}, k=1, \ldots, n-1$, этого полинома (пронумерованных с учетом кратности) справедливо неравенство

$$
\sqrt[n-1]{\prod_{\nu=1}^{n-1}\left|P\left(\zeta_{\nu}\right)\right|} \leqslant 1
$$

Равенство достигается в случае $P(z)=c_{0}+z^{n},\left|c_{0}\right|=1$.

ДокАзАТЕльство. Обозначим через $z_{\mu}, \mu=1, \ldots, n$, нули полинома $P$, также пронумерованные с учетом их кратности. Сравнивая разложения

$$
P(z)=\prod_{\mu=1}^{n}\left(z-z_{\mu}\right) \quad \text { и } \quad P^{\prime}(z)=n \prod_{\mu=1}^{n-1}\left(z-\zeta_{\mu}\right)
$$

и используя равенство

$$
P^{\prime}\left(z_{\nu}\right)=\prod_{\substack{\mu=1 \\ \mu \neq \nu}}^{n}\left(z_{\nu}-z_{\mu}\right)
$$

приходим к следующим соотношениям:

$$
n^{n} \prod_{\nu=1}^{n-1}\left|P\left(\zeta_{\nu}\right)\right|=\prod_{\nu=1}^{n}\left|P^{\prime}\left(z_{\nu}\right)\right|=\prod_{\substack{\mu, \nu=1 \\ \mu \neq \nu}}^{n}\left|z_{\nu}-z_{\mu}\right| .
$$

Осталось исследовать на максимум величину

$$
\prod_{\substack{\mu, \nu=1 \\ \mu \neq \nu}}^{n}\left|z_{\mu}-z_{\nu}\right|
$$

при всевозможных значениях $z_{\mu},\left|z_{\mu}\right| \leqslant 1, \mu=1, \ldots, n$. Из принципа максимума модуля для аналитических функций следует, что этот максимум достигается в случае $\left|z_{\mu}\right|=1, \mu=1, \ldots, n$. Классическое неравенство Шура [124] показывает, что наибольшее значение реализуется в случае $n$-кратно симметричной системы точек $\left\{z_{\mu}\right\}_{\mu=1}^{n}$ и равно $n^{n}$ (этот факт вытекает также из свойств диссимметризации конденсаторов [38]). Сказанное выше ведет к требуемому неравенству. Непосредственно убеждаемся, что равенство достигается для полиномов вида $P(z)=c_{0}+z^{n}$, где $c_{0}$ - комплексное число с $\left|c_{0}\right|=1$. Теорема доказана.

СЛЕДСТВИЕ 3.8. Если все нули полинома $P(z)=c_{0}+\cdots+z^{n}$ лежат в круге $|z| \leqslant 1$, то существует критическая точка $\zeta$ этого полинома такая, что

$$
|P(\zeta)| \leqslant 1 \text {. }
$$

Равенство достигается для полинома $P(z)=c_{0}+z^{n}$, где $\left|c_{0}\right|=1$. 
Из доказательства теоремы 3.12 видно, что если все нули $z_{\mu}, \mu=1, \ldots, n$, полинома $P(z)=c_{0}+\cdots+z^{n}$ лежат в круге $|z| \leqslant 1$, то справедливо неравенство

$$
\sqrt[n]{\prod_{\mu=1}^{n}\left|P^{\prime}\left(z_{\mu}\right)\right|} \leqslant n
$$

с равенством в случае $P(z)=c_{0}+z^{n},\left|c_{0}\right|=1$. В частности, существует такой нуль $z$, что

$$
\left|P^{\prime}(z)\right| \leqslant n .
$$

Последнее утверждение, а также следствие 3.8 были предложены автору Благовестом Сендовым как ослабленные варианты известных гипотез.

Следующая теорема присутствует в скрытой форме в работе [116; с. 444], где обсуждается ее частный случай (3.6), а доказательство приводится в несколько усложненном виде (ср. также [3; с. 218-219]).

Теорема 3.13. Пусть $\zeta_{\nu}, \nu=1, \ldots, n-1,-$ критические точки полинома $P(z)=c_{1} z+\cdots+z^{n}$, и пусть все нули этого полинома лежат в круге $|z| \leqslant 1$. Тогда

$$
\sqrt[n-1]{\prod_{\nu=1}^{n-1}\left|P\left(\zeta_{\nu}\right)\right|} \leqslant(n-1)\left|\frac{c_{1}^{2}}{n^{n}}\right|^{1 /(n-1)} .
$$

Равенство достигается для полинома $P(z)=c_{1} z+z^{n}$, где $\left|c_{1}\right|=1$.

ДокАЗАТЕльство. Обозначим через $z_{\mu}, \mu=1, \ldots, n$, нули полинома $P$, и пусть $z_{n}=0$. Равенство (3.22) дает

$$
\prod_{\nu=1}^{n-1}\left|P\left(\zeta_{\nu}\right)\right|=n^{-n} \prod_{\substack{\mu, \nu=1 \\ \mu \neq \nu}}^{n}\left|z_{\nu}-z_{\mu}\right|=n^{-n}\left(\prod_{\mu=1}^{n-1}\left|z_{\mu}\right|^{2}\right) \prod_{\substack{\mu, \nu=1 \\ \mu \neq \nu}}^{n-1}\left|z_{\nu}-z_{\mu}\right| .
$$

Как и при доказательстве теоремы 3.12 ,

$$
\prod_{\substack{\mu, \nu=1 \\ \mu \neq \nu}}^{n-1}\left|z_{\nu}-z_{\mu}\right| \leqslant(n-1)^{n-1}
$$

Осталось заметить, что $\prod_{\mu=1}^{n-1}\left|z_{\mu}\right|=\left|c_{1}\right|$. Случай равенства проверяется прямым вычислением. Теорема доказана.

В работе [117] в связи с изучением сходимости метода Ньютона вычисления корней полиномов С. Смейл установил неравенства для критических значений и поставил вопрос о точности этих оценок (см. [117; с. 9, 11, 31-33]). В частности, для полиномов $P(z)=c_{1} z+\cdots+c_{n} z^{n}, c_{1} \neq 0$, он доказал существование критической точки $\zeta$, удовлетворяющей неравенству

$$
|P(\zeta)|\left|\frac{c_{n}}{c_{1}^{n}}\right|^{1 /(n-1)} \leqslant\left(2^{n} \frac{1 \cdot 3 \cdots(2 n-1)}{1 \cdot 2 \cdots(n+1)}\right)^{1 /(n-1)} \leqslant 4
$$

(см. [117; доказательство теоремы 1, с. 9-11]). В статье [13] с помощью диссимметризации функции, заданной на римановой поверхности, получен следующий результат. 
Теорема 3.14. Если $P(z)=c_{1} z+\cdots+z^{n}$, то справедливо неравенство

$$
\min \left\{|P(\zeta)|: P^{\prime}(\zeta)=0\right\} \leqslant(n-1)\left|\frac{c_{1}}{n}\right|^{n /(n-1)} .
$$

Равенство в (3.24) достигается для полиномов вида $P(z)=c_{1} z+z^{n}$, где $c_{1}-$ произвольное комплексное число.

Таким образом, оценка (3.23) улучшается с заменой правых частей на $(n-$ $1)(1 / n)^{n /(n-1)}$, и новая оценка точная. Заметим, что левая часть неравенства (3.24) неоднократно встречается в работе [117].

СлЕДСТВИЕ 3.9. Если все критические точки полинома $P(z)=c_{1} z+\cdots+z^{n}$ лежат в круге $|z| \leqslant 1$, то существует критическая точка $\zeta$ такая, что

$$
|P(\zeta)| \leqslant n-1 \text {. }
$$

Равенство достигается в случае $P(z)=n z+z^{n}$.

Пусть теперь $P$ - произвольный полином со старшим коэффициентом $c_{n}$, и пусть $z-$ произвольное комплексное число. Тогда полином

$$
Q:=\frac{1}{c_{n}}(P(\cdot+z)-P(z))
$$

удовлетворяет условиям теоремы 3.14. Отсюда следует существование критической точки $\zeta\left(\right.$ т. е. $\left.P^{\prime}(\zeta)=0\right)$ такой, что

$$
|P(\zeta)-P(z)| \leqslant(n-1)\left|c_{n}\right|^{1 /(1-n)}\left|\frac{P^{\prime}(z)}{n}\right|^{n /(n-1)} .
$$

Далее, обозначим через $z_{\mu}, \mu=1, \ldots, n$, все нули полинома $P$, и пусть $\zeta_{\nu}$, $\nu=1, \ldots, n-1,-$ нули его производной (пронумерованные с учетом кратности). Полагая в $(3.25) z=z_{j}, 1 \leqslant j \leqslant n$, получаем следующее неравенство между средними геометрическими:

$$
\sqrt[n]{\prod_{\mu=1}^{n}\left|\zeta-z_{\mu}\right|} \leqslant \sqrt[n]{n-1} \sqrt[n-1]{\prod_{\nu=1}^{n-1}\left|z_{j}-\zeta_{\nu}\right|},
$$

справедливое для некоторой критической точки $\zeta$ и любых корней $z_{j}, j=$ $1, \ldots, n$. В частности, если один из корней (пусть $z_{n}$ ) равен нулю, то имеем точное неравенство

$$
\sqrt[n]{\prod_{\mu=1}^{n}\left|\zeta-z_{\mu}\right|} \leqslant \frac{(n-1)^{1 / n}}{n^{1 /(n-1)}} \sqrt[n-1]{\prod_{\mu=1}^{n-1}\left|z_{\mu}\right|} .
$$

Равенство достигается для полинома $P(z)=c_{1} z+z^{n}$. Возвращаясь к прежней нормировке полинома $P(z)=c_{1} z+\cdots+z^{n}$, сравним неравенство $(3.24)$ с гипотезой С. Смейла "о среднем значении" [117; с. 33]. Неравенство (3.24) дает

$$
\min _{1 \leqslant \nu \leqslant n-1}\left|P\left(\zeta_{\nu}\right)\right| \leqslant\left|c_{1}\right| \frac{n-1}{n} \sqrt[n-1]{\prod_{\nu=1}^{n-1}\left|\zeta_{\nu}\right|}
$$


в то время как указанная гипотеза гласит, что

$$
\min _{1 \leqslant \nu \leqslant n-1}\left|\frac{P\left(\zeta_{\nu}\right)}{\zeta_{\nu}}\right| \leqslant\left|c_{1}\right| \frac{n-1}{n}
$$

с равенством для того же полинома $P(z)=c_{1} z+z^{n}, c_{1} \neq 0$. Выписанные неравенства суть близкие, но независимые соотношения. Предположение (3.26) доказано различными методами в ряде частных случаев (см. [3; гл. 7], [116]).

Для произвольного невырожденного континуума $\gamma$ открытой $w$-плоскости обозначим через $F$ функцию Римана:

$$
F(w)=(d(\gamma))^{-1} w+a_{0}+\frac{a_{1}}{w}+\cdots
$$

- конформно и однолистно отображающую связную компоненту множества $\overline{\mathbb{C}}_{w} \backslash \gamma$, содержащую бесконечность, на область $|\zeta|>1$.

ТЕОРема 3.15 [19]. Предположим, что все критические значения полинома $P$ степени $n \geqslant 2$ принадлежат некоторому невырожденному континууму $\gamma \subset \mathbb{C}_{w}$, и пусть точка z такова, что ее образ $P(z)$ принадлежит связной компоненте множества $\overline{\mathbb{C}}_{w} \backslash \gamma$, содержащей бесконечно удаленную точку. Тогда выполняются неравенства

$$
\begin{gathered}
|\zeta-z|\left|P^{\prime}(z)\right| \leqslant \frac{n|F(P(z))|\left(|F(P(z))|^{1 / n}+1\right)}{\left|F^{\prime}(P(z))\right|\left(|F(P(z))|^{1 / n}-1\right)}, \\
|\zeta-z| \geqslant\left(\frac{d(\gamma)}{\left|c_{n}\right|}\right)^{1 / n}\left(|F(P(z))|^{1 /(2 n)}-|F(P(z))|^{-1 /(2 n)}\right)^{2} .
\end{gathered}
$$

Если, дополнительно, $P(1)$ не принадлежит указанной выше связной компоненте, то справедливы такюе неравенства

$$
\frac{|F(P(z))|^{1 / n}-1}{|F(P(z))|^{1 / n}+1} \leqslant\left|\frac{n F(P(z))}{(z-1) P^{\prime}(z) F^{\prime}(P(z))}\right| \leqslant \frac{|F(P(z))|^{1 / n}+1}{|F(P(z))|^{1 / n}-1} .
$$

Здесъ $\zeta$ - произвольная критическая точка полинома $P, c_{n}-$ старший коэффициент этого полинома, $F$ - функиия Римана, соответствующая континууму $\gamma$, a $d(\gamma)$ - постоянная Чебышёва.

ДокАзАтельство. Обозначим через $G$ связную компоненту множества $\overline{\mathbb{C}}_{w} \backslash$ $\gamma$, содержащую бесконечно удаленную точку, и пусть $B=\{z: P(z) \in G\}$. Согласно лемме 2.7 , функция $\Psi(z):=(F(P(z)))^{1 / n}$ распадается в односвязной области $B$ на $n$ регулярных ветвей, каждая из которых однолистно отображает эту область на область $|\zeta|>1$. Далее $\Psi$ означает одну из этих ветвей.

Начнем с доказательства неравенства (3.27). Пусть $z_{0}$ - произвольная фиксированная точка области $B$, а $\zeta$ - произвольная критическая точка полинома $P$, $\zeta \notin B$. В плоскости $\overline{\mathbb{C}}_{z}$ рассмотрим конденсатор $C\left(r ; \mathbb{C}_{z} \backslash \Gamma, \Gamma,\left\{z_{0}\right\},\{0,1\},\{r\}\right)$, где $\Gamma=\left\{z: z=z_{0}+\left(\zeta-z_{0}\right) t, t \geqslant 1\right\}$. Обозначим через $\Gamma^{\prime}$ связное подмножество $\Gamma \cap B$, соединяющее точку $z=\infty$ с границей $\partial B$, а через $\mathrm{Cr}-$ круговую симметризацию относительно вещественной отрицательной полуоси. Из монотонности емкости, конформной инвариантности емкости и принципа круговой симметризации Полиа последовательно получаем

$$
\operatorname{cap} C\left(r ; \mathbb{C}_{z} \backslash \Gamma, \Gamma,\left\{z_{0}\right\},\{0,1\},\{r\}\right) \geqslant \operatorname{cap} C\left(r ; B \backslash \Gamma^{\prime}, \overline{\Gamma^{\prime}},\left\{z_{0}\right\},\{0,1\},\{r\}\right)
$$




$$
\begin{gathered}
=\operatorname{cap} C\left(r ;\left\{\zeta:|\zeta|>1, \zeta \notin \Psi\left(\Gamma^{\prime}\right)\right\}, \Psi\left(\overline{\Gamma^{\prime}}\right),\left\{\Psi\left(z_{0}\right)\right\},\{0,1\},\left\{\left|\Psi^{\prime}\left(z_{0}\right)\right| r\right\}\right) \\
\geqslant \operatorname{cap} \operatorname{Cr} C\left(r ;\left\{\zeta:|\zeta|>1, \zeta \notin \Psi\left(\Gamma^{\prime}\right)\right\}, \Psi\left(\overline{\Gamma^{\prime}}\right),\left\{\Psi\left(z_{0}\right)\right\},\{0,1\},\left\{\left|\Psi^{\prime}\left(z_{0}\right)\right| r\right\}\right) \\
=\operatorname{cap} C\left(r ;\{\zeta:|\zeta|>1, \zeta \notin[-\infty,-1]\},[-\infty,-1],\left\{\left|\Psi\left(z_{0}\right)\right|\right\},\right. \\
\left.\{0,1\},\left\{\left|\Psi^{\prime}\left(z_{0}\right)\right| r\right\}\right) .
\end{gathered}
$$

Ввиду симметрии функции Жуковского $\omega=(\zeta+1 / \zeta) / 2$, последняя емкость равна емкости конденсатора

$$
\begin{gathered}
C\left(r ; \mathbb{C}_{\omega} \backslash[-\infty,-1],[-\infty,-1],\left\{\frac{\left|\Psi\left(z_{0}\right)\right|+1 /\left|\Psi\left(z_{0}\right)\right|}{2}\right\},\right. \\
\left.\{0,1\},\left\{\frac{\left.|1-| \Psi\left(z_{0}\right)\right|^{-2} \mid}{2}\left|\Psi^{\prime}\left(z_{0}\right)\right| r\right\}\right) .
\end{gathered}
$$

Сравнивая асимптотику емкости этого конденсатора с асимптотикой емкости конденсатора $C\left(r ; \mathbb{C}_{z} \backslash \Gamma, \Gamma,\left\{z_{0}\right\},\{0,1\},\{r\}\right)$ при $r \rightarrow 0$, приходим к неравенству

$$
4\left|\zeta-z_{0}\right| \frac{\left.|1-| \Psi\left(z_{0}\right)\right|^{-2} \mid}{2}\left|\Psi^{\prime}\left(z_{0}\right)\right| \leqslant 4\left(1+\frac{\left|\Psi\left(z_{0}\right)\right|+1 /\left|\Psi\left(z_{0}\right)\right|}{2}\right)
$$

(см. формулу (3.4)). Отсюда следует неравенство (3.27) для $z=z_{0}$.

Для доказательства (3.28) рассмотрим конденсатор $C\left(r ; \overline{\mathbb{C}}_{z} \backslash\left[z_{0}, \zeta\right],\left[z_{0}, \zeta\right]\right.$, $\{\infty\},\{0,1\},\{r\})$, где $z_{0}$ и $\zeta$ определены выше. Вновь из монотонности, конформной инвариантности, принципа круговой симметризации и симметрии функции Жуковского получаем

$$
\begin{aligned}
\operatorname{cap} C & \left(r ; \overline{\mathbb{C}}_{z} \backslash L, L,\{\infty\},\{0,1\},\{r\}\right) \geqslant \operatorname{cap} C\left(r ; B \backslash L^{\prime}, \overline{L^{\prime}},\{\infty\},\{0,1\},\{r\}\right) \\
& =\operatorname{cap} C\left(r ;\left\{\zeta:|\zeta|>1, \zeta \notin \Psi\left(L^{\prime}\right)\right\}, \Psi\left(\overline{L^{\prime}}\right),\{\infty\},\{0,1\},\left\{\left(\frac{d(\gamma)}{\left|c_{n}\right|}\right)^{1 / n} r\right\}\right) \\
& \geqslant \operatorname{cap} C r C\left(r ;\left\{\zeta:|\zeta|>1, \zeta \notin \Psi\left(L^{\prime}\right)\right\}, \Psi\left(\overline{L^{\prime}}\right),\{\infty\},\{0,1\},\left\{\left(\frac{d(\gamma)}{\left|c_{n}\right|}\right)^{1 / n} r\right\}\right) \\
= & \operatorname{cap} C\left(r ;\left\{\zeta:|\zeta|>1, \zeta \notin\left[-\left|\Psi\left(z_{0}\right)\right|,-1\right]\right\},\right. \\
= & \operatorname{cap} C\left(r ; \overline{\mathbb{C}}_{\omega} \backslash\left[-\frac{\left|\Psi\left(z_{0}\right)\right|+1 /\left|\Psi\left(z_{0}\right)\right|}{2},-1\right],\right. \\
& {\left.\left[-\frac{\left|\Psi\left(z_{0}\right)\right|+1 /\left|\Psi\left(z_{0}\right)\right|}{2},-1\right],\{\infty\},\{0,1\},\left\{2\left(\frac{d(\gamma)}{\left|c_{n}\right|}\right)^{1 / n} r\right\}\right) . }
\end{aligned}
$$

Здесь $L=\left[z_{0}, \zeta\right]$, а $L^{\prime}-$ связное подмножество $L \cap B$, соединяющее точку $z_{0}$ с границей $\partial B$.

Сравнение асимптотик первой и последней емкости в выписанных соотношениях при $r \rightarrow 0$ дает неравенство

$$
\left|\zeta-z_{0}\right| \geqslant 2\left(\frac{d(\gamma)}{\left|c_{n}\right|}\right)^{1 / n}\left[\frac{1}{2}\left(\left|\Psi\left(z_{0}\right)\right|+\frac{1}{\left|\Psi\left(z_{0}\right)\right|}\right)-1\right],
$$

которое совпадает с неравенством (3.28). 
Перейдем теперь к доказательству неравенства (3.29). Из свойств функции $\Psi$ и условия $P(1) \notin G$ следует, что функция $z=f(\omega)$, обратная к суперпозиции

$$
\omega=\left(\Psi\left(\left(\frac{d(\gamma)}{c_{n}}\right)^{1 / n} \frac{1}{z}+1\right)\right)^{-1}
$$

принадлежит известному классу $S$. Для таких функций справедлива двусторонняя оценка

$$
\frac{1-|\omega|}{1+|\omega|} \leqslant\left|\frac{\omega f^{\prime}(\omega)}{f(\omega)}\right| \leqslant \frac{1+|\omega|}{1-|\omega|},
$$

$0<|\omega|<1$ (см., например, [85; с. 35]). После простых вычислений убеждаемся, что данная оценка влечет за собой неравенство (3.29). Теорема доказана.

Отметим частные случаи неравенств (3.27) и (3.28), когда все критические значения полинома $P$ принадлежат кругу $\left|w-w_{0}\right| \leqslant R$. В этом случае $\gamma=$ $\left\{w:\left|w-w_{0}\right| \leqslant R\right\}, F(w)=\left(w-w_{0}\right) / R$ и $d(\gamma)=R$. Из неравенства (3.27) вытекает оценка

$$
|\zeta-z|\left|P^{\prime}(z)\right|\left(\left|P(z)-w_{0}\right|-R^{1 / n}\right) \leqslant n\left|P(z)-w_{0}\right|\left(\left|P(z)-w_{0}\right|+R^{1 / n}\right),
$$

справедливая для любой точки $z \in \mathbb{C}$. Полагая $w_{0}=0$, получаем следующее утверждение.

СЛЕДСТвИЕ 3.10. Если $P(z)=1+c_{1} z+\cdots+c_{n} z^{n}, n \geqslant 2, c_{n} \neq 0$, mo существует по крайней мере одна критическая точка $а, P^{\prime}(a)=0$, такая, что

$$
\left|\zeta P^{\prime}(0)\right|\left(1-|P(a)|^{1 / n}\right) \leqslant n\left(1+|P(a)|^{1 / n}\right)
$$

для любых критических точек $\zeta$. Равенство достигается для полинома $P(z)=$ $(1+t z)^{n}$, где $t$ - произвольное комплексное число, отличное от нуля.

Неравенство (3.28) дает следующее утверждение.

СлЕДСтвиЕ 3.11. Если $P(z)=1+c_{1} z+\cdots+c_{n} z^{n}, n \geqslant 2, c_{n} \neq 0$, mo существует по крайней мере одна критическая точка $а, P^{\prime}(a)=0$, такая, что либо $|P(a)| \geqslant 1$, либо

$$
|\zeta| \geqslant\left(\frac{1}{\left|c_{n}\right|}\right)^{1 / n}\left(|P(a)|^{1 / n}-1\right)^{2}
$$

для любых критических точек $\zeta$. Равенство в последнем соотношении выполняется в случае, когда $P(z)=(1+t z)^{n}, t \neq 0$.

Рассмотрим также частный случай неравенства $(3.29)$, когда $\gamma=[\bar{L}(P), \bar{H}(P)]$. Функция $F$ конформно и однолистно отображает внешность отрезка $\gamma$ на область $|\zeta|>1$, и, следовательно, обратная функция имеет вид

$$
F^{-1}(\zeta)=\frac{1}{2}\left(\zeta+\frac{1}{\zeta}\right) \frac{\bar{H}(P)-\bar{L}(P)}{2}+\frac{\bar{H}(P)+\bar{L}(P)}{2}=\frac{1}{4}(\bar{H}(P)-\bar{L}(P)) \zeta+\cdots,
$$

$d(\gamma)=(\bar{H}(P)-\bar{L}(P)) / 4$. Неравенство (3.29) дает следующее утверждение. 
СлеДСтвиЕ 3.12. Для любого полинома $P$ степени $n$ с вещественными коэффициентами и с критическими точками на отрезке $[-1,1]$ выполняются неравенства

$$
\frac{|F(P(z))|^{1 / n}-1}{|F(P(z))|^{1 / n}+1} \leqslant\left|\frac{n(\bar{H}(P)-\bar{L}(P))\left[F^{2}(P(z))-1\right]}{4(z-1) P^{\prime}(z) F(P(z))}\right| \leqslant \frac{|F(P(z))|^{1 / n}+1}{|F(P(z))|^{1 / n}-1},
$$

где z - произвольная точка плоскости $\mathbb{C}$, для которой $P(z) \notin[\bar{L}(P), \bar{H}(P)]$. Равенство в левом неравенстве (3.30) достигается для полинома Чебъшёва $T_{n}(z)=2^{n-1} z^{n}+\cdots$ при $-\infty<z<-1$, а в правом неравенстве (3.30) - для того же полинома и $1<z<\infty$.

Используя только свойства емкостей конденсаторов (без привлечения симметризации), можно получить следующие два утверждения [22].

ТеОрема 3.16. Предположим, что все критические значения полинома $Р$ степени $n \geqslant 2$ принадлежат некоторому невырожденному континууму $\gamma \subset \mathbb{C}_{w}$, и пусть точка z такова, что ее образ $P(z)$ принадлежит связной компоненте $\overline{\mathbb{C}}_{w} \backslash \gamma$, содержащей бесконечность. Тогда выполняется неравенство

$$
\left|P^{\prime}(z)\right| \leqslant n\left|\frac{c_{n}}{d(\gamma)}\right|^{1 / n} \frac{\left|F(P(z)) / F^{\prime}(P(z))\right|}{|F(P(z))|^{1 / n}-|F(P(z))|^{-1 / n}},
$$

где $c_{n}, F u d(\gamma)$ из теореми 3.15 .

Теорема 3.17. Если $P(z)=1+c_{1} z+\cdots+c_{n} z^{n}, n \geqslant 2, c_{n} \neq 0$, mо существует по крайней мере одна критическая точка $а, P^{\prime}(a)=0$, такая, что

$$
|P(a)|^{2 / n} \geqslant 1-n \frac{\left|c_{n}\right|^{1 / n}}{\left|c_{1}\right|} .
$$

Равенство достигается для полинома $P(z)=(1+t z)^{n}$, где $t-$ произвольное комплексное число, отличное от нуля.

\section{Список литературы}

[1] G. V. Milovanović, D. S. Mitrinović, Th. M. Rassias, Topics in polynomials: extremal problems, inequalities, zeros, World Scientific Publishing Co., Inc., River Edge, NJ, 1994, xiv+821 pp.

[2] P. Borwein, T. Erdélyi, Polynomials and polynomial inequalities, Grad. Texts in Math., 161, Springer-Verlag, New York, 1995, x+480 pp.

[3] Q. I. Rahman, G. Schmeisser, "Analytic theory of polynomials", London Math. Soc. Monogr. (N.S.), 26, The Clarendon Press, Oxford; Oxford Univ. Press, 2002, xiv $+742 \mathrm{pp}$.

[4] T. Sheil-Small, Complex polynomials, Cambridge Stud. Adv. Math., 75, Cambridge Univ. Press, Cambridge, 2002, xx+428 pp.

[5] В. В. Прасолов, Многочлены, изд. 3-е, испр., МЦНМО, М., 2003, 336 с.; англ. пер. 2-го изд.: V.V. Prasolov, Polynomials, Algorithms Comput. Math., 11, Springer-Verlag, Berlin, 2004, xiv+301 pp.

[6] А.Б. Богатырёв, Экстремальные многочленъ и римановы поверхности, МЦНМО, М., 2005, 173 с.; англ. пер.: А. Bogatyrev, Extremal polynomials and Riemann surfaces, Springer Monogr. Math., Springer, Berlin, 2012, xxv+150 pp. 
[7] В.Н. Дубинин, "Теоремы искажения для полиномов на окружности”, Матем. сб., 191:12 (2000), 51-60; англ. пер.: V. N. Dubinin, "Distortion theorems for polynomials on a circle", Sb. Math., 191:12 (2000), 1797-1807.

[8] В.Н. Дубинин, "Конформные отображения и неравенства для алгебраических полиномов", Алгебра и анализ, 13:5 (2001), 16-43; англ. пер.: V. N. Dubinin, "Conformal mappings and inequalities for algebraic polynomials", St. Petersburg Math. J., 13:5 (2002), 717-737.

[9] В.Н. Дубинин, "О применении конформных отображений в неравенствах для рациональных функций”, Изв. РАН. Сер. матем., 66:2 (2002), 67-80; англ. пер.: V.N. Dubinin, "On an application of conformal maps to inequalities for rational functions", Izv. Math., 66:2 (2002), 285-297.

[10] В.Н. Дубинин, "Конформные отображения и неравенства для алгебраических полиномов. II", Аналитическая теория чисел и теория функиий. 19, Зап. научн. сем. ПОМИ, 302, ПОМИ, СПб., 2003, 18-37; англ. пер.: V. N. Dubinin, "Conformal mappings and inequalities for algebraic polynomials. II", J. Math. Sci. (N. Y.), 129:3 (2005), 3823-3834.

[11] В. Н. Дубинин, “Лемма Шварца и оценки коэффициентов для регулярных функций со свободной областью определения", Матем. сб., 196:11 (2005), 53-74; англ. пер.: V. N. Dubinin, "Schwarz's lemma and estimates of coefficients for regular functions with free domain of definition", Sb. Math., 196:11 (2005), 1605-1625.

[12] В.Н. Дубинин, "О полиномах с критическими значениями на отрезке", Maтем. заметки, 78:6 (2005), 827-832; англ. пер.: V. N. Dubinin, "Polynomials with critical values on intervals", Math. Notes, 78:6 (2005), 768-772.

[13] В. Н. Дубинин, "Неравенства для критических значений полиномов", Матем. сб., 197:8 (2006), 63-72; англ. пер.: V. N. Dubinin, "Inequalities for critical values of polynomials", Sb. Math., 197:8 (2006), 1167-1176.

[14] В.Н.Дубинин, "Лемниската и неравенства для логарифмической емкости континуума", Матем. заметки, 80:1 (2006), 33-37; англ. пер.: V.N. Dubinin, "Lemniscates and inequalities for the logarithmic capacities of continua", Math. Notes, 80:1-2 (2006), 31-35.

[15] В.Н.Дубинин, "О применении леммы Шварца к неравенствам для целых функций с ограничениями на нули", Аналитическая теория чисел и теория функиий. 21, Зап. научн. сем. ПОМИ, 337, ПОМИ, СПб., 2006, 101-112; англ. пер.: V.N. Dubinin, "Applications of the Schwarz lemma to inequalities for entire functions with constraints on zeros", J. Math. Sci. (N. Y.), 143:3 (2007), 3069-3076.

[16] В. Н. Дубинин, "О принципах мажорации для мероморфных функций”, Матем. заметки, 84:6 (2008), 803-808; англ. пер.: V. N. Dubinin, "Majorization principles for meromorphic functions", Math. Notes, 84:5-6 (2008), 751-755.

[17] В.Н. Дубинин, "О компонентах лемнискаты, не содержащих критических точек полинома, отличных от его нулей", Аналитическая теория чисел и теория функиий. 25, Зап. научн. сем. ПОМИ, 383, ПОМИ, СПб., 2010, 77-85; англ. пер.: V. N. Dubinin, "On the lemniscate components containing no critical points of a polynomial except for its zeros", J. Math. Sci. (N. Y.), 178:2 (2011), 158-162.

[18] В.Н. Дубинин, "K теореме о конечном приращении для комплексных полиномов", Матем. заметки, 88:5 (2010), 673-682; англ. пер.: V. N. Dubinin, "On the finite-increment theorem for complex polynomials", Math. Notes, 88:5-6 (2010), 647-654.

[19] В. Н. Дубинин, "К теоремам искажения для алгебраических полиномов", Далъневост. матем. журн., 11:1 (2011), 28-36.

[20] В.Н.Дубинин, "Нижняя граница для дискретной нормы полинома на окружности", Матем. заметки, 90:2 (2011), 306-309; англ. пер.: V. N. Dubinin, "Lower 
bound for the discrete norm of a polynomial on the circle", Math. Notes, 90:1-2 (2011), 284-287.

[21] В.Н. Дубинин, "Новая версия круговой симметризации с приложениями к р-листным функциям", Матем. сб., 203:7 (2012), 79-94.

[22] В.Н.Дубинин, В.Ю.Ким, "Приведенные модули и неравенства для полиномов", Аналитическая теория чисел и теория функиий. 16, Зап. научн. сем. ПОМИ, 263, ПОМИ, СПб., 2000, 70-83; англ. пер.: V. N. Dubinin, "Reduced modules and inequalities for polynomials", J. Math. Sci. (N. Y.), 110:6 (2002), 3070-3077.

[23] В.Н. Дубинин, А.В. Олесов, "О применении конформных отображений к неравенствам для полиномов", Аналитическая теория чисел и теория функuий. 18, Зап. научн. сем. ПОМИ, 286, ПОМИ, СПб., 2002, 85-102; англ. пер.: V. N. Dubinin, A. V. Olesov, "Application of conformal mappings to inequalities for polynomials", J. Math. Sci. (N. Y.), 122:6 (2004), 3630-3640.

[24] В.Н.Дубинин, С. И. Калмыков, "Экстремальные свойства полиномов Чебышёва", Дальневост. матем. журн., 5:2 (2004), 169-177.

[25] В. Н. Дубинин, С. И. Калмыков, "Принцип мажорации для мероморфных функций", Матем. сб., 198:12 (2007), 37-46; англ. пер.: V. N. Dubinin, S. I. Kalmykov, "A majoration principle for meromorphic functions", Sb. Math., 198:12 (2007), 1737-1745.

[26] V. Dubinin, T. Sugawa, "Dual mean value problem for complex polynomials", Proc. Japan. Acad. Ser. A Math. Sci., 85:9 (2009), 135-137.

[27] В.Н.Дубинин, Д. А. Кириллова, "Некоторые применения экстремальных разбиений в геометрической теории функций”, Далъневост. матем. журн., 10:2 (2010), 130-152.

[28] В.Н.Дубинин, С. И. Калмыков, "О полиномах с ограничениями на дугах окружностей", Аналитическая теория чисел и теория функиий. 26, Зап. научн. сем. ПОМИ, 392, ПОМИ, СПб., 2011, 74-83.

[29] С. И. Калмыков, “Теоремы покрытия для полиномов, имеющих криволинейную мажоранту на двух отрезках", Аналитическая теория чисел и теория функций. 25, Зап. научн. сем. ПОМИ, 383, ПОМИ, СПб., 2010, 97-109; англ. пер.: S. I. Kalmykov, "Covering theorems for polynomials with curved majorants on two intervals", J. Math. Sci. (N. Y.), 178:2 (2011), 170-177.

[30] С. И. Калмыков, "О полиномах, имеющих криволинейную мажоранту на двух отрезках", Изв. вузов. Матем., 2009, № 10, 72-75; англ. пер.: S. I. Kalmykov, "Polynomials with curved majorants on two segments", Russian Math. (Iz. VUZ), 53:10 (2009), 64-67.

[31] С. И. Калмыков, "Об оценке модуля рациональной функции", Аналитическая теория чисел и теория функиий. 24, Зап. научн. сем. ПОМИ, 371, ПОМИ, СПб., 2009, 109-116; англ. пер.: S. I. Kalmykov, "An estimate for the modulus of a rational function", J. Math. Sci. (N. Y.), 166:2 (2010), 186-190.

[32] С. И. Калмыков, "Принципы мажорации и некоторые неравенства для полиномов и рациональных функций с предписанными полюсами", Зап. научн. сем. ПОМИ, 357, ПОМИ, СПб., 2008, 143-157; англ. пер.: S. I. Kalmykov, "Majoration principles and some inequalities for polynomials and rational functions with prescribed poles", J. Math. Sci. (N. Y.), 157:4 (2009), 623-631.

[33] А.В. Олесов, “Дифференциальные неравенства для алгебраических полиномов", Сиб. матем. журн., 51:4 (2010), 883-889; англ. пер.: A. V. Olesov, "Differential inequalities for algebraic polynomials", Siberian Math. J., 51:4 (2010), 706-711.

[34] А.В. Олесов, "О применении конформных отображений к неравенствам для тригонометрических полиномов", Матем. заметки, 76:3 (2004), 396-408; англ. 
пер.: A.V. Olesov, "Application of conformal mappings to inequalities for trigonometric polynomials", Math. Notes, 76:3-4 (2004), 368-378.

[35] А. В. Олесов, "Неравенства для целых функций конечной степени и полиномов", Аналитическая теория чисел и теория функиий. 20, Зап. научн. сем. ПОМИ, 314, ПОМИ, СПб., 2004, 174-195; англ. пер.: А. V. Olesov, "Inequalities for entire functions of finite degree and polynomials", J. Math. Sci. (N. Y.), 133:6 (2006), 1704-1717.

[36] А.В. Олесов, "Неравенства для мажорантных аналитических функций”, Аналитическая теория чисел и теория функиий. 20, Зап. научн. сем. ПОМИ, 314, ПОМИ, СПб., 2004, 155-173; англ. пер.: A. V. Olesov, "Inequalities for majorizing analytic functions", J. Math. Sci. (N. Y.), 133:6 (2006), 1693-1703.

[37] В.Н. Дубинин, "Симметризация в геометрической теории функций комплексного переменного", УМH, 49:1(295) (1994), 3-76; англ. пер.: V.N. Dubinin, "Symmetrization in the geometric theory of functions of a complex variable", Russian Math. Surveys, 49:1 (1994), 1-79.

[38] В.Н. Дубинин, Емкости конденсаторов и симметризация в геометрической теории функиий комплексного переменного, Дальнаука, Владивосток, 2009, $390 \mathrm{c}$.

[39] Г. М. Голузин, Геометрическая теория функиий комплексного переменного, 2-е изд., Наука, М., 1966, 628 с.

[40] К. Дочев, "О некоторых экстремальных свойствах многочленов", Докл. АН CCCP, 153 (1963), 519-521; англ. пер.: K. Dochev, "Some extremal properties of polynomials", Soviet Math. Dokl., 4 (1963), 1704-1706.

[41] R. Duffin, A. C. Schaeffer, "Some properties of functions of exponential type", Bull. Amer. Math. Soc., 44:4 (1938), 236-240.

[42] T. Sheil-Small, "An inequality for the modulus of a polynomial evaluated at the roots of unity", Bull. Lond. Math. Soc., 40:6 (2008), 956-964.

[43] E. Rakhmanov, B. Shekhtman, "On discrete norms of polynomials", J. Approx. Theory, 139:1-2 (2006), 2-7.

[44] P. Borwein, T. Erdélyi, J. Zhang, "Chebyshev polynomials and Markov-Bernstein type inequalities for rational spaces", J. London Math. Soc. (2), 50:3 (1994), 501-519.

[45] P. Borwein, T. Erdélyi, "Sharp extensions of Bernstein's inequality to rational spaces", Mathematika, 43:2 (1996), 413-423.

[46] X. Li, R. N. Mohapatra, R. S. Rodriguez, "Bernstein-type inequalities for rational functions with prescribed poles", J. London Math. Soc. (2), 51:3 (1995), 523-531.

[47] R. Jones, X. Li, R. N. Mohapatra, R. S. Rodrigues, "On the Bernstein inequality for rational functions with a prescribed zero", J. Approx. Theory, 95:3 (1998), 476-496.

[48] X. Li, "Integral formulas and inequalities for rational functions", J. Math. Anal. Appl., 211:2 (1997), 386-394.

[49] A. Aziz, W. M. Shah, "Some refinements of Bernstein-type inequalities for rational functions", Glas. Mat. Ser. III, 32(52):1 (1997), 29-37.

[50] G. Min, "Inequalities for rational functions with prescribed poles", Canad. J. Math., 50:1 (1998), 152-166.

[51] В.Н. Русак, Рационалъные функиии как аппарат приближения, Изд-во БГУ, Минск, 1979, 174 с.

[52] В. С. Виденский, "Некоторые оценки производных от рациональных дробей", Изв. РАН СССР. Сер. матем., 26:3 (1962), 415-426.

[53] R. Osserman, "A sharp Schwarz inequality on the boundary", Proc. Amer. Math. Soc., 128:12 (2000), 3513-3517. 
[54] В.Н. Дубинин, "K неравенству Шварца на границе для регулярных в круге функций", Аналитическая теория чисел и теория функций. 18, Зап. научн. сем. ПОМИ, 286, ПОМИ, СПб., 2002, 74-84; англ. пер.: V. N. Dubinin, "The Schwarz inequality on the boundary for functions regular in the disk", J. Math. Sci. (N. Y.), 122:6 (2004), 3623-3629.

[55] А. Ю. Солынин, "Граничное искажение и экстремальные задачи в некоторых классах однолистных функций", Аналитическая теория чисел и теория функиий. 11, Зап. научн. сем. ПОМИ, 204, ПОМИ, СПб., 1993, 115-142; англ. пер.: A. Yu. Solynin, "The boundary distortion and extremal problems in certain classes of univalent functions", J. Math. Sci. (N. Y.), 79:5 (1996), 1341-1358.

[56] H. P. Boas, "Julius and Julia: mastering the art of the Schwarz lemma", Amer. Math. Monthly, 117:9 (2010), 770-785.

[57] S. G. Krantz, "The Schwarz lemma at the boundary", Complex Var. Elliptic Equ., 56:5 (2011), 455-468.

[58] A. Aziz, Q. G. Mohammad, "Growth of polynomials with zeros outside a circle", Proc. Amer. Math. Soc., 81:4 (1981), 549-553.

[59] N. C. Ankeny, T. J. Rivlin, "On a theorem of S. Bernstein", Pacific J. Math., 5, Suppl. 2 (1955), 849-852.

[60] P. Turan, "Über die Ableitung von Polynomen", Compositio Math., 7 (1939), 89-95.

[61] P. Pawlowski, "On the zeros of a polynomial and its derivatives", Trans. Amer. Math. Soc., 350:11 (1998), 4461-4472.

[62] С.Н. Бернштейн, Собрание сочинений, т. 2: Конструктивная теория функиий (1931-1953), Из-во АН СССР, М., 1954, 627 с.

[63] B. Ya. Levin, Lectures on entire functions, Transl. Math. Monog., 150, Amer. Math. Soc., Providence, RI, 1996, xvi+248 pp.

[64] R. Gardner, N.K. Govil, "Functions of exponential type not vanishing in a half-plane", Analysis, 17:4 (1997), 395-402.

[65] N. K. Govil, M. A. Qazi, "On maximum modulus of polynomials and related entire functions with restricted zeros", Math. Inequal. Appl., 5:1 (2002), 57-60.

[66] N. K. Govil, M. A. Qazi, Q. I. Rahman, "A new property of entire functions of exponential type not vanishing in a half-plane and applications", Complex Var. Theory Appl., 48:11 (2003), 897-908.

[67] Т. Г. Генчев, "Неравенства для асимметрических целых функций экспоненциального типа", Докл. АН ССCP, 241:6 (1978), 1261-1264; англ. пер.: Т. G. Genchev, "Inequalities for asymmetric entire functions of exponential type", Soviet Math. Dokl., 19 (1978), 981-985.

[68] С. Стоилов, Теория функиий комплексного переменного, т. 2, ИЛ, М., 1962, 413 с.; пер. с рум.: S. Stoilow, Teoria funcţiilor de o variabilă complexă, v. 2: Funcţii armonice. Suprafeţe Riemanniene, Editura Academiei Republicii Populare Romîne, Bucharest, 1958, 378 pp.

[69] V. N. Dubinin, M. Vuorinen, "Robin functions and distortion theorems for regular mappings", Math. Nachr., 283:11 (2010), 1589-1602.

[70] И. П. Митюк, "Принцип симметризации для многосвязных областей”, Докл. АН CCCP, 157 (1964), 268-270; англ. пер.: I. P. Mityuk, "The symmetrization principle for multiply connected domains", Soviet Math. Dokl., 5 (1964), 928-930.

[71] И.П. Митюк, "Принцип симметризации для многосвязных областей и некоторые его применения", Укр. матем. журн., 17:4 (1965), 46-54.

[72] И. П. Митюк, Симметризачионные методы и их применение в геометрической теории функиий. Введение в симметризационные методы, Изд-во Кубанск. гос. ун-та, Краснодар, 1980, 90 с.

[73] И.П. Митюк, Применение симметризационных методов в геометрической теории функций, Изд-во Кубанск. гос. ун-та, Краснодар, 1985, 94 с. 
[74] И. П. Митюк, “Оценки внутреннего радиуса (емкости) некоторой области (конденсатора)", Изв. Северо-Кавказ. научн. иентра высш. шк. естеств. наук, 43:3 (1983), 36-38.

[75] А.Л. Лукашов, "Неравенства для производных рациональных функций на нескольких отрезках", Изв. РАН. Сер. матем., 68:3 (2004), 115-138; англ. пер.: A. L. Lukashov, "Inequalities for the derivatives of rational functions on several intervals", Izv. Math., 68:3 (2004), 543-565.

[76] А.Л. Лукашов, “Оценки производных рациональных функций и четвертая задача Золотарева", Алгебра и анализ, 19:2 (2007), 122-130; англ. пер.: A.L. Lukashov, "Estimates for derivatives of rational functions and the fourth Zolotarev problem", St. Petersburg Math. J., 19:2 (2008), 253-259.

[77] С. В. Тышкевич, "О чебышевских полиномах на дугах окружности", Матем. заметки, 81:6 (2007), 952-954; англ. пер.: S. V. Tyshkevich, "On Chebyshev polynomials on arcs of a circle", Math. Notes, 81:6 (2007), 851-853.

[78] Л. С. Маергойз, Н. Н. Рыбакова, "Многочлены Чебышёва с нулевым множеством на дуге окружности”, Докл. РАН, 426:1 (2009), 26-28; англ. пер.: L. S. Maergoŭz, N. N. Rybakova, "Chebyshev polynomials with zeros lying on a circular arc", Dokl. Math., 79:3 (2009), 319-321.

[79] А. Л. Лукашов, С. В. Тышкевич, “Экстремальные полиномы на дугах окружности с нулями на этих дугах", Изв. НАН Армении. Матем., 44:3 (2009), 41-50; англ. пер.: A.L. Lukashov, S. V. Tyshkevich, "Extremal polynomials on arcs of the circle with zeros on these arcs", J. Contemp. Math. Anal., 44:3 (2009), 172-179.

[80] В.В. Арестов, А.С. Менделев, "О тригонометрических полиномах, наименее уклоняющихся от нуля", Докл. РАН, 425:6 (2009), 733-736; англ. пер.: V. V. Arestov, A. S. Mendelev, "On trigonometric polynomials least deviating from zero", Dokl. Math., 79:2 (2009), 280-283.

[81] В. С. Виденский, "Экстремальные оценки производной тригонометрического полинома на отрезке, меньшем чем период", Докл. АН СССР, 130 (1960), 13-16; англ. пер.: V.S. Videnskii, "Extremal estimates for the derivative of a trigonometric polynomial on an interval shorter than its period", Soviet Math. Dokl., 1 (1960), 5-8.

[82] J.-P. Thiran, C. Detaille, "Chebyshev polynomials on circular arcs in the complex plane", Progress in approximation theory, Academic Press, Boston, MA, 1991, $771-786$.

[83] Дж. Дженкинс, Однолистные функции и конформные отображения, ИЛ, М., 1962, 266 с.; пер. с англ.: J. A. Jenkins, Univalent functions and conformal mapping, Ergeb. Math. Grenzgeb. Neue Folge, 18, Reihe: Moderne Funktionentheorie, Springer-Verlag, Berlin-Gottingen-Heidelberg, 1958, vi+169 pp.

[84] A. W. Goodman, Univalent functions, v. I, II, Tampa, FL, Mariner Publishing Co., Inc., 1983, xvii+246; xii+311 pp.

[85] P. L. Duren, Univalent functions, Grundlehren Math. Wiss., 259, Springer-Verlag, New York, 1983, xiv+382 pp.

[86] Ch. Pommerenke, Boundary behaviour of conformal maps, Grundlehren Math. Wiss., 299, Springer-Verlag, Berlin, 1992, x+300 pp.

[87] G. Szegő, "Über einen Satz des Herrn Serge Bernstein", Schriften Königsberg. Gel. Ges., 5 (1928), 59-70.

[88] V.K. Jain, "Generalization of certain well known inequalities for polynomials", Glas. Mat. Ser. III, 32(52):1 (1997), 45-51.

[89] A. Aziz, W. M. Shah, "Inequalities for the polar derivative of a polynomial", Indian J. Pure Appl. Math., 29:2 (1998), 163-173.

[90] P. J. O'Hara, R. S. Rodriguez, "Some properties of self-inversive polynomials", Proc. Amer. Math. Soc., 44:2 (1974), 331-335. 
[91] A. Aziz, B. A. Zargar, "Some inequalities for self-reciprocal polynomials", Indian J. Pure Appl. Math., 27:8 (1996), 791-794.

[92] V.K. Jain, "Inequalities for polynomials satisfying $p(z) \equiv z^{n} p(1 / z)$. III", J. Indian. Math. Soc. (N.S.), 62:1-4 (1996), 1-4.

[93] M. A. Malik, "On the derivative of polynomial", J. London Math. Soc. (2), 1 (1969), $57-60$.

[94] Г. Полиа, Г. Сеге, Задачи и теоремъ из анализа, т. І: Ряды. Интегральное исчисление. Теория функиий, 3-е рус. изд., Наука, М., 1978, 392 с.; пер. с нем.: G. Pólya, G. Szegő, Aufgaben und Lehrsätze aus der Analysis, v. I: Reihen, Integralrechnung, Funktionentheorie, Dritte berichtigte Auflage, Grundlehren Math. Wiss., 19, Springer-Verlag, Berlin-New York, 1964, xvi+338 pp.

[95] В. И. Смирнов, "Sur quelques polynômes aux propriétés extrémales", Заn. Харък. Матем. общ-ва, 4:2 (1928), 69-72.

[96] M. A. Malik, M. C. Vong, "Inequalities concerning the derivative of polynomials", Rend. Circ. Mat. Palermo (2), 34:3 (1985), 422-426.

[97] В. И. Смирнов, Н. А. Лебедев, Конструктивная теория функиий комплексного переменного, Наука, М.-Л., 1964, 438 с.

[98] Н. А. Лебедев, "Некоторые оценки для функций, регулярных и однолистных в круге", Вестн. Ленингр. ун-та. Сер. матем., физ., хим., 10:11 (1955), 3-21.

[99] С.Н. Бернштейн, Собрание сочинений, т. 1: Конструктивная теория функиий (1905-1930), Изд-во АН СССР, М., 1952, 581 с.

[100] P. Turan, "Über die Ableitung von Polynomen", Compositio Math., 7 (1939), 89-95.

[101] Z. Nehari, "Some inequalities in the theory of functions", Trans. Amer. Math. Soc., 75:2 (1953), 256-286.

[102] Н. А. Лебедев, Принцип площадей в теории однолистных функиий, Наука, М., 1975, $336 \mathrm{c}$.

[103] D. V. Prokhorov, Reachable set methods in extremal problems for univalent functions, Saratov Univ. Publ. House, Saratov, 1993, 228 pp.

[104] В.А. Марковъ, О функиілхг, наименпе уклоняющихся отг нуля вг данномг промежуткю, СПб., 1892, 117 с.

[105] J. Erőd, "Bizonyos polinomok mazimumának", Mat. Fiz. Lapok, 46 (1939), 58-83.

[106] H. Siejka, O. Tammi, "On estimating the inverse coefficients for meromorphic univalent functions omitting a disk", Ann. Acad. Sci. Fenn. Ser. A I Math., 12:1 (1987), 85-93.

[107] Ch. Pommerenke, A. Vasil'ev, "Angular derivatives of bounded univalent functions and extremal partitions of the unit disk", Pacific J. Math., 206:2 (2002), 425-450.

[108] Я.Л. Геронимус, Теория ортогональных многочленов, ГИТТЛ, М.-Л., 1950, $164 \mathrm{c}$.

[109] В.И. Лебедев, Функииональный анализ и вычислительная математика, изд. 4-е, испр., Физматлит, М., 2000, 295 с.

[110] M. A. Lachance, "Bernstein and Markov inequalities for constrained polynomials", Rational approximation and interpolation (Tampa, FL, 1983), Lecture Notes in Math., 1105, Springer, Berlin, 1984, 125-135.

[111] Q. I. Rahman, "On a problem of Turán about polynomials with curved majorants", Trans. Amer. Math. Soc., 163 (1972), 447-455.

[112] Г. Полиа, Г. Сеге, Изопериметрические неравенства в математической физике, Физматгиз, М., 1962, 336 с.; пер. с англ.: G. Pólya, G. Szegö, Isoperimetric Inequalties in Mathematical Physics, Annals of Mathematics Studies, 27, Princeton Univ. Press, Princeton, NJ, 1951.

[113] W.K. Hayman, Multivalent functions, 2nd ed., Cambridge Tracts in Math., 110, Cambridge Univ. Press, Cambridge, 1994, xii+263 pp. 
[114] В.Н.Дубинин, "Асимптотика модуля вырождающегося конденсатора и некоторые ее применения", Аналитическая теория чисел и теория функиий. 14, Зап. научн. сем. ПОМИ, 237, ПОМИ, СПб., 1997, 56-73; англ. пер.: V. N. Dubinin, "Asymptotics of the module of a degenerating condenser and some of their applications", J. Math. Sci. (N. Y.), 95:3 (1999), 2209-2220.

[115] В.Н.Дубинин, Л. В. Ковалев, "Приведенный модуль комплексной сферы”, Аналитическая теория чисел и теория функиий. 15, Зап. научн. сем. ПОМИ, 254, ПОМИ, СПб., 1998, 76-94; англ. пер.: V. N. Dubinin, L. V. Kovalev, "The reduced module of the complex sphere", J. Math. Sci. (N. Y.), 105:4 (2001), 2165-2179.

[116] D. Tischler, "Critical points and values of complex polynomials", J. Complexity, 5:4 (1989), 438-456.

[117] S. Smale, "The fundamental theorem of algebra and complexity theory", Bull. Amer. Math. Soc. (N.S.), 4:1 (1981), 1-36.

[118] A. F. Beardon, D. Minda, T. W. Ng, "Smale's mean value conjecture and the hyperbolic metric", Math. Ann., 322:4 (2002), 623-632.

[119] В.Н. Дубинин, "О покрытии вертикальных отрезков при конформном отображении", Матем. заметки, 28:1 (1980), 25-32; англ. пер.: V.N. Dubinin, "Coverings of vertical segments under a conformal mapping", Math. Notes, 28:1 (1980), 476-480.

[120] A. Eremenko, "A Markov-type inequality for arbitrary plane continua", Proc. Amer. Math. Soc., 135:5 (2007), 1505-1510.

[121] Н. С. Ландкоф, Основы современной теории потенииала, Наука, М., 1966, 515 с.

[122] G. Pólya, "Beitrag zur Verallgemeinerung des Verzerrungssatzes auf mehrfach Zusammenhängende Gebiete", S. B. Preuss. Akad. Wiss., 1928, 228-232.

[123] Г. В. Кузьмина, "Методы геометрической теории функций. I", Алгебра и анализ, 9:3 (1997), 41-103; англ. пер.: G. V. Kuz'mina, "Methods of geometric function theory. I", St. Petersburg Math. J., 9:3 (1998), 455-507; Г. В. Кузьмина, "Методы геометрической теории функций. II", Алгебра и анализ, 9:5 (1997), 1-50; англ. пер.: G. V. Kuz'mina, "Methods of geometric function theory. II", St. Petersburg Math. J., 9:5 (1998), 889-930.

[124] I. Schur, "Über die Verteilung der Wurzeln bei gewissen algebraischen Gleichungen mit ganzzahligen Koeffizienten", Math. Z., 1:4 (1918), 377-402.

В. Н. Дубинин (V. N. Dubinin)

Дальневосточный федеральный университет,

Владивосток

E-mail: dubinin@iam.dvo.ru
Поступила в редакцию 12.09 .2011 